University of Louisville

ThinkIR: The University of Louisville's Institutional Repository

Electronic Theses and Dissertations

8-2017

\title{
Custom software for the 3D printing of patient specific plate bending templates in pelvic fracture repair.
}

Gordon B Lents

University of Louisville

Follow this and additional works at: https://ir.library.louisville.edu/etd

Part of the Orthopedics Commons, and the Other Biomedical Engineering and Bioengineering Commons

\section{Recommended Citation}

Lents, Gordon B, "Custom software for the 3D printing of patient specific plate bending templates in pelvic fracture repair." (2017). Electronic Theses and Dissertations. Paper 2812.

https://doi.org/10.18297/etd/2812

This Master's Thesis is brought to you for free and open access by ThinkIR: The University of Louisville's Institutional Repository. It has been accepted for inclusion in Electronic Theses and Dissertations by an authorized administrator of ThinkIR: The University of Louisville's Institutional Repository. This title appears here courtesy of the author, who has retained all other copyrights. For more information, please contact thinkir@louisville.edu. 


\title{
CUSTOM SOFTWARE FOR THE 3D PRINTING OF PATIENT SPECIFIC PLATE BENDING TEMPLATES IN PELVIC FRACTURE REPAIR
}

\author{
By \\ Gordon Lents \\ B.S. University of Louisville, 2016

\begin{abstract}
A Thesis
Submitted to the Faculty of the University of Louisville J.B. Speed School of Engineering

As Partial Fulfillment of the Requirements

For the Professional Degree
\end{abstract} \\ MASTER OF ENGINEERING \\ Department of Bioengineering
}

August 2017 
CUSTOM SOFTWARE FOR THE 3D PRINTING OF PATIENT SPECIFIC

PLATE BENDING TEMPLATES IN PELVIC FRACTURE REPAIR

Submitted by:

Gordon Lents

A Thesis Approved On

$\overline{(\text { Date })}$

By the Following Reading and Examination Committee:

Michael Voor, Thesis Director

\begin{tabular}{c}
\hline Ayman S. El-Baz \\
\hline Kunal Kate \\
Brandi Hartley
\end{tabular}




\section{ACKNOWLEDGMENTS}

I would like to thank my mother, father, and sister for their constant encouragement and support and for continuously being role models throughout my life. I would like to thank my thesis mentor, Dr. Michael Voor, for his constant support and feedback throughout the entire project and Dr. Brandi Hartley for providing constant clinical input on the project as well as further insight into the field of orthopedic surgery. I would also like to thank Dr. Ayman El-Baz and Dr. Kunal Kate for taking the time to serve on my committee and for their contributions and oversight on this project. Finally, I would like to thank Dr. Gerald Grant for generously allowing me access to his laboratory which played an essential role in making this project possible. 


\section{ABSTRACT}

The purpose of this work is to reduce the operative time and blood loss incurred during open reduction and internal fixation (ORIF) of traumatic pelvic injuries through the creation of patient specific bending templates for reconstruction plates. These templates are 3D printed in a resin capable of being sterilized and taken into the operating room so that bending may be performed by the surgeon before the patient is opened or by another team member in parallel with the surgeon.

A novel software extension was created in 3D modeling software to allow a surgeon to individually position screws on a pelvic model to create a virtual plate. The software constrains the locations of placed screws so that the virtual plate is dimensionally identical to common reconstruction plates. The user is then able to export a bending template that includes the section of the pelvis the virtual plate was located on as well as screw location landmarks. The user can then flash sterilize the template and use it intraoperatively to obtain a plate that is accurately bent to the patient's anatomy and the surgeon's specifications. 
We produced a bending template representative of the most complex plating location on the pelvis, the posterior wall. A surgeon then accurately bent reconstruction plate to match the bending template, proving that the software produced a dimensionally accurate output. Other work has shown that the pre-bending of plates can shorten operative time, reduce blood loss, and allow for less invasive procedures. However, methods currently available for pre-bending patient specific plates involve the lengthy process of printing the patient's pelvis and then a lengthy sterilization process of the implant itself. Our method allows the template to be printed and processed in as little as 3 hours and sterilized by autoclave in less than 10 minutes.

Further work needs to be done to evaluate how the process works when used in a patient case, to statistically prove that our method reduces operative time and blood loss, and show that plates bent using our method are similar between all members of the surgical team. 


\section{TABLE OF CONTENTS}

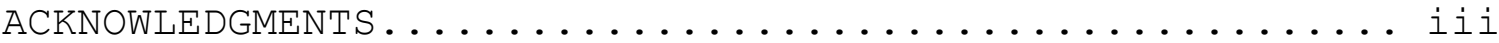

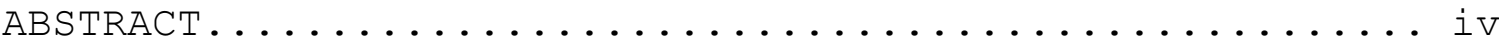

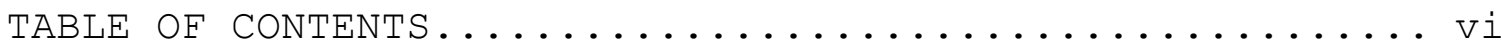

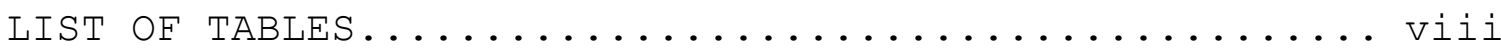

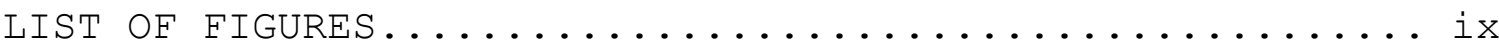

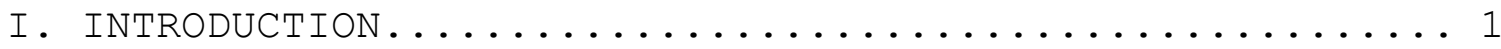

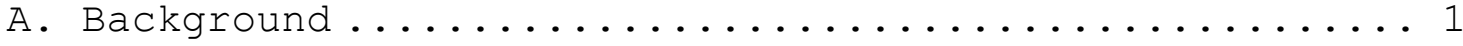

B. Review of 3D Printing for Plate Bending and Virtual

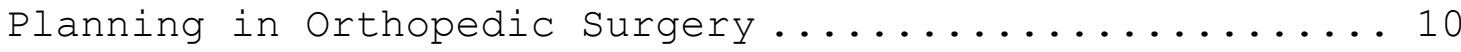

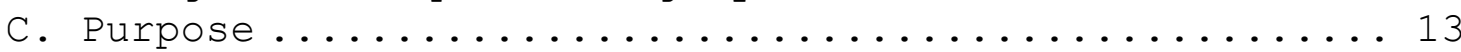

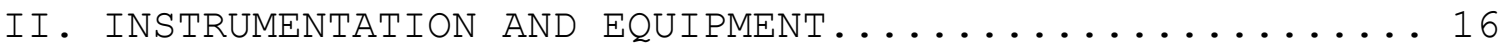

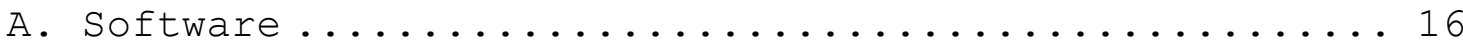

B. Formlabs Printer and Post-Processing Equipment ..... 18

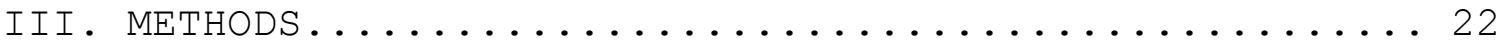

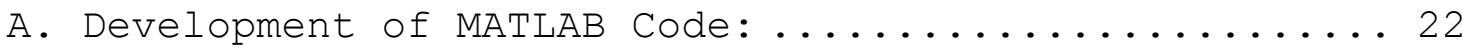

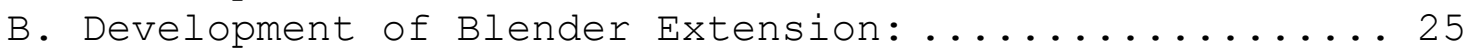

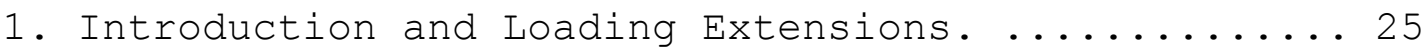

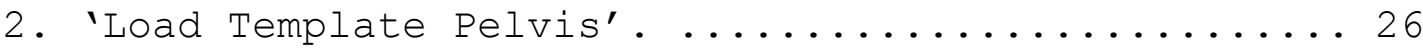

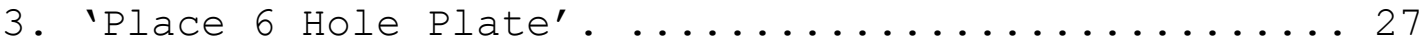

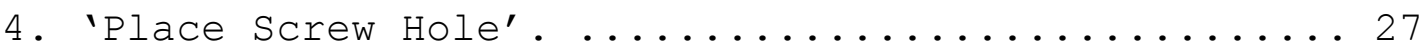

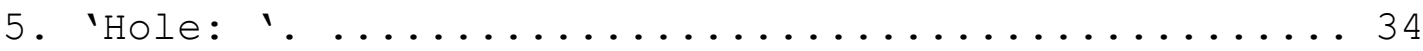

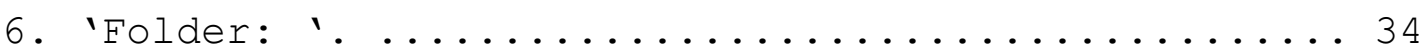

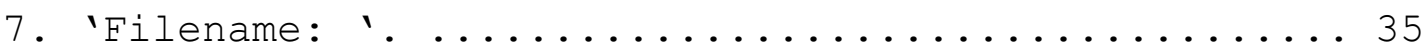

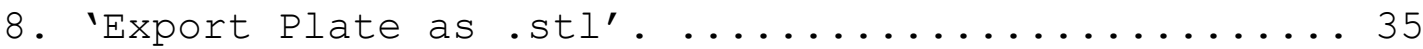

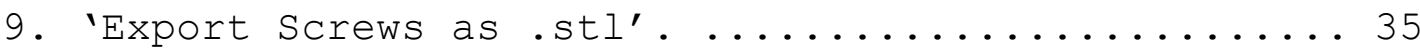

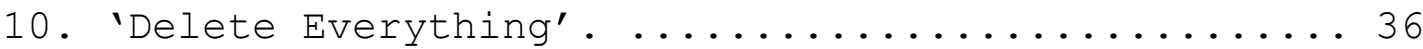

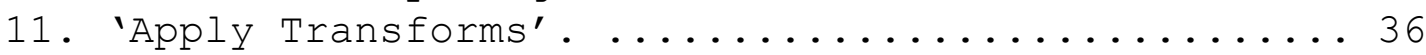

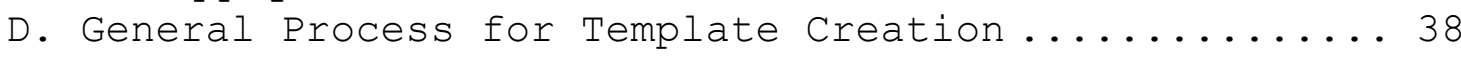

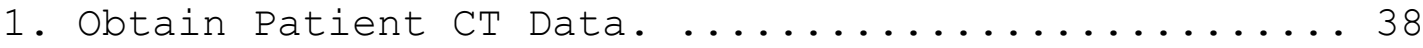

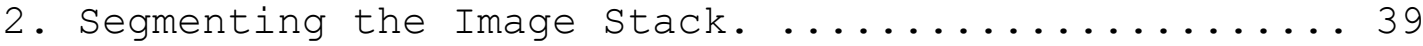

3. Edit Image Stack to Isolate Hemipelvis.......... 40

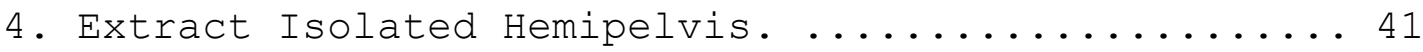

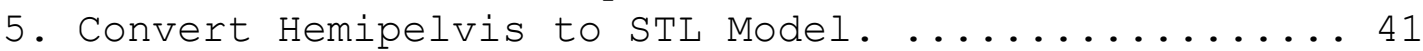

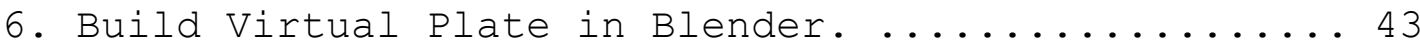

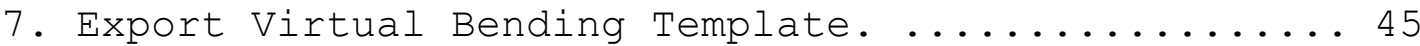

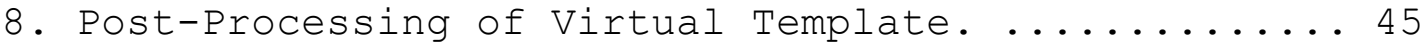

9. Preparing Template for Printing. .............. 46

10. Post-Processing of Physical Template...................... 47

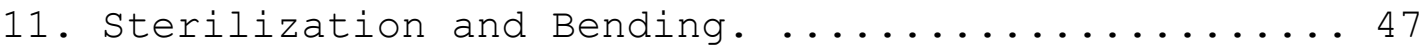

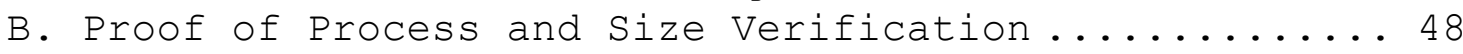

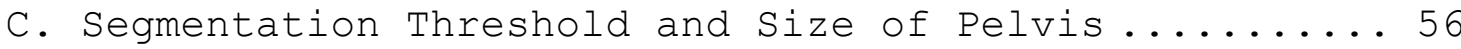

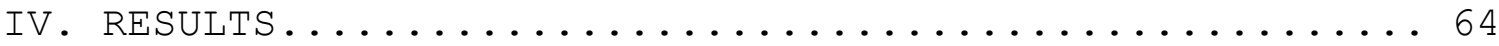




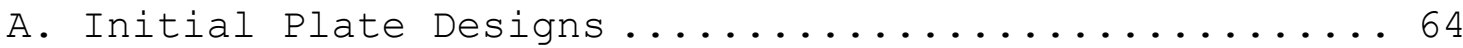

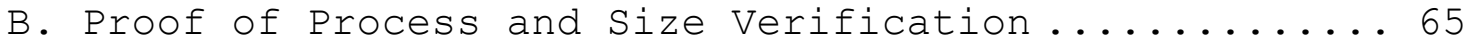

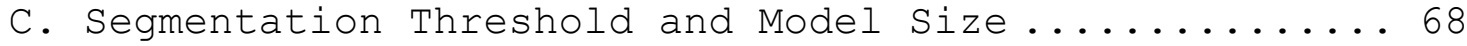

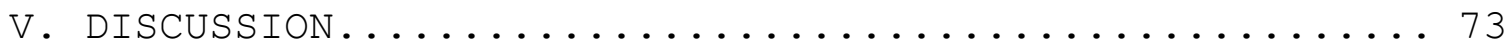

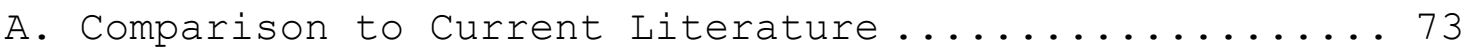

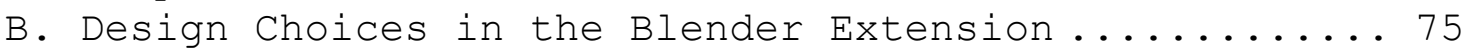

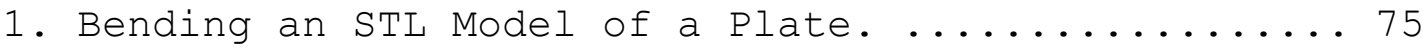

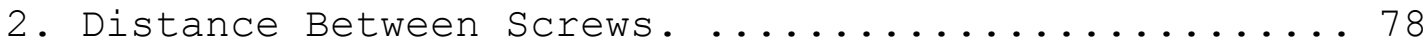

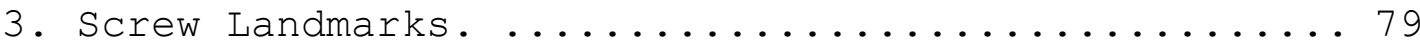

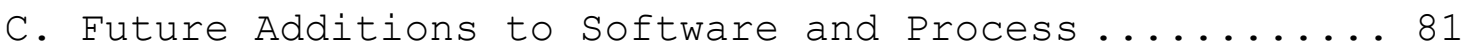

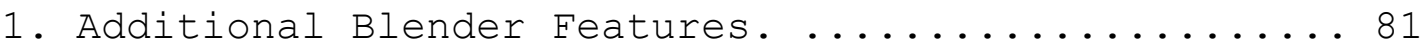

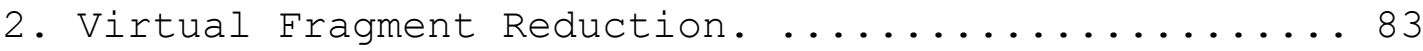

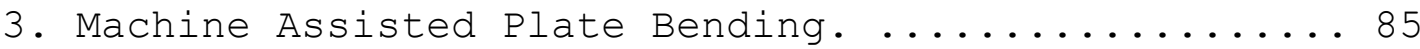

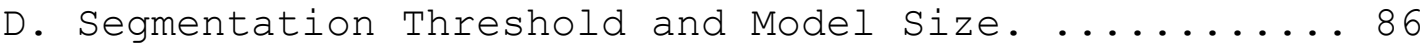

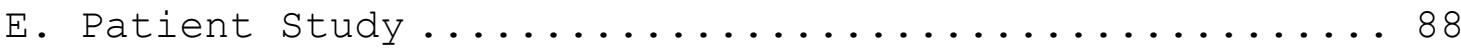

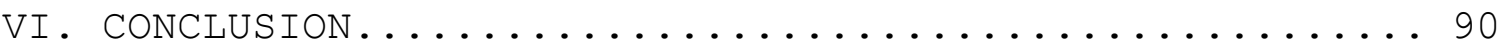

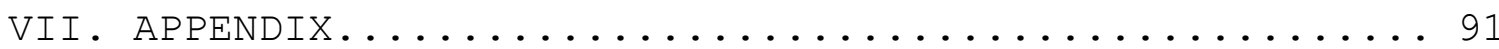

A. Text Output from MATLAB During Proof of Process ..... 91

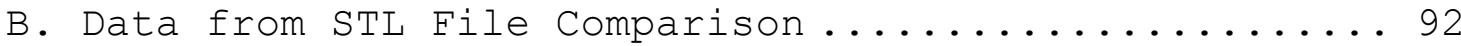

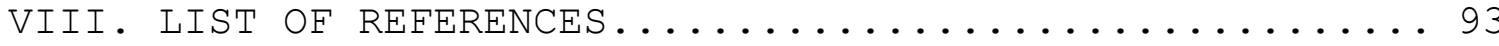

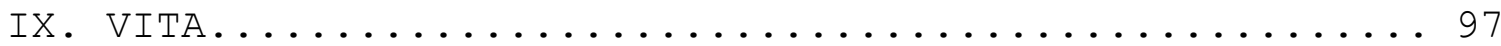




\section{LIST OF TABLES}

Table I - Dimensions of the Plates and Bases..........53

Table II - Parameters of the Cube Example.............63 Table III - Comparison of Designed and Measured Dimensions.

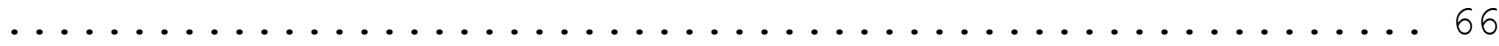
Table IV - Relevant Distance Values from Model Comparison 72 


\section{LIST OF FIGURES}

Page

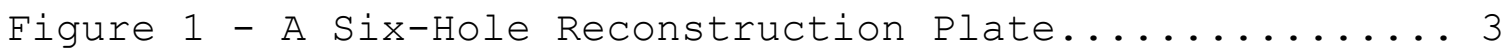

Figure 2 - Common Pelvis Plate Locations...............4

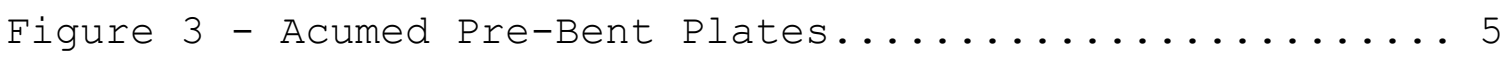

Figure 4 - Improperly and Properly Contoured Pelvic Wall

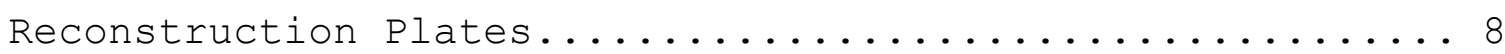

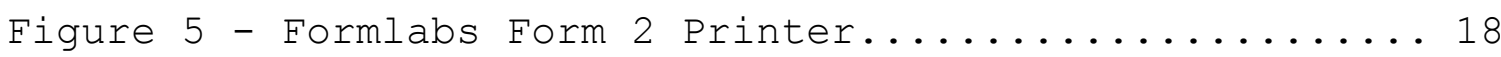

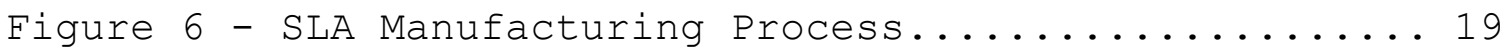

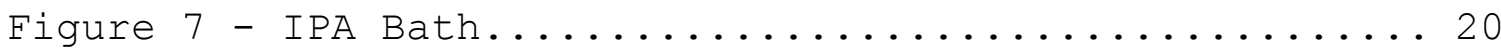

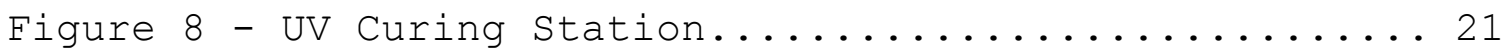

Figure 9 - Blender Extension Side Panel and Pelvis Model. 27

Figure 10 - Comparing Software Constraint Points to

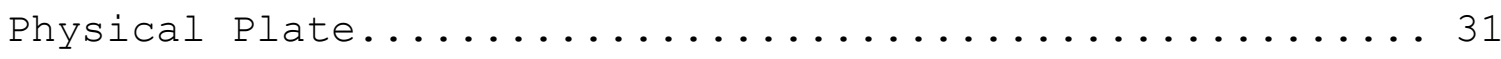

Figure 11 - Rotating and Translating the Pelvis........ 37

Figure 12 - Unaligned Pelvis Creates Skewed Screw Hole... 37

Figure 13 - Flowchart for Designing a Bending Template... 38

Figure 14 - Surgeon Specified Locations of Plates....... 50

Figure 15 - Surgeon Specified Posterior Wall Plate...... 51

Figure 16 - Surgeon Specified Anterior Brim Plate....... 51

Figure 17 - Pre-Processed Bending Templates with

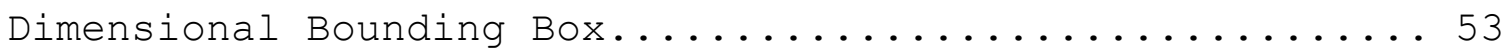

Figure 18 - Post-Processed Posterior Wall Virtual Template.

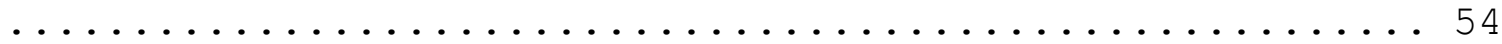

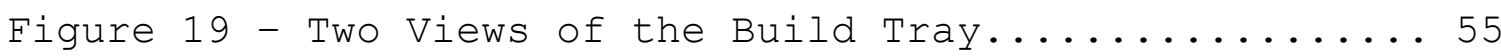

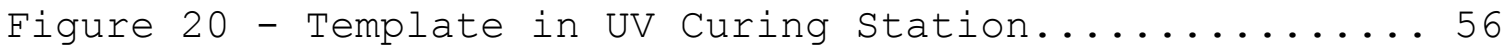

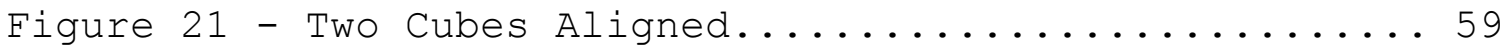

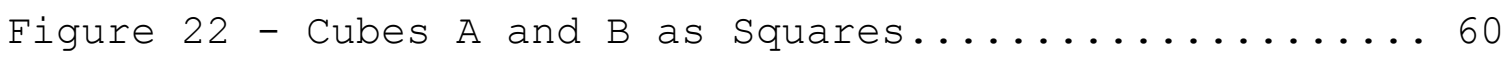

Figure 23 - Histogram of the Distances Between Points... 62

Figure 24 - Montage of Distance Colormap.............62

Figure 25 - Several Initial Bending Templates......... 64

Figure 26 - A Reconstruction Plate Bent to an Initial

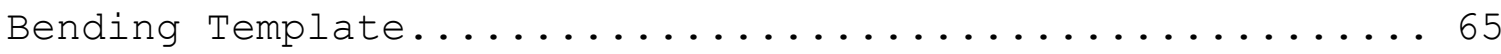

Figure 27 - Proof of Process Bending Template and Isotropic

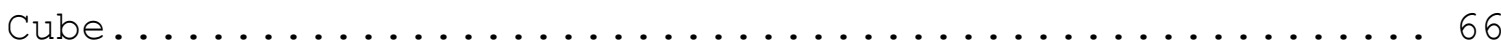

Figure 28 - Bending Template and Contoured Plate......6 67

Figure 29 - Locations of Large Distance Deviations......69

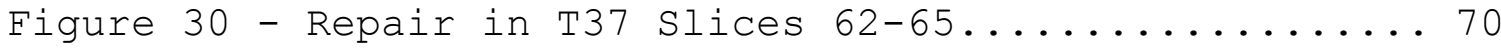

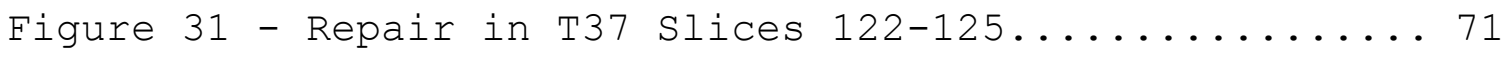

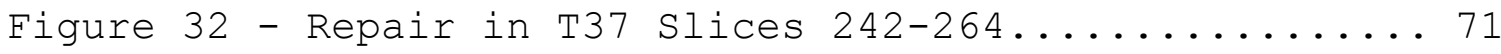

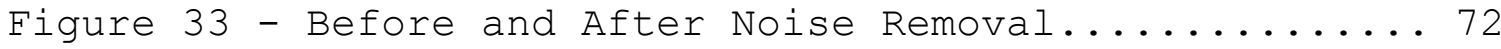

Figure 34 - Normal Bending of STL Model of Plate......76

Figure 35 - Glitches with Pre-Made STL Model of Plate... 77 
Figure 36 - Two Point Screw Dimensioning.............79

Figure 37 - Virtual Plate Overlaid on the Bending Template.

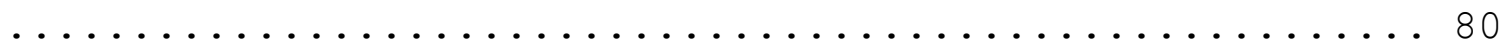

Figure 38 - Reducing Fracture Fragments in Blender.....8 84

Figure 39 - Maximum Deviations at Plate Locations.......87

Figure 40 - Maximum Deviations at Insignificant Locations.

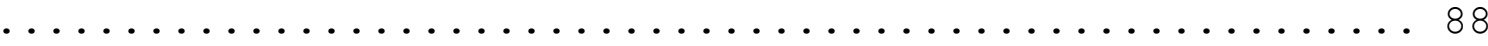




\section{INTRODUCTION}

\section{A. Background}

Treatment of any orthopedic injury begins with initial evaluation of the injury. Plain film x-rays are used as the initial evaluation in almost every injury but computed tomography (CT) scans provide a more accurate diagnosis [1]-[3] and are often used in more serious injuries. Many orthopedic injuries are not polytrauma but the most traumatic injuries can result in excessive internal bleeding, neurological damage, urogenital issues or other injuries that must first be dealt with before the orthopedic injury can be treated [4]-[6]. After the patient is medically stable, they can receive definitive treatment of their orthopedic injuries. Orthopedic treatment consists of reduction and fixation of the fractured bone fragments. Reduction is the process of moving the fractured pieces of bone back into their original anatomic locations and can be performed using non-surgical or surgical methods. The nonsurgical methods are called closed reductions and are preferred for minor injuries. Surgical, or open, reduction is performed in an operating room with the patient under 
anesthesia and is necessary when a complicated fractured has been sustained. Fixation is the process of ensuring that the bone fragments do not move from their reduced positions while the bones heal and is generally classified as either conservative, external, or internal. An example of conservative fixation is a plaster cast or a brace and is usually used to treat simple fractures. External fixation is a metal construct that consists of pins inserted through the skin and secured in bone and bars and clamps that reside outside of the patient's body to hold the pins in the proper location and orientation [7]. Patients with severe injuries will often need their fracture initially stabilized but will not be able to receive a definitive treatment until more serious injuries are resolved. External fixation allows for temporary fixation of a fracture while still allowing access to the surrounding soft tissue. Internal fixation is the current standard of care for many orthopedic injuries as it allows for quicker treatment and rehabilitation of the patient. Internal fixation is performed using K-wire, a thin smooth metal pin, screws, and reconstruction plates to hold fracture fragments in place.

Orthopedic injuries to the pelvis are often caused by high energy events such as motor vehicle accidents, 
being struck by a vehicle, or falling [5]. Pelvic fractures are often polytrauma and most patients receive both plain film X-rays and CT scans upon their arrival to the emergency department (ED). The definitive treatment for many pelvic injuries is open reduction and internal fixation (ORIF). While some injuries can be treated using only screws, the use of reconstruction plates is common and recommended for many pelvic ORIF procedures either because it is the only way to properly secure the fragments or because it offers increased stability and strength to the pelvis while it heals [8]. Reconstruction plates are made of either titanium or steel and generally consist of a straight piece of metal with holes that allow it to be secured to the bone using screws. Some plates, called locking plates, have threads manufactured into holes the screws pass through and allow the screws to lock into the bone and the plate in place. Non-locking plates rely solely on compression to keep the plate in the proper location.

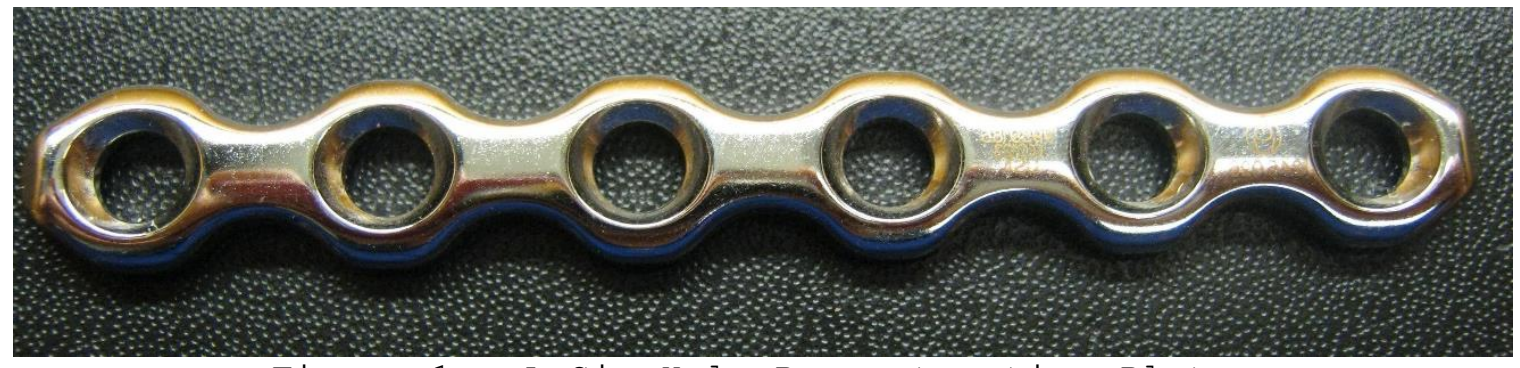

Figure 1 - A Six-Hole Reconstruction Plate. 
In many ORIF procedures, such as long bone repair, these reconstruction plates do not require any further modification to properly hold the fragments in place. The pelvis however, has a unique anatomy and often requires that plates be bent to maintain the correct position of the bone fragments. Common pelvic locations for reconstruction plates include the pubic symphysis, iliac wing, anterior brim, and acetabulum [4], [9]-[12].
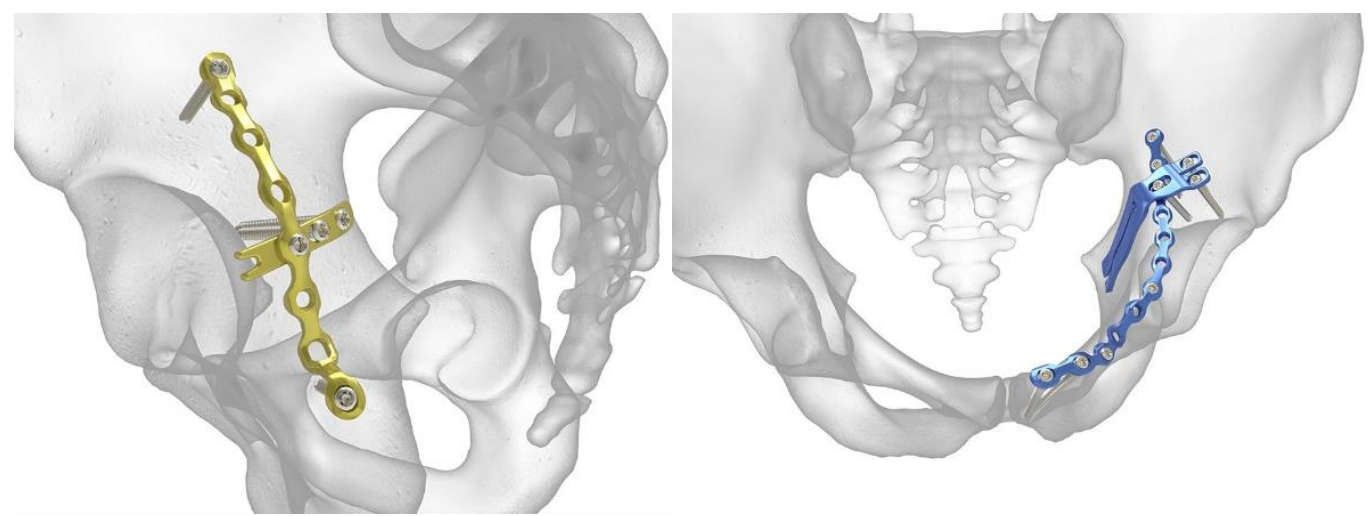

Figure 2 - Common Pelvis Plate Locations. Left image is a Posterior Wall Plate and the right image is an Anterior Brim Plate [13].

Some orthopedic sets have used anatomic data from large populations to create pre-bent plates for locations like the anterior brim and posterior column[13]-[16]. 

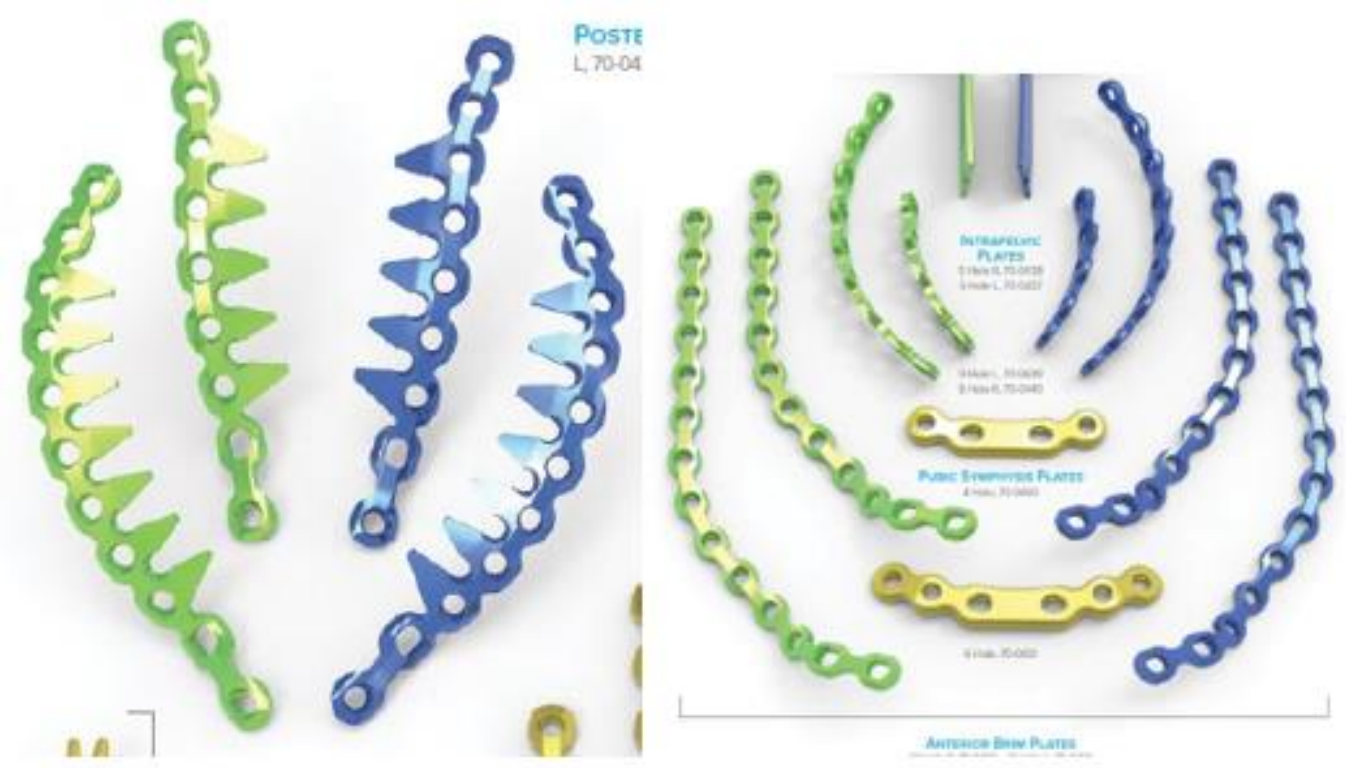

Figure 3 - Acumed Pre-Bent Plates. The left-hand image is of Acumed Posterior Wall plates and the right-hand image is of Acumed intrapelvic, anterior brim, and pubic symphysis plates [13].

However, the patient anatomy and fracture pattern is different for every case and these plates will often require further bending to properly contour to the pelvis and provide the necessary fixation. Additionally, it has been shown that since these pre-bent plates are often designed using dimensions of a single demographic, the fit of the plate and subsequent quality of the fixation varies across patient populations[17]. To assist in the bending process, some orthopedic sets contain additional malleable aluminum plates. This allows the surgeon to contour the aluminum plate to the reduced fragments and then bend the titanium reconstruction plate to match their aluminum 
plate. Regardless of the type of plate used in the surgery, all reconstruction plates will still require further fine tuning to match the exact anatomy of the patient that can only be achieved after the patient has been surgically opened and the fracture has been fully reduced. This requires the surgeon to expose the fracture site and open several other windows in the patient to assist with visibility and reduction during the surgery [18]. The plate bending process is not well described in orthopedic textbooks or literature and this knowledge is most often gained by surgeons through observation or practice within the operating room (Correspondence with Brandi Hartley M.D., 2017). In 'Fractures of the Pelvis and Acetabulum' Tile, Helfet, and Kellam [6] state that posterior column fractures can be fixed

"with a well-contoured plate that crosses the main fracture line of the posterior column." (p.636)

Similarly, when treating an anterior wall fracture,

"it is more effective to pre-contour a pelvic reconstruction plate and slide it under the vessels...centered along the pelvic brim" (p.639)

and when repairing a fracture of the anterior column

"definitive fixation is provided by the application of a pelvic reconstruction plate. The plate must be accurately contoured to the pelvic brim." (p.640) 
Gardner and Henley expanded upon descriptions of contouring methods in their book, 'Fracture Surgery' [18]. The authors provide the following instruction to aid in contouring a pubic symphysis plate,

"Place a slight pre-bend in the center and again just medial to both peripheral holes" (p.129).

When describing posterior column repair, Gardner and Henley simply state that an,

"under-contoured 3.5 reconstruction plate is usually sufficient for the application" (p.152),

and while describing the contouring of an intrapelvic plate they state,

"It should have a slight under contoured bend to accommodate the quadrilateral surface and should be twisted...to allow easier screw insertion into the superior ramus." (p.163)

'Surgical Treatment of Orthopedic Trauma' by Stannard, Schmidt, and Kregor provides the most detail on how specific reconstruction plates should be contoured [19]. When describing how to contour pubic symphysis plates the authors state,

"The surgeon bends the superior plate down 15 degrees before the last hole on each side of the plate where the pubis bone connects with the rami." (p.453)

For plates placed on the posterior wall, the authors do not describe how to correctly bend the plate but instead point out the following three common errors in plate bending: 
"Over-contouring of the plate so that the superior and inferior aspects of the plate contact the bone, but the plate over the midsubstance is not sufficiently buttressing the posterior wall" (p.490),

"not bringing the plate peripheral enough on the acetabulum so the posterior wall is not adequately buttressed" (p.490)

and,

"not bending the plate 'on the flat' so that the plate instead goes straight up the posterior column rather than curving more anteriorly above the acetabulum." (p.490)

The authors do not describe any contouring for anterior brim plates but it is known that additional contouring of pre-bent reconstruction plates is often necessary to bend them around the pubic ramus (Correspondence with Brandi Hartley M.D., 2017), [18]

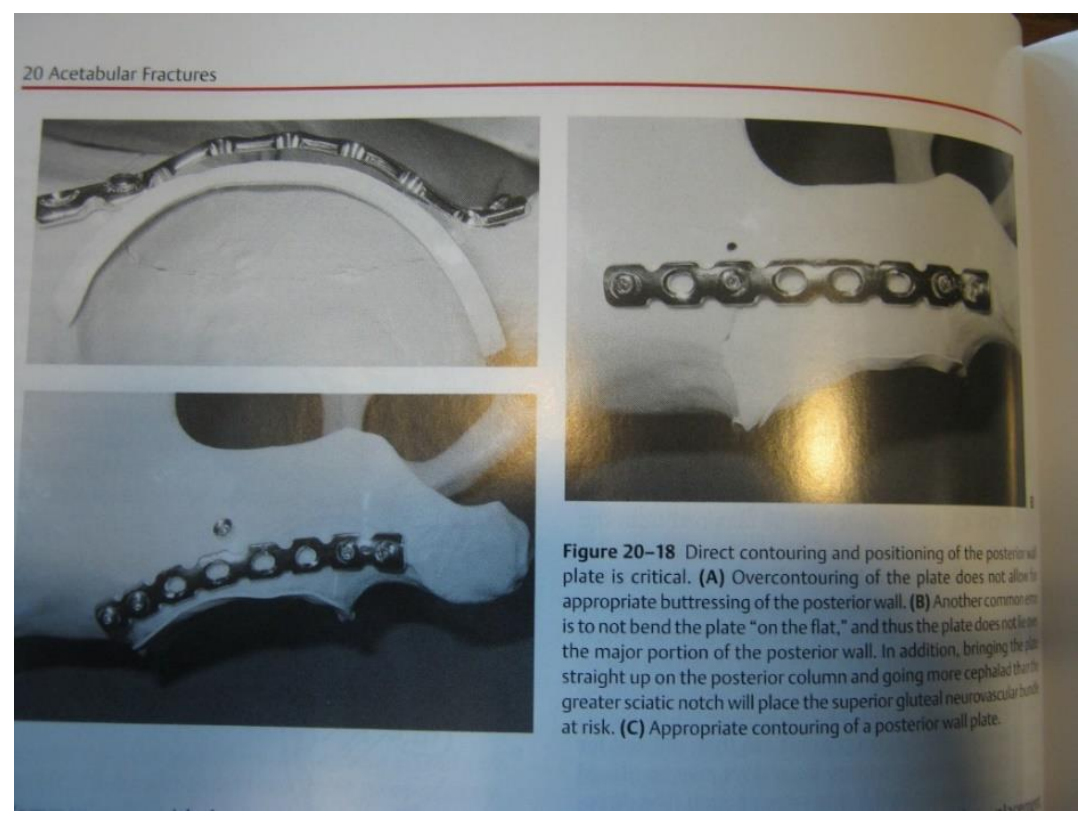

Figure 4 - Improperly and Properly Contoured Pelvic Wall Reconstruction Plates. [19] The top figures show improperly bent posterior wall plates. The bottom left image shows the appropriate contouring of the plate. 
We can see that the use of contoured plates is heavily described in orthopedic textbooks but descriptions of the methods needed to achieve these accurately contoured plates are rarely included. Most often, plate bending knowledge and techniques are passed along in the operating room during training making the quality of and the time needed to bend a reconstruction plate a function of the surgeon's experience. This potentially leaves a gap in the knowledge of surgeons who either do not see a high volume of orthopedic trauma or who are still in training. It also prevents other surgical team members such as technicians, nurses, or residents, from taking over what should be a relatively simple task.

The quality of the reduction of a pelvic fracture is a key determinant in how well the injury will heal which makes the accuracy of the reconstruction plate essential to the patient having a good recovery. Not achieving a proper reduction or not providing an adequate fixation to hold the reduction will result in a variety of complications such as osteoarthritis as well as osteoarthritis, gait problems, limb length discrepancies, urinary tract problems, and pain $[6],[20]$. 
The goal of all ORIF procedures is to provide functionality back to the patient as quickly as possible and can be accomplished through proper reduction and fixation. However, greater blood loss during a procedure correlates to poor clinical outcomes and increased time in the operating room increases the risk to the patient and the cost of the procedure. This makes evident the need for a solution that can provide high quality fixation, reduce operative time and blood loss, and make the plate bending process more accessible to less experienced surgeons and other surgical team members.

B. Review of 3D Printing for Plate Bending and Virtual Planning in Orthopedic Surgery

Methods for bending orthopedic plates to conform to a specific patient's anatomy have been described for the jaw and the pelvis. Some of these methods simply expand upon the idea of using a saw bone for surgical visualization and some methods advocate for or demonstrate the 3D printing a completely patient specific reconstruction plate. The most advanced and practical methods currently available combine a virtual planning environment that allow the surgeon to plan the exact 
location of plates and screws and provide a physical output to assist the surgeon with the procedure.

3D printing as a method of surgical planning is becoming more common place in orthopedic surgery and is being used to print patient models [21]. This approach was used in a 2015 study to view the relative displacement of fragments before surgery [22]. Another study used similar methods, but the authors autoclaved their patient specific pelvic models to assist with bending their plates and visualizing fracture fragments while in the operating room. In a 2002 study, the authors used a printed model of their patients pelvis to contour a reconstruction plate and create a screw guide [23]. In a 2014 study, patient specific pelvic models were used to determine proper reconstruction plate size and screw lengths [24]. These models were also sterilized and taken into the operating room to give the surgeon the ability to examine the printed pelvis and fracture pattern as they repaired it or contour the plate to the surface of the pelvic model before implanting it in the patient. 3D Printed models are also common in oral and maxi-facial reconstruction. Multiple studies have created virtual, bent plates and compared their accuracy to that of a hand bent plate [25], [26]. Other studies used virtual methods to model the repair of a 
mandible and then printed the model to assist in the bending of their plate [27], [28].

Beyond the simpler methods described above, some authors have used the rise in rapid prototyping technology to create completely patient specific reconstruction plates and 3D print them in titanium using selective laser melting (SLM) or Electron Beam Melting (EBM) [29]. In addition to improved patient fit, researchers have shown increased strength [30], [31] and improved biocompatibility [32] are also possible with additive manufactured titanium implants. Despite these benefits, large regulatory barriers exist that make customized reconstruction plates rare and impractical in the United States.

Surgical planning software can combine patient specific details and avoid many of the regulatory hurdles that advanced additive manufacturing technologies face. A surgical planning system described in 2012 used data of the patient's intact anatomy as a base that the surgeon could draw their desire reconstruction plate onto [33]. They then used virtual reality (VR) equipment to project the virtual plate on-screen to guide the surgeon in bending an aluminum plate. The contoured aluminum plate was then sterilized, taken into the operating room, and used as a template to bend the titanium reconstruction plate. In 2007, Cimerman 
and Kristan created a software tool to assist in surgical planning [34]. Within the software, the user could segment and separate out all bone fragments as individual pieces, reduce the fractures, and add plates and screws to simulate fixation. Most recently in 2017, Chen used CT scan data and MIMICS to create screw guides and bending templates [35]. They then 3D printed their guide and template and demonstrated their use on cadaver pelvises. Using the screw guide, the authors first placed k-wire pins. They then removed the guide, bent a plate using their template, and placed the plate onto the pelvis. Finally, they removed the pins and replaced them with screws to hold the plate in place.

C. Purpose

This thesis will describe a method that allows the surgeon full flexibility within a novel software extension to precisely position a virtual plate to create an accurate bending template for a reconstruction plate. This anatomic bending template is 3D printed in a material that allows it to be sterilized and brought into the operating room. This removes the time delays associated with other methods that involve printing an entire hemipelvis or re-sterilizing the 
orthopedic implant. Finally, this method delivers a prebent reconstruction plate that produces identical, if not better, results than that which could be achieved through contouring the reconstruction plate on a saw bone or a 3D printed model of a patient's pelvis through the addition of screw landmarks on the template being printed. These landmarks provide tactile and visual confirmation to the user that the plate has been properly bent and allows for other members of the surgical team, such as technicians, nurses, or residents, to produce the same results as the surgeon that designed the plate. Other authors have proposed anatomically contoured and pre-bent plates as allowing the fixation of fractures to be done using less invasive methods than the traditional approaches [33], [36]. The current standard of care involves direct exposure of the fracture site to allow the surgeon to bend the plate to match the patient's anatomy and fracture pattern. Using our proposed method, the plate could be fully contoured before the patient is opened which will reduce the time needed for surgery, the blood lost by the patient, and could reduce the size of incision needed as well as remove the need for direct exposure of the fracture site. Overall, this system will allow for shorter operative times and reduced blood loss by the patient with the potential to 
allow for a less invasive surgery with better clinical outcomes. 


\section{INSTRUMENTATION AND EQUIPMENT}

A. Software

The following six software packages are used to create a physical bending template from DICOM CT images: Solidworks (Solidworks 2016, Solidworks Corp., Waltham, MA, 2016), ImageJ [37], MATLAB (MATLAB 9.2, The MathWorks Inc., Natick, MA, 2017), Slicer 4.7.0 [38], Blender (Blender 2.78, Blender Foundation, Blender Institute, Amsterdam), Autodesk Meshmixer (Meshmixer 3.2.37, Autodesk Inc., San Rafael, CA, 2017), and Preform (Preform 2.11.3, Formlabs Inc., Somerville, MA, 2016). Additionally, CloudCompare (CloudCompare 2.9, 2017) was used for STL file comparison. ImageJ is used to import DICOM files, process them, and export them as an image stack in a TIFF format. TIFF is a useful file format for working with medical imaging data because it allows all slices, or individual images, of the data set to be included in a single file and makes handling the images much simpler. Image processing in ImageJ 
includes threshold segmentation, hole filling, erosion and dilation as necessary to remove small fragments, and manual separation of the intact hemipelvis from the sacrum and other structures. MATLAB is used to calculate the amount of independently connected objects in a 3D image stack and separately save them. Slicer is used to rebuild 3D image stacks, smooth them, and export them as stereolithography (STL) files. STL files are 3D objects that have had their surfaces rebuilt using triangles and are the most common file format used in additive manufacturing. The STL reconstruction is then imported into Blender where a novel software extension has been designed to allow the virtual placement of a reconstruction plate. Inputs obtained during the virtual plate building process are then used to create a bending template that can be 3D printed. After the creation of the virtual bending template, the template is exported from Blender as an STL file and final processing is done within Meshmixer. Meshmixer allows for editing and preparation of STL files before they are 3D printed. Within Meshmixer, the virtual template model can be finalized and checked for errors to ensure it will print successfully. After final processing of the bending template within Meshmixer, the file is imported into Preform. Preform is the proprietary software utilized with Formlabs printers to 
prepare an object for printing. Preform is used to customize the settings that the 3D printer will use such as resolution, object orientation, and support structures.

\section{B. Formlabs Printer and Post-Processing Equipment}

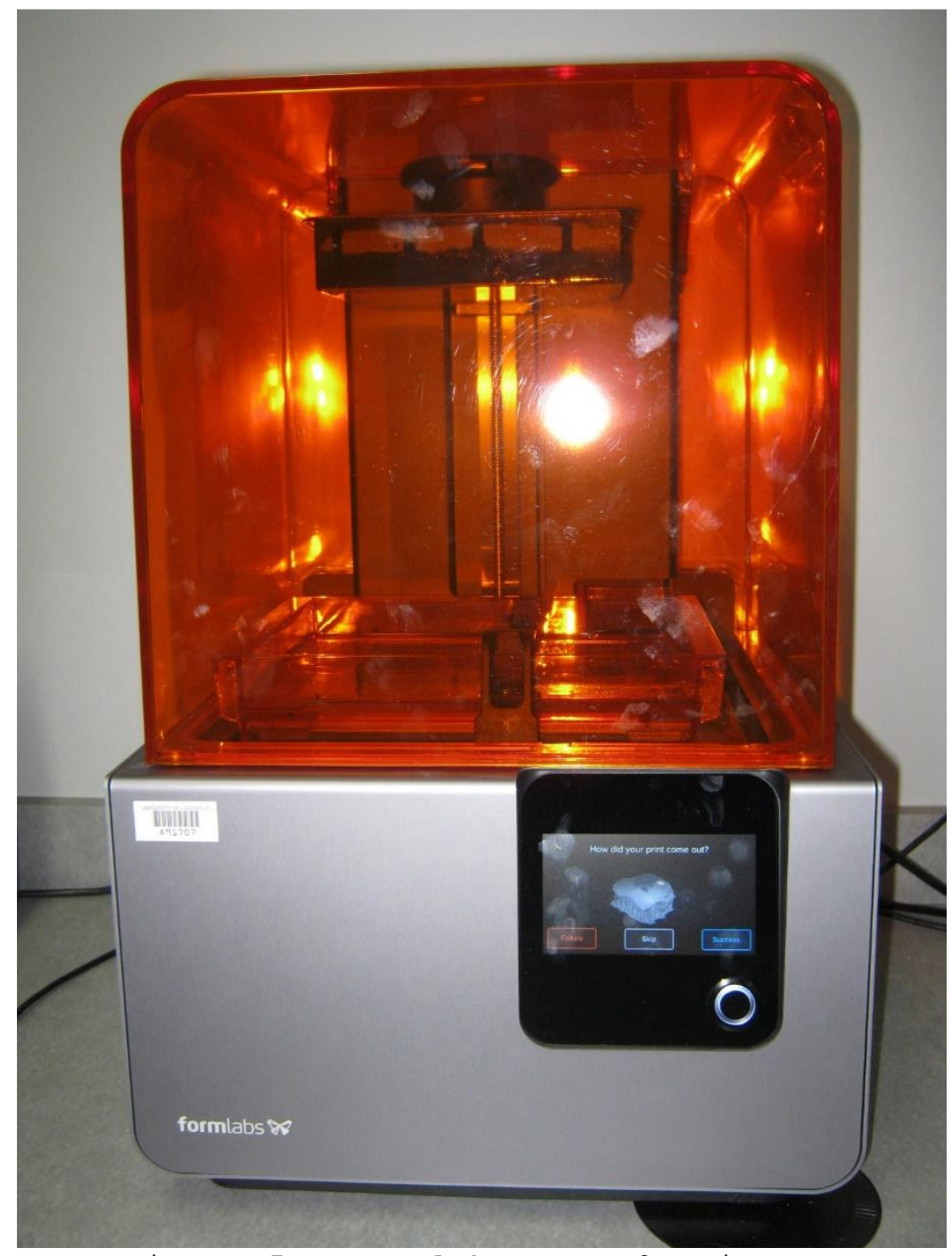

Figure 5 - Formlabs Form 2 Printer.

A Formlabs, Form 2 printer was used for all templates

printed for this work. The fundamental components of the 
Form 2 are the UV shield, the build platform, the resin tray, and the resin cartridge. The build platform is where the part is attached and can be removed from the printer. The resin tray contains a reservoir of resin that is used during manufacturing and is refilled from material in the resin cartridge. The printer can only manufacture in a single material per print but the cartridge can be switched to allow for different materials to be used for different prints. The Form 2 utilizes SLA printing technology to create solid objects in a layer by layer fashion. In this method, the build platform is lowered into the tank of resin and a UV laser cures the material that is needed to create that layer of the part. After each layer is complete, the platform raises slightly and the next layer is created. The Form 2 is capable of build parts with layers as small as $25 \mu \mathrm{m}$ and has a beam resolution of $140 \mu \mathrm{m}$ [39].

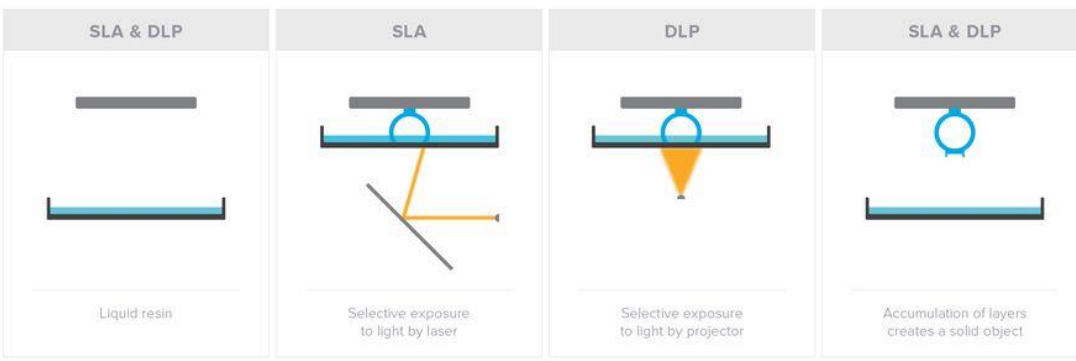

Figure 6 - SLA Manufacturing Process. This image compares and contrasts the manufacturing techniques of SLA and DLP, both of which involve light cured polymers [40]. 
The resin used in the Form 2 printer is made up of a (meth) acrylated monomers, (meth) acrylated oligomers, and photo initiators. This polymer solution remains a liquid until it is exposed to UV light which is why the UV shield must always be in place unless the parts are being removed. The use of the laser in the building process provides a majority of the energy needed to solidify the resin but final post-processing must be performed to create the highest quality part.

For post processing, a bath containing 90\% isopropyl alcohol (IPA) and UV curing station are necessary.

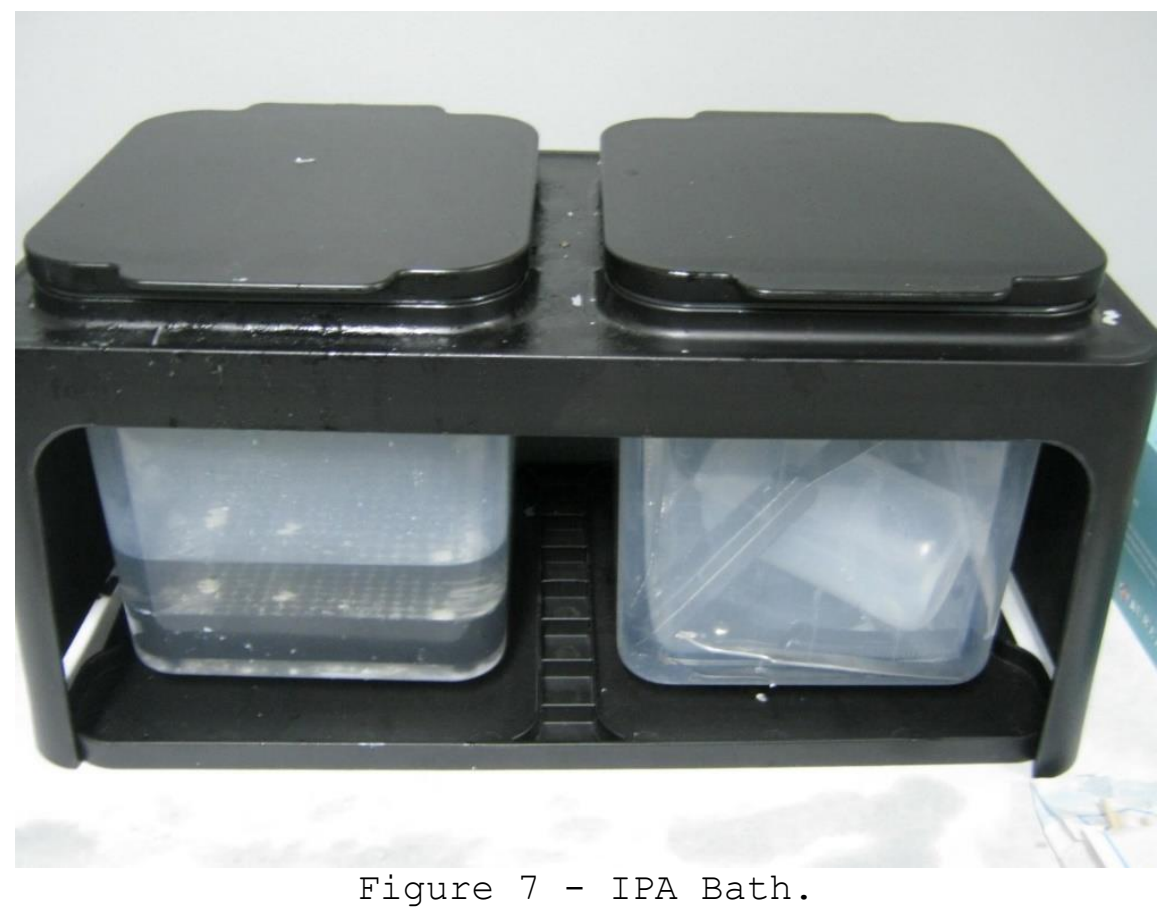




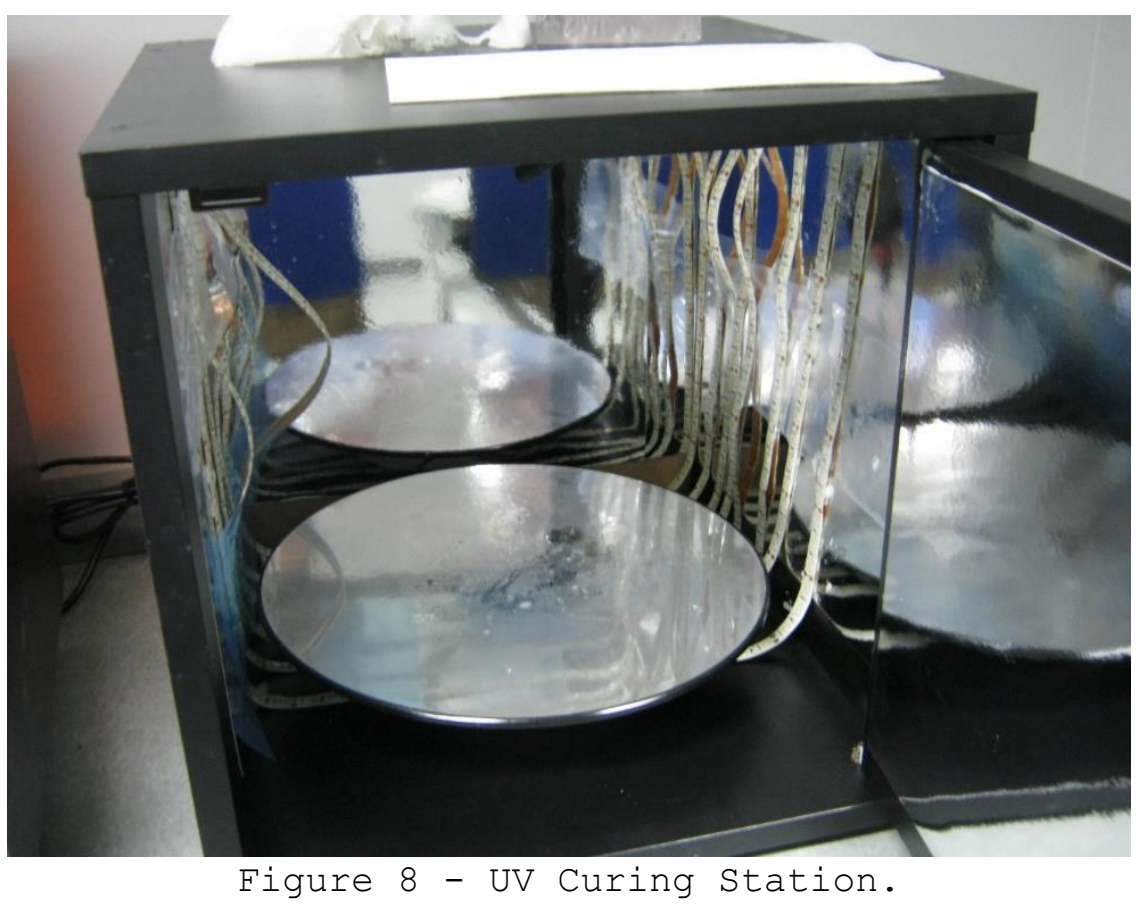

After being removed from the print platform, the user should immediately place the part into a bath containing fresh, 90\% isopropyl alcohol (IPA) and allow it to sit for 10-20 minutes. This process removes any residual, uncured resin that might remain on the part. After the IPA wash, the part should be transferred to a UV curing station with 108 Watt, 315-400 nm and 400-550 nm wavelength bulbs and allowed to cure for 10 minutes. Placing the part in the UV curing station ensures that all photopolymers making up the part have been fully cured and solidified. 


\section{METHODS}

A. Development of MATLAB Code:

MATLAB, or Matrix Laboratory, is a programming language and compiler that specializes in matrix operations and is commonly used for image processing tasks such as thresholding, segmentation, and other morphological processing. For this thesis, two additional MATLAB packages were utilized: The Image Processing Toolbox and the Parallel Processing Toolbox. The Image Processing Toolbox provides additional functions that are specifically tailored for editing medical images and image stacks. The parallel processing toolbox allows MATLAB to take advantage of multi-core central processing units (CPU) when performing computationally heavy tasks. Thresholding the original CT image within ImageJ will result in a binary image that only contains pixels labeled 0, completely black, or 255, completely white. The white pixels represent a unit of bone within two dimensions. In a three-

dimensional image stack, such as a CT scan, the units of 
bone are known as voxels and contain a height dimension in addition to length and width. To rebuild the intact pelvis from an image stack, the white voxels that make up the intact hemipelvis must be separated from the white voxels that represent other structures such as the sacrum, bone fragments, and miscellaneous noise. MATLAB includes a function, "bwconncomp", that detects connected pixels in a binary image or connected voxels in a stack of images. After initial thresholding and manual separation of bones within ImageJ, MATLAB is used to isolate the intact pelvis for further reconstruction.

Before the software is run, the user has the opportunity to enable and change the properties on several image modification parameters as well as set the level of connectivity to be used, the size threshold, and specify if they want the fragments saved. After being started, the MATLAB code begins by allowing the user to select the TIFF image stack that they wish to open. The function then performs an initial calculation to determine the number of fragments, or connected collections of voxels, that are present in the image. A fragment is defined by the software as voxels that are touching based a certain connectivity both within the image itself and within the images above and below the image in question. The connectivity is 
controlled by the user and can be changed before the software is run. The lowest level of three-dimensional connectivity is 6 where only directly adjacent voxels are considered connected and the highest is 26 where only the corners of two voxels need to be touching for them to be considered connected. The initial fragment check reports two values, the total number of connected items and the total number of connected items above a certain size threshold. The size threshold is set by the user and useful for eliminating noise and very small fragments that are not of interest from the fragment total. Next, if any image editing processes were enabled by the user before the program was started, these are executed. The editing done to the image stack in ImageJ is only an approximation so this further image processing is useful to see how something like an erosion changes the number of fragments in the image. Often, an erosion in MATLAB is needed to fully disconnect the intact hemipelvis and sacrum. After image processing, the software recounts the number of connected voxel groups based on the processed stack of images and provides a new value of the total number of fragments and the number of fragments above the size threshold. If the user elected to save the fragments before starting, the program will save each fragment as its own 
TIFF stack. The number of fragments saved will be equal to the number of fragments greater than the size threshold. The user can then open the fragment stacks to determine which file contains the bone of interest.

B. Development of Blender Extension:

1. Introduction and Loading Extensions. Blender is

a 3D modeling program that is commonly used in the development of assets for animations and games. The value of Blender in this thesis is that it allows for STL files to be imported and any model built within the software can be exported as an STL file. Additionally, Blender allows the user to develop extensions to the software by writing them in the programming language, Python (.py). For this thesis, a software extension was written that allows for the creation of customized bending templates.

Extensions in Blender can be installed by selecting "File" -> "User Preferences...", selecting the top tab labeled 'Add-ons' and selecting the 'Install New Addons...' button at the bottom of the panel. The user can then navigate to the location of the add-on saved as a '. $\mathrm{py}^{\prime}$ file. The add-on should then be present in the Addons list. The user can now select the box at the left of 
the addon's name to enable it and afterwards should restart the software.

2. 'Load Template Pelvis'. To begin, the user must import the STL file of the pelvis they want to use to design the plate. For the development of the extension, a female hemipelvis from the online 3D database Thingiverse was used [41]. If the user presses the 'Load Template Pelvis' button, a high quality, right hemipelvis will be imported into the software. After the pelvis is imported but before control is given back the user, the software will center the pelvic model back middle of the screen, properly rename the model, and apply the necessary transforms so that plate building can occur. 

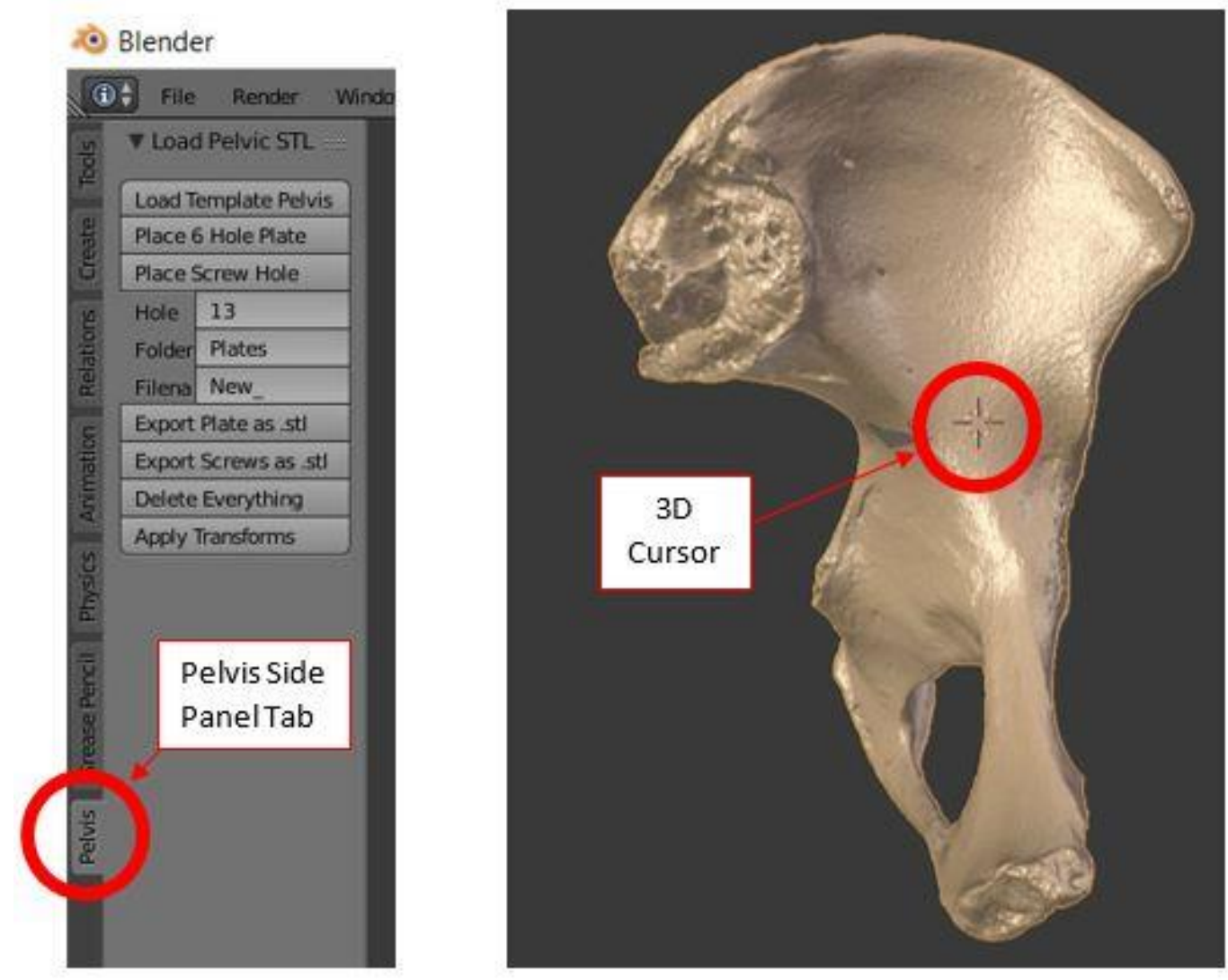

Figure 9 - Blender Extension Side Panel and Pelvis Model. This image points out the location of the side panel as well as identifying key items located in the Blender window.

\section{3. 'Place 6 Hole Plate'. This button will}

important a STL model of a six-hole plate that is

dimensionally similar to those currently on the market.

This function is not used in current methods of plate

bending and its function is discussed during the

discussion.

4. 'Place Screw Hole'. This button is the backbone

of the virtual plate creation software. After a pelvis is 
loaded and oriented, the user can left-click on the pelvis where the first screw will be located. The 3D cursor moves to that location and pressing the 'Place Screw Hole' button on the side panel places the first screw.

When the 3D cursor is first placed onto the pelvis, the software selects the nearest triangle making up the surface of pelvic model. The software then expands the selection of triangles outward several times to cover a similar area to that of overlying virtual plate. The central point and the average normal vector to the selection of triangles are calculated. An STL model representing the screw hole was created in Solidworks. It is 3mm thick, has an outer diameter of $10 \mathrm{~mm}$ and an inner diameter of $4.5 \mathrm{~mm}$ to mimic the dimensions of many Stryker reconstruction plates. When the screw hole is placed, this STL file is imported into the software, placed at the calculated center point, and rotated based on the average normal of the triangles below it. 'Shrinkwrap' is a constraint available within Blender to constraint one object to the surface of a target and a setting is available to offset the object a certain distance from the target's surface. The software then applies a 'shrinkwrap' constraint to the imported screw hole with an offset of $1.5 \mathrm{~mm}$. Since the total thickness of the imported screw hole 
model is 3mm, this constraint places the virtual plate exactly on the surface of the pelvis. After the screw hole is imported but before the software gives control back to the user, a $3.75 \mathrm{~mm}$ sphere is imported and a circle is created at the same central location of the screw hole. The sphere has the 'shrinkwrap' constraint applied with no offset distance and is a landmark showing where a screw would be placed on the real pelvis. The circle undergoes the same rotations as the screw hole and represents the distance at which the user should place their next screw hole. Since this virtual plate is intended to mimic a physical reconstruction plate that must eventually be bent, the software has several constraints to ensure the dimensions of the virtual plate will mimic the dimensions of the real plate as closely as possible. This circle is created with a user defined radius that corresponds to the distance between holes on the physical reconstruction plate. The default setting is for a plate with a distance of $13 \mathrm{~mm}$ between screw holes, making the radius of the circle $13 \mathrm{~mm}$.

$\frac{\text { Total Plate Length }(\mathrm{mm})}{\text { Number of Screw Holes }}=$ Distance Between Screw Holes 
The intention is for the user to place the next screw on the perimeter of this circle to avoid improper orientation or dimensioning of subsequent screw holes. The second screw hole is placed similarly to the first, by placing the 3D cursor at the desired location and clicking 'Place Screw Hole' on the side panel. During the placement of every screw after the first, three constraint points are created between the center points of the screws. The constraint points are placed to keep the virtual plate dimensionally accurate while allowing for bending along the surface of the pelvic model. The three constraint points coupled with the center point of each screw create five total points, or four segments, between each screw. 


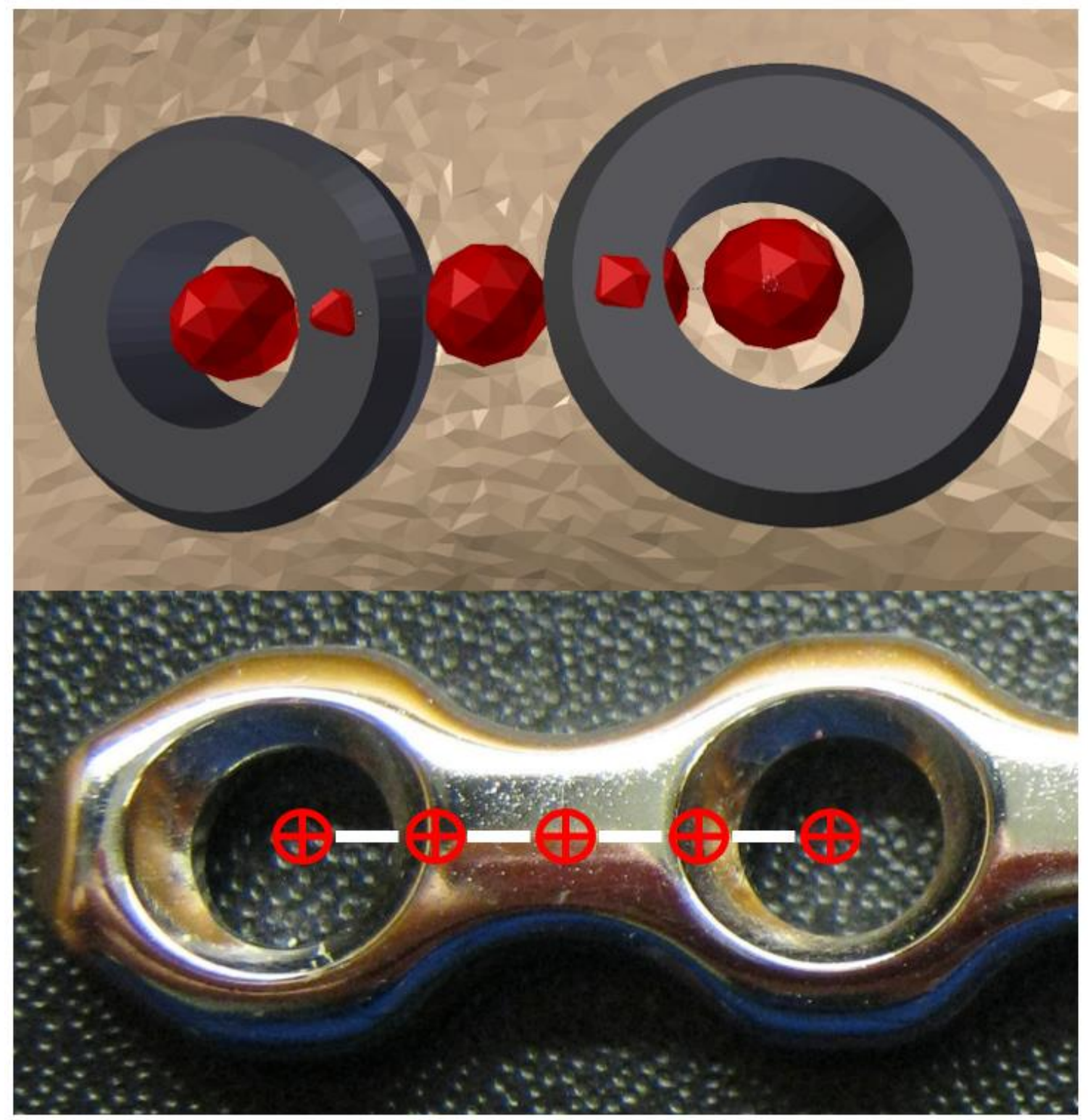

Figure 10 - Comparing Software Constraint Points to Physical

Plate. The top figure shows the constraint points in the software with red spheres to emphasize their location. The bottom photo shows where these constraint points would be located on an actual plate (red circles) and the 4 segments that are dimensionally controlled (while lines).

Each segment is locked to be the total spacing between

screw holes divided by four. \{picture of a real plate might help here with overlaid dots to show where the constraint 
points are; maybe a picture of the process in Blender as well\}.

$$
\frac{\text { Screw Distance in Plate }}{\text { Number of Segments }}=\text { Length of Segment }
$$

$$
\frac{13 \mathrm{~mm}}{4}=3.25 \mathrm{~mm}
$$

The creation of these points for a plate with hole spacing of $13 \mathrm{~mm}$ is as follows: After the second screw hole is placed and the $1.5 \mathrm{~mm}$ 'shrinkwrap' constraint is applied to it, a line is drawn between the first and second screw holes and the midpoint of that line is calculated. This midpoint is redefined as "Empty 2". Empty 2 has the 'shrinkwrap' constraint applied to it with an offset of 1.5mm from the surface of the pelvis. A new line is now created between the first screw hole and the partially constrained Empty 2 and the midpoint of that line is calculated. This midpoint is redefined as "Empty 1 " since it is the closest constraint point to the first screw hole. Empty 1 has the 'shrinkwrap' constraint applied with an offset of $1.5 \mathrm{~mm}$ from the pelvis' surface and a distance constraint of $3.25 \mathrm{~mm}$ applied between itself and the center of the first screw hole. Empty 2 now has a distance 
constraint of $3.25 \mathrm{~mm}$ applied between itself and Empty 1 . A third midpoint is calculated between the fully constrained Empty 2 and the initial location of the second screw hole. This midpoint is defined as "Empty 3" and is the closest constraint point to second screw hole. This midpoint has the same 'shrinkwrap' constraint applied as the other points and has a distance constraint of $3.25 \mathrm{~mm}$ applied between itself and Empty 2. Finally, the second screw hole location center point has a distance constraint of $3.25 \mathrm{~mm}$ applied between itself and Empty 3 and distance constraining between the screw holes is now finished. Using this method, the virtual plate can be placed over almost any surface while retaining a high level of dimensional accuracy between itself and the physical reconstruction plate. In the above description, the hole spacing was assumed to be $13 \mathrm{~mm}$ which is the default value. However, this value can be modified within the side panel to be made compatible with any reconstruction plate the user might have. Assuming the distance between the midpoint of final hole and the end of the plate is equal to half the distance between each screw hole ppicture of a plate to emphasize this\}, the distance between screw holes can be calculated as seen in Equation 2 . 
Subsequent screw holes can be placed until the user is satisfied with the size and location of their virtual plate. In some cases, the constraints placed on the screw holes will make a more complicated virtual plate than would be used in a surgery by fully conforming to the surface of the pelvis. In this case, the user can manually disable 'shrinkwrap' constraints on the screw hole and "Empty" points and move the plate as they see fit. As long as the distance constraints are kept in place, the virtual plate will still reflect the dimensions of the physical reconstruction plate.

5. 'Hole: ' This input box gives the user the option to change the dimensioning of the screw holes. This value controls the dimensioning of the segments in between each plate and the radius of the circle placed around the most recent screw hole. The default value is set to $13 \mathrm{~mm}$ between holes.

6. 'Folder: ' This input box gives the user the ability to change the folder any outputs will be placed in. The software extension has a preliminary file path set that the user can edit through simple manipulation of the code. 
7. 'Filename: ' This input box gives the user the ability to change the file name of any outputs from the software. This value should be changed for each new plate that is planned to be built.

8. 'Export Plate as .stl'. This button will save the virtual, aesthetic plate as a STL file and then reimport it back into the scene. This gives the user the option to build multiple virtual plates and templates while still having a visual representation of where their previously designed plate is located.

9. 'Export Screws as .stl'. When this button is pressed, several actions will take place within the software. First, the software will activate all vertex groups located on the pelvis model. Every time the user placed a screw hole, a single triangle face underneath the screw hole was selected. Beginning with the second screw the user placed, these single triangle faces were connected in a line and saved as a vertex group, or group of triangles. When the user is ready to export their template, all previously created vertex groups become selected and this selection region is grown to encompass the area under the virtual plate. This selection of triangles is then 
copied, separated from the surface of the pelvis, and made into a solid with the Blender modifier, Solidify. This solidified section is extruded down $4 \mathrm{~mm}$, all spheres representing screw locations are selected by the software, and the new solid surface and the spheres exported as a single STL file. The software then deletes all spheres and constraint points.

10. 'Delete Everything'. This function selects all on-screen items excluding the pelvis and deletes them. It is used when the user wishes to restart the plate building process.

11. 'Apply Transforms'. This button is used to calibrate the pelvis to its surrounding environment. If the user is only using the template pelvis, this button should be used whenever the pelvic model has been rotated or translated in the software using the tools seen in Figure 11. If the user imports their own pelvic model, this button should be used before the plate placement process begins. 

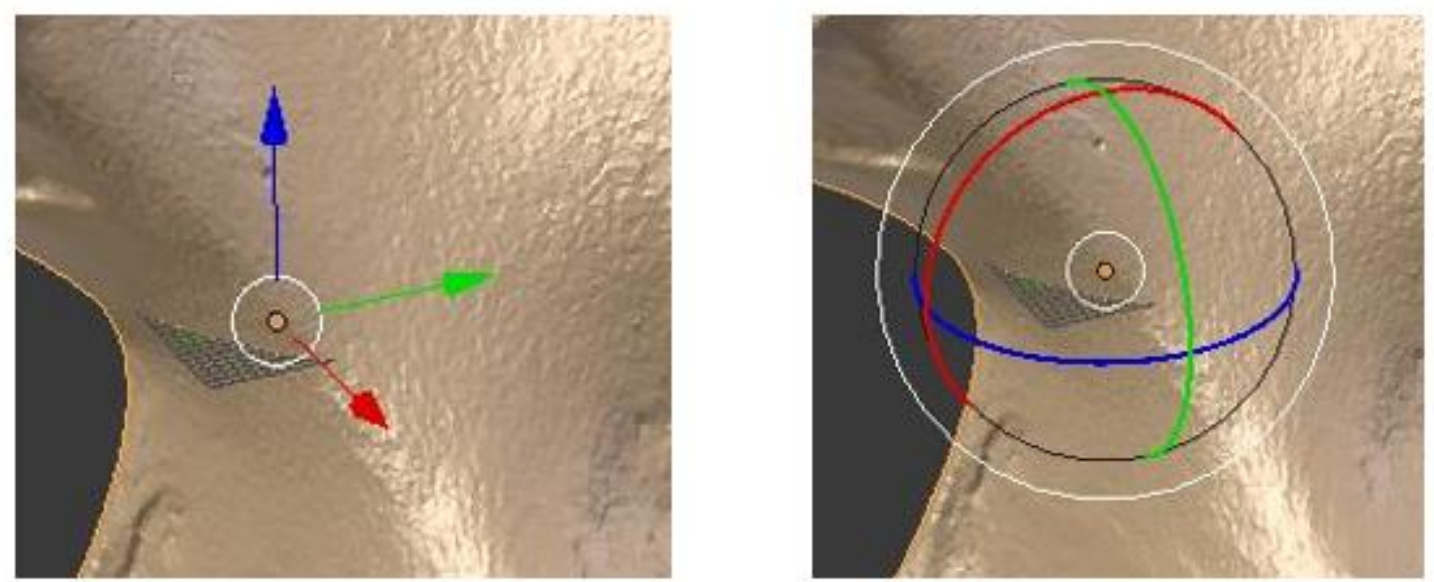

Figure 11 - Rotating and Translating the Pelvis. Use of either of these tools will cause the pelvis to become unaligned from its surrounding environment. It will not physically look out of place but plates that are attempted will not be properly oriented.

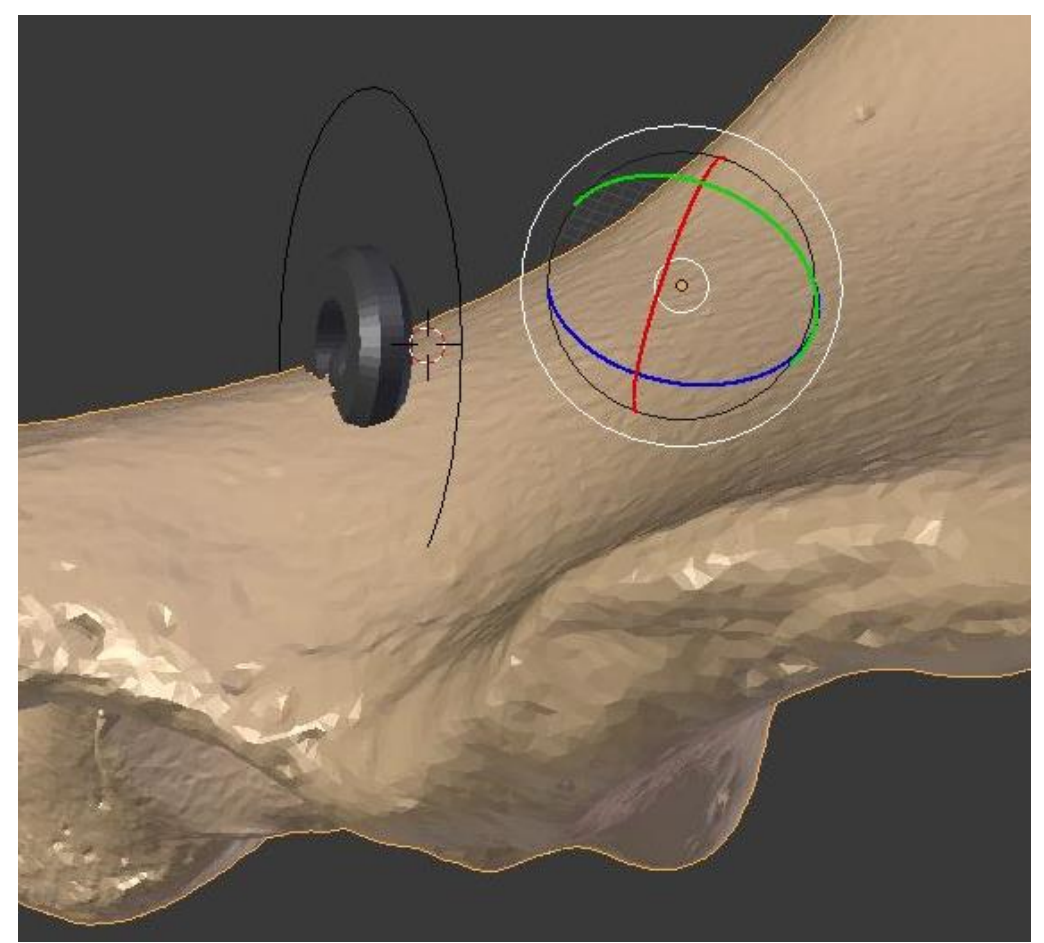

Figure 12 - Unaligned Pelvis Creates Skewed Screw Hole. The pelvis in this photo was rotated but the 'Apply Transforms' function was never used so the screw hole placed on this surface is skewed. 
D. General Process for Template Creation

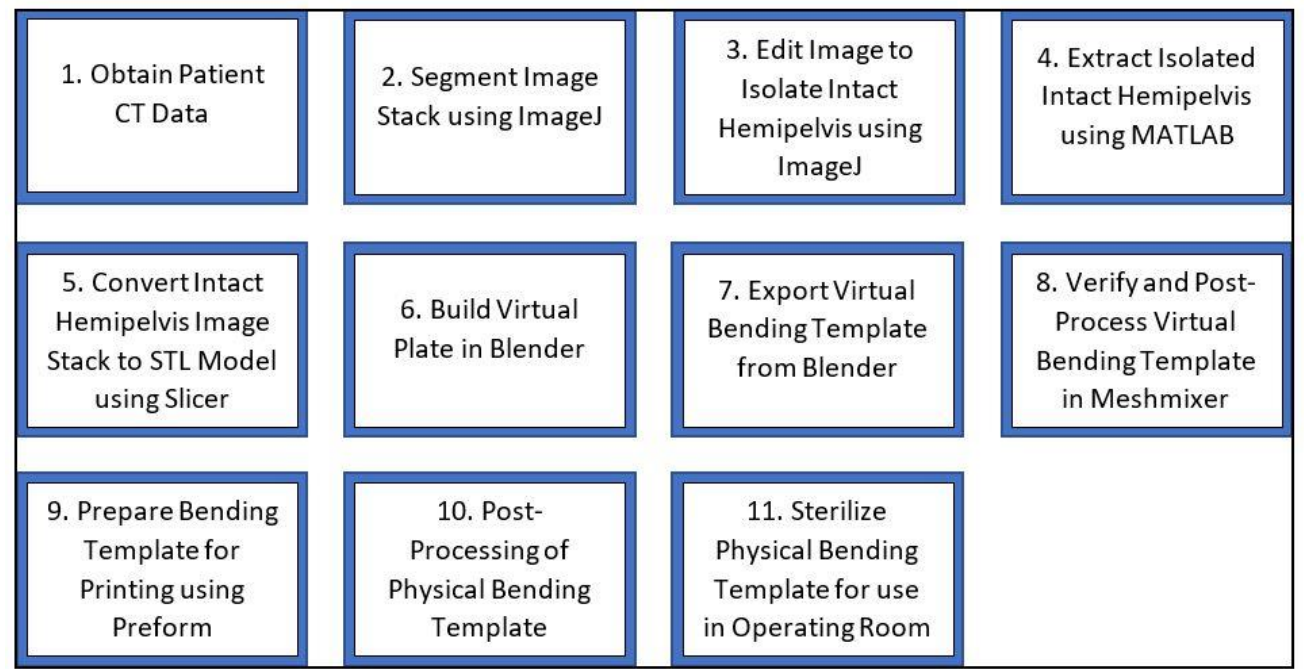

Figure 13 - Flowchart for Designing a Bending Template.

1. Obtain Patient CT Data. Images from CT scans

are generated in the DICOM format which contains both the

images and headers with associated information. Images

obtained from the University of Louisville Synapse database

often have no file extensions since they are opened in an

included viewing program. Using the CT stacks beyond the

included viewing program requires the user to manually add

the file extensions or use the follow batch code to convert

them:

$$
\begin{aligned}
& \text { @Echo Off } \\
& \text { ren } * * . d c m
\end{aligned}
$$

These stacks of DICOM images are opened in ImageJ

through the 'Import' $->$ 'Image Sequence...' menu. Before performing any further steps, the user should verify the voxel dimensions in the image stack through 'Image' -> 
'Properties' and making note of 'Pixel Width', 'Pixel Height', and 'Voxel Depth'. These values will be needed later to ensure an accurate 3D reconstruction in performed.

2. Segmenting the Image Stack. Segmentation is the process of extracting the tissue of interest from the surrounding tissues. For this thesis, bone was the tissue of interest and threshold segmentation was performed. In CT scans, pixels in the image are assigned a grayscale value from 0 to 255. The pixels representing the least dense tissue, usually air, are assigned a value of 0 , or pure black. The pixels representing the densest objects, usually bone or titanium, are assigned a value of 255, or pure white. The pixels representing other tissues in the image will be assigned a value between 1-244 that is a shade of gray. In threshold segmentation, a threshold value is set to determine what tissue to keep and what tissue to ignore. This creates a binary image by setting any pixel below the threshold value to 0 and any pixel above the value to 255 . The process itself is somewhat subjective and other factors, such as the partial volume effect, play a role in making it difficult. The partial volume effect occurs in CT scans when very dense tissue is located next to much softer tissues. Because the resolution of the image obtained in a 
CT scan is limited, it is possible for density transitions to occur within the boundaries of the pixel. When this occurs, the computer will average the densities of the two tissues and assign that color to the pixel. During segmentation, this can create difficulties because the computer may not have the resolution to recognize that a boundary exists between two fragments of a bone or a joint. For all image stacks processed in this thesis, a threshold was chosen to balance the intact hemipelvis being completely enclosed with the reduction of noise. This method is easily verified within ImageJ by using the 'fill hole' function and verifying that the intact hemipelvis has been filled in on all slices of the stack.

\section{Edit Image Stack to Isolate Hemipelvis. After} segmentation, the goal is to obtain the intact hemipelvis so that it can eventually be mirrored and used as the reconstructed pelvis on which the plate can be bent. The partial volume effect complicates this process as it makes individual bones often appear as if they are connected. This is common at the sacroiliac (SI) joint between the iliac wing and the sacrum of the pelvis. To separate the iliac wing, the paintbrush effect is used to manually erase white pixels from the joint space. This method does not 
compromise the accuracy of the pelvis since material can be preferentially removed from the sacrum to disconnect the two bones. Additionally, the iliac wing portion of the SI joint is not a location for reconstruction plates making any material removed from it inconsequential. Another method for separating bones involves eroding the image. During erosion, a single pixel is deleted from the perimeter of all objects, making it an excellent way to remove noise from the image while not compromising important structures. After the bone of interest is extracted, it will be dilated to bring the bone back to its original size.

4. Extract Isolated Hemipelvis. After segmentation and initial edits within ImageJ, the stack of $\mathrm{CT}$ images is saved as a TIFF file. This TIFF file is then opened in MATLAB and edited using the previously described function. After the intact hemipelvis has been separated, any erosions performed in ImageJ or MATLAB are corrected with a similar dilation.

5. Convert Hemipelvis to STL Model. The isolated hemipelvis can now be opened in slicer to convert it from a stack of binary images into a STL file. The TIFF file is 
imported through the 'Load Data' button while in the 'Welcome to Blender' module. To confirm the correct dimensions of the voxels are being used, the user should change the dimensions in the 'Volumes' module under the 'Volume Information Tab'. Slicer assumes that all imported volumes are isotropic with $1 \mathrm{~mm}$ dimensions. This should be changed by the user since most clinical scans will have length and widths of $0.488 \mathrm{~mm}$ and can range from $0.5 \mathrm{~mm}-3 \mathrm{~mm}$ in height. This information can be obtained from a DICOM image stack in Image J as described before. With the proper dimensions applied, the image stack is rebuilt as a 3D model. The user can now enter the 'Editor' module and should confirm the default label map. The user will have to threshold the image again by pressing the 'ThresholdEffect' button. Since the image imported is 8-bit and already binary, the lower threshold range should be 1.0 and the upper should be 255.0. The user now should click 'Apply' and then 'MakeModeleffect'. The user should specify a new model name, ensure the 'Smooth' box is checked, and click 'Apply'. There will now be a model of the pelvis in the upper right screen. The user can export this model through the 'Save' button, de-selecting all items, and reselecting only the file with the model name the user specified and a ".vtk" extension. The user should use the drop-down menu to 
change ".vtk" to ".stl", specify the output directory, and press 'Save'. With the STL model created, the pelvis can now be opened within Blender and the extension can be used to build a virtual reconstruction plate.

6. Build Virtual Plate in Blender. The STL file of the intact pelvis should be imported into Blender through 'File' $->$ 'Import' $->$ '.stl'. Once imported, the user will need to manually rename their pelvic model to "Pelvis" under the scene tab on the right-hand side of the screen. Much of the software's underlying process involves referring to an object named "Pelvis" and a lack of that object will prevent the software from working properly. After importing the desired pelvis model, it is wise to use the 'Apply Transform' button included in the side panel to ensure that the new pelvis is properly aligned. The user can adjust their view of the pelvis by holding the middle mouse button. Occasionally, the user will not be to obtain the desired view through the mouse alone and the model itself will need to be rotated. \{image showing how to do this.\} If the model is rotated using this method, the 'Apply Transforms' button must be clicked to reorient the software. 
If desired, facture lines can be transferred to the intact pelvis using the Grease Pencil utility. To place a fracture line after the pelvis is imported, orient the pelvis so that the desired surface is easily accessible. Open the Grease Pencil side panel, check the box labeled 'Continuous Drawing', and select 'Surface' under the stroke Placement header. To change the Grease Pencil color, Press ' $N$ ' on the keyboard to open the settings menu, press the 'New Layer' button under the Grease Pencil Layers header, click the black box under the Tint header and slide the bar next to $\mathrm{R}$ to make the line red. When ready to place the fracture line, the user should click the 'Draw' button under the Draw header in Grease Pencil side panel. Lines can now be drawn onto the surface of the pelvis to represent the location of fracture lines. When done, the user can click anywhere outside of the window to stop drawing.

When ready to place the virtual plate, the user should open the Pelvis side panel. The user can then place the 3D cursor at the desired location and press the 'Place Screw Hole' button to begin construction of the virtual plate. If at any point the user wishes to remove the last screw hole placed, they can press CTRL-Z to undo their last action. If the user wishes to restart, they may press the 
'Erase Everything' button located in the Pelvis side panel to remove everything except the pelvis and any drawn fracture lines.

7. Export Virtual Bending Template. When the user is ready to export the newly created template, they should press the button labeled "Export Screws as .stl". If the user only needed to create a single template, they should now save the file through 'File' -> 'Save as...' and may close Blender. If the user wishes to create a second template on the same pelvis, they should press the button labeled "Export Plate as .stl". The plate visible on the pelvis is now a static object that cannot be edited further. The user should change the value located in the 'Filename' box on the side panel and can then begin to build subsequent plates.

\section{Post-Processing of Virtual Template. After} saving the temple from Blender, the user should open the template in Meshmixer for final processing. The object should be oriented with the spheres and pelvis surface facing upward by using the 'Edit' -> 'Transform' tool. The user should also take advantage of the 'Analyze' ->

'Inspector' tool to ensure there are no structural flaws 
with their part. If the tool reports the spheres as problems \{image\} the user should not click "Auto Repair All' and should instead manually click through the repair features, ensuring that the spheres remain intact. The user can perform additional editing to the object by extruding surfaces to make the template easier to handle while in the operating room\{image\}. When the user is satisfied with the condition of their STL template, it can be exported from Meshmixer using 'File' -> 'Export'.

9. Preparing Template for Printing. To print the bending template, the user should open Preform. The user will need to confirm the printer and correct material are being used. For this thesis, the printer being used is always the Form 2. If the part is being printed for nonclinical uses, then any material may be used. If the part is being tested for clinical use, only Dental SG should be used. The user can press 'Apply' to confirm settings and bring up their build tray. To import their template, the user will have to drag their template file to the build tray. The user can then modify the orientation of the template if necessary to ensure the spheres and pelvis surface are facing upwards. The user should confirm support structure through 'Supports' -> 'Generate All' located on 
the left-hand panel. Finally, the user should connect their computer to the Form 2 printer, save their print file in ".form" format, and press the orange 'Start a Print' button. This will bring up a window for the user to select their printer and confirm their action. The USB cable connecting the user's computer and the Form 2 printer can now be disconnected. The user can now use the control interface on the printer to start the print.

\section{Post-Processing of Physical Template. After}

the print is finished, the user may remove the print platform from the printer and remove the part from the print platform. The part should then be washed in IPA for 10-20 minutes and transferred to a UV curing station for 10-30 minutes. After curing, the part is removed from the curing station and support structures are removed [39].

\section{Sterilization and Bending. If the part was} printed in Dental SG and is intended to be used clinically, it should be sterilized before entering the operating room. Sterilization of the template can be done using an autoclave and one of three possible time/temperature combinations. Acceptable combinations are as follows: $134^{\circ} \mathrm{C}$ for 6 minutes, $121^{\circ} \mathrm{C}$ for 15 minutes, or $138^{\circ} \mathrm{C}$ for 3 
minutes. The part should be kept free of particulate contamination between printing and sterilization and should only be sterilized using the autoclave available in the surgery department that the template will be used in. After the template is used for bending, it should be washed and re-sterilized before dimensional verification.

\section{B. Proof of Process and Size Verification}

The following work was done validate that the process of creating a bending template from CT data and using it to accurately bend a plate.

The CT scan data used to create the template pelvis for this proof of process was obtained from the Laboratory of Human Anatomy and Embryology, University of Brussels (ULB), Belgium. The scan took place in 3 sequences:

Crest/Ilium, Acetabulum, and Ischium/Pubis. For all sequences, the images were $512 \times 512$ pixels with each pixel representing a square of $0.488 \mathrm{~mm}$. For the crest/ilium and ischium/pubis sequences, slice height was $1 \mathrm{~mm}$. For the acetabulum, slice height was $0.5 \mathrm{~mm}$. ImageJ was used to rebuild the three sequences as a single file. For the crest/ilium and ischium/pubis sequences, the images were 
resized to double their normal length with no interpolation. The product of this was that, for these two sequences, each slice was duplicated but all files could now be combined and reconstructed with a slice height of $0.5 \mathrm{~mm}$. The files were then concatenated in ImageJ, converted to 8-bit, and segmented at a threshold of 37. To remove noise, the entire stack was eroded once in ImageJ.

The TIFF stack was then imported into MATLAB and the left iliac wing was isolated from the remaining noise. No morphological operations were performed in MATLAB. The text output of the process used to extract the pelvis from surrounding noise is located in Appendix I. After the pelvis was isolated, it was brought back into ImageJ and dilated to bring it back to its original size. This TIFF stack was then imported into slicer to create a STL model of the pelvis. The dimensions were adjusted to have a length and width of $0.488 \mathrm{~mm}$ and a height of $0.5 \mathrm{~mm}$. After adjusting the dimensions, the threshold limit was set to 1.0 and 'MakeModelEffect' was used to convert the model into a solid body. After the conversion, the STL file was exported through 'Save' and changing the default '.vtk' format to '.stl'. 
The STL file of the pelvis was then opened in Blender, oriented, and the 'Apply Transforms' button was used to properly align the model. Figure 14 shows the input from an orthopedic trauma surgeon detailing where a posterior wall and an ilioinguinal plate should be placed and the virtual plates created within the software.
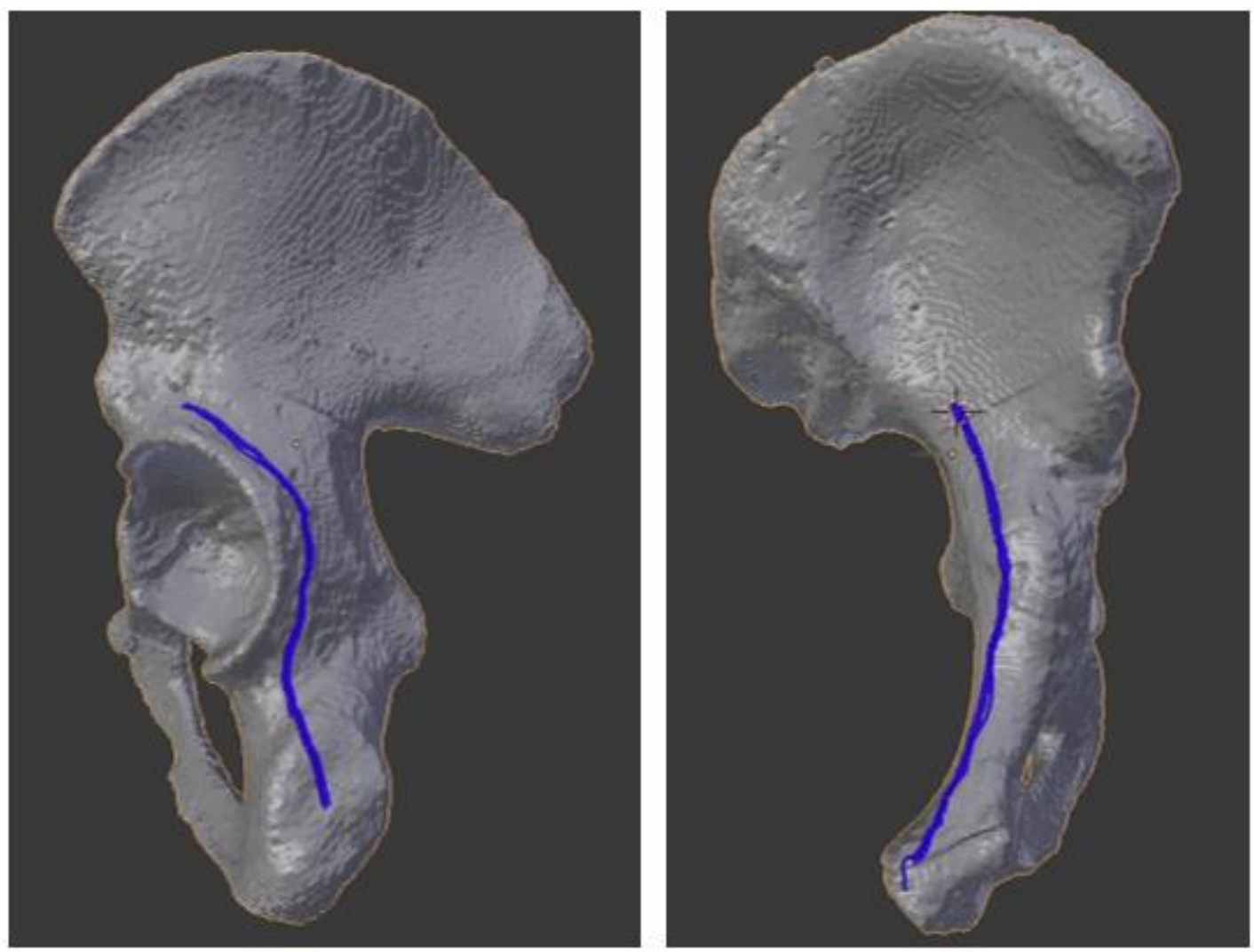

Figure 14 - Surgeon Specified Locations of Plates. The 'GreasePencil' tool was used to annotate the locations of the Posterior Wall (left) and Anterior Brim (right) plates.

These plate locations were chosen because posterior wall plates often have the most complex geometries and 
ilioinguinal plates are capable of displacing reduced fragments if they are not properly bend before being secured. The surgeon specified that posterior wall plates are often 6-8 holes and ilioinguinal plates are 8-10 holes depending on the patient's gender and size.

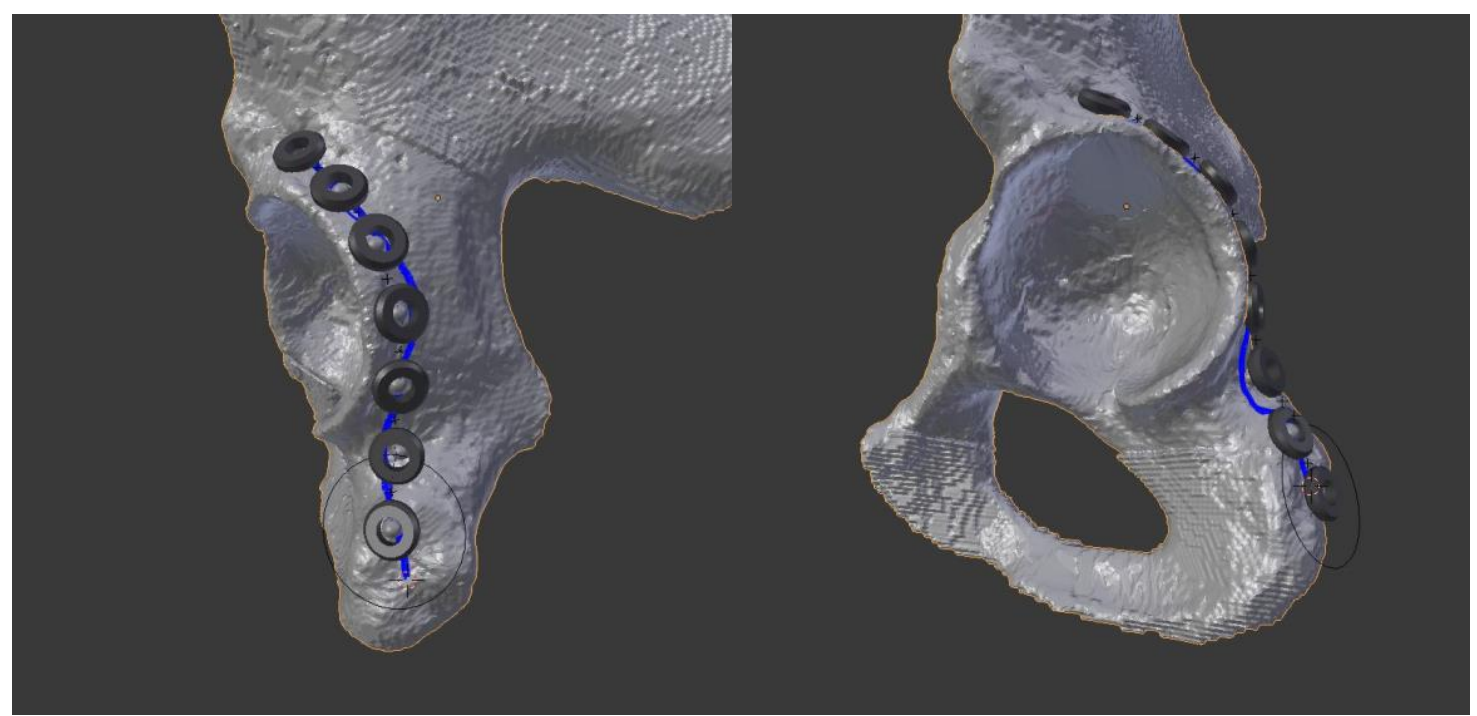

Figure 15 - Surgeon Specified Posterior Wall Plate.

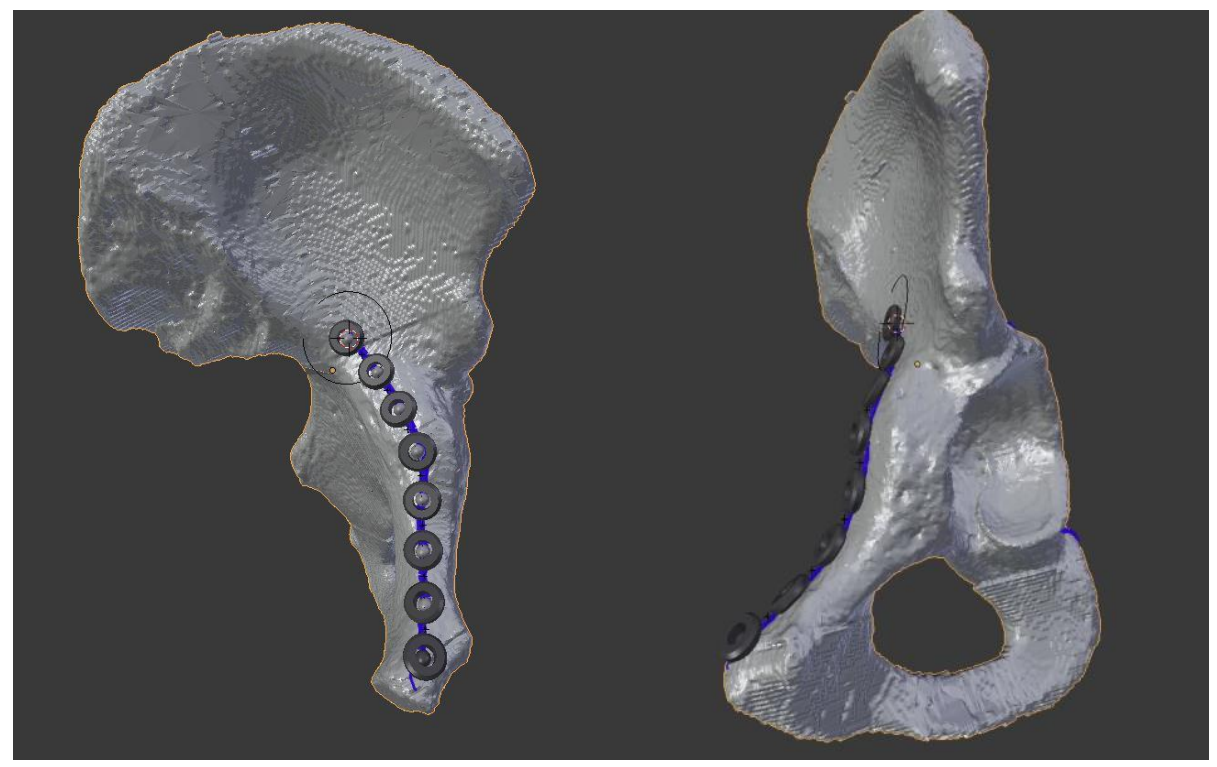

Figure 16 - Surgeon Specified Anterior Brim Plate. 
The bending templates from these plates were exported from Blender and opened in Meshmixer for final processing. In Meshmixer, holes in the mesh were filled, portions of the mesh were smoothed, the part was reoriented, and a stand was created for the parts so that they could be set upright. The vertical portion of the stand was created by placing circular stamps and extruding them down. The bases of the templates were created using Solidworks. Figure 17 shows the bounding box of the two plates and Table I shows the dimensions of the plates and the dimensions of their corresponding base plates. By creating a base with similar dimensions to that of the template, the base itself can be measured for dimensional accuracy 


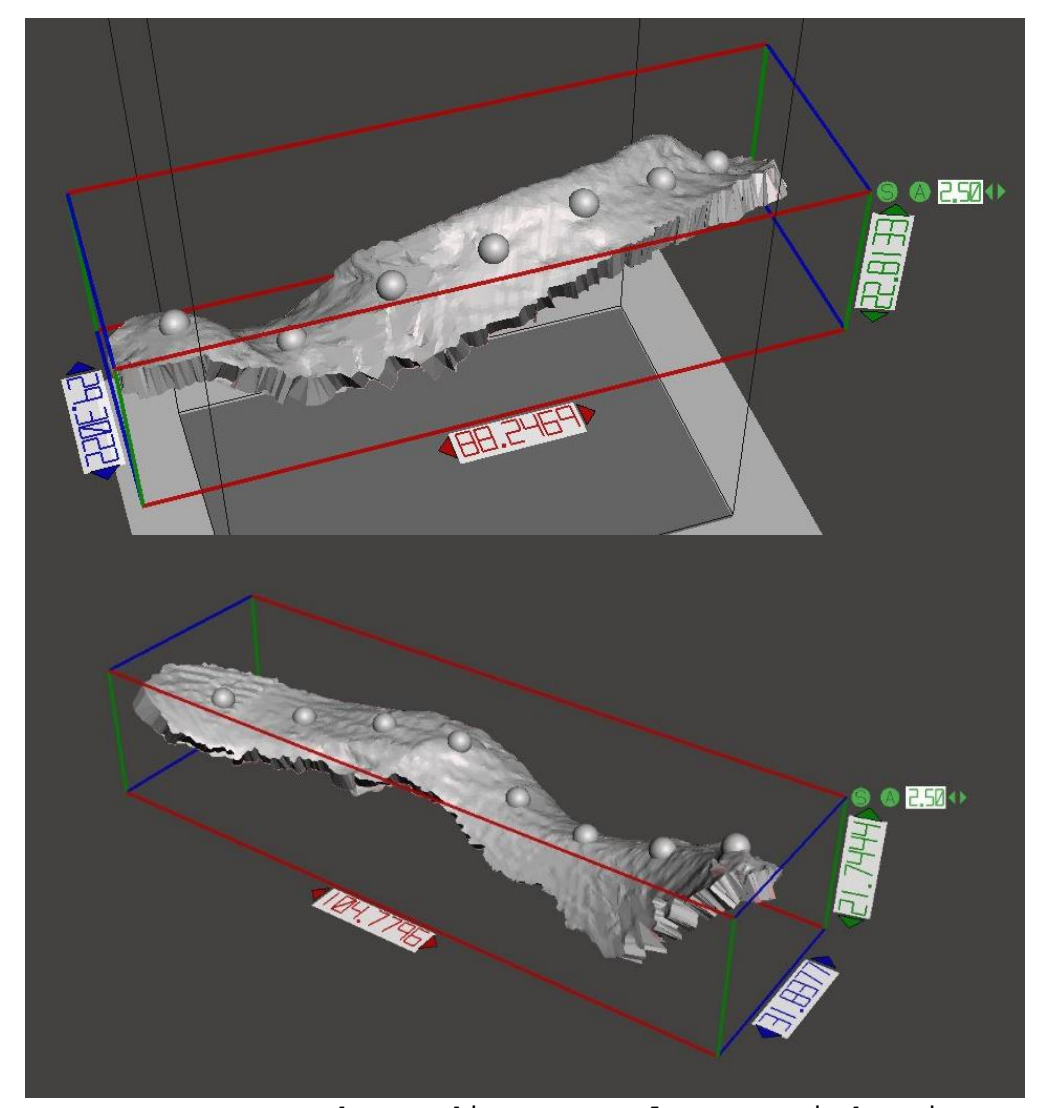

Figure 17 - Pre-Processed Bending Templates with Dimensional Bounding Box. The Posterior Wall template is positioned on top and the Anterior Brim template is positioned on bottom.

Table I - Dimensions of the Plates and Bases

\begin{tabular}{|c|c|c|c|c|}
\hline & $\begin{array}{c}\text { Meshmixer } \\
\text { Length } \\
(\mathrm{mm})\end{array}$ & $\begin{array}{c}\text { Meshmixer } \\
\text { Width (mm) }\end{array}$ & $\begin{array}{c}\text { Baseplate } \\
\text { Length } \\
(\mathrm{mm})\end{array}$ & $\begin{array}{c}\text { Baseplate } \\
\text { Width (mm) }\end{array}$ \\
\hline $\begin{array}{c}\text { Posterior } \\
\text { Column }\end{array}$ & 88.25 & 29.30 & 88.00 & 29.00 \\
\hline $\begin{array}{c}\text { Anterior } \\
\text { Brim }\end{array}$ & 104.78 & 31.84 & 104.00 & 31.00 \\
\hline
\end{tabular}




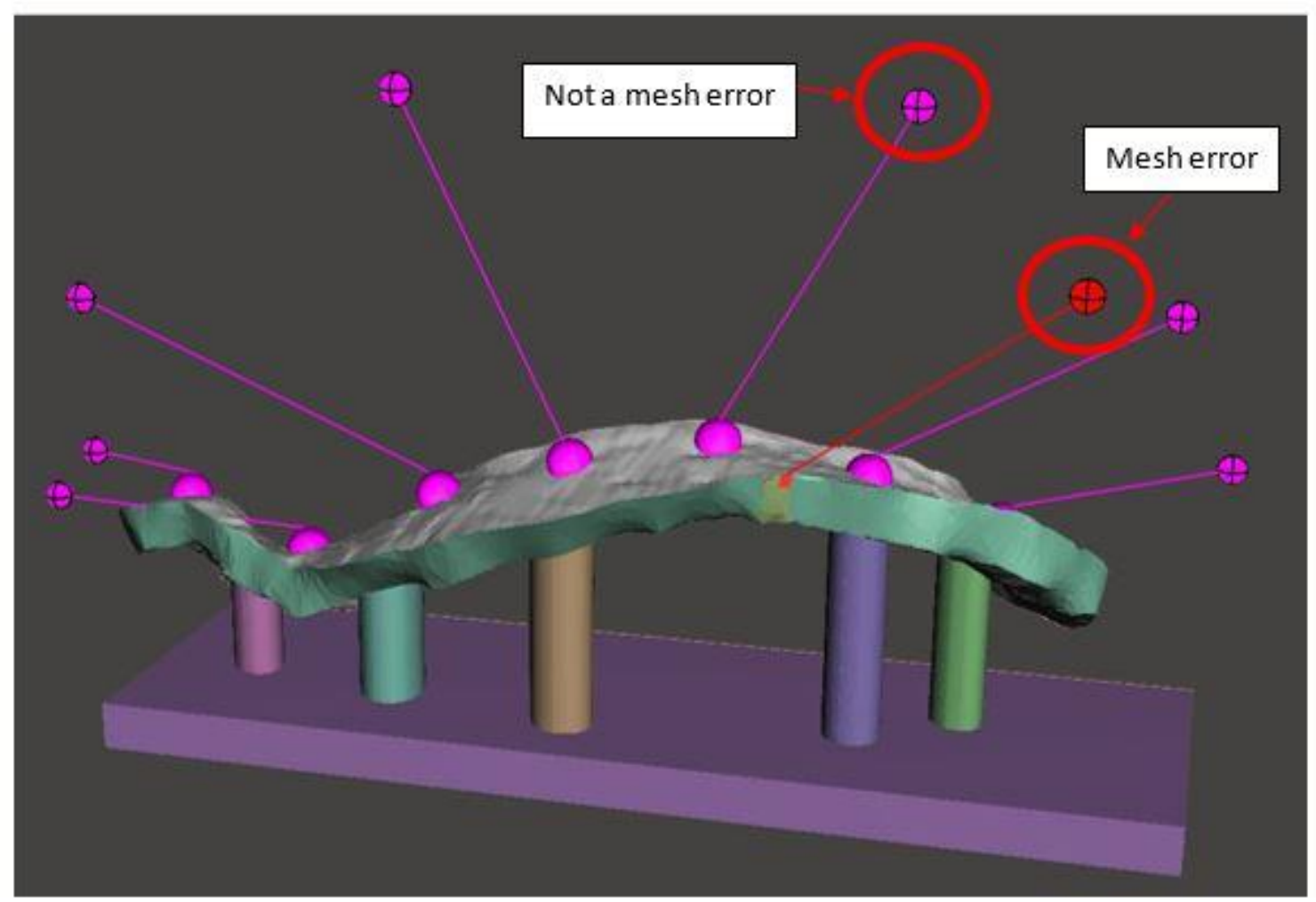

Figure 18 - Post-Processed Posterior Wall Virtual Template. This image shows the bending template with its vertical support and baseplate before the single error was repair and it was exported from Meshmixer. Meshmixer often attempts to classify the screw landmarks as mesh errors.

\section{After the bending templates had been modified in}

Meshmixer, they were imported into Preform. The print slice resolution was set to $50 \mu \mathrm{m}$ and the material used was Clear. An isotropic $15 \mathrm{~mm}$ cube was also included in the build tray for further dimensional verification of the parts. To conserve material, only the posterior wall plate was printed. Since the posterior wall has the most complex bends, this represents the worst-case scenario of a bending template that would need to be made. Location of the 
template and the cube within the build tray can be seen in Figure 19.

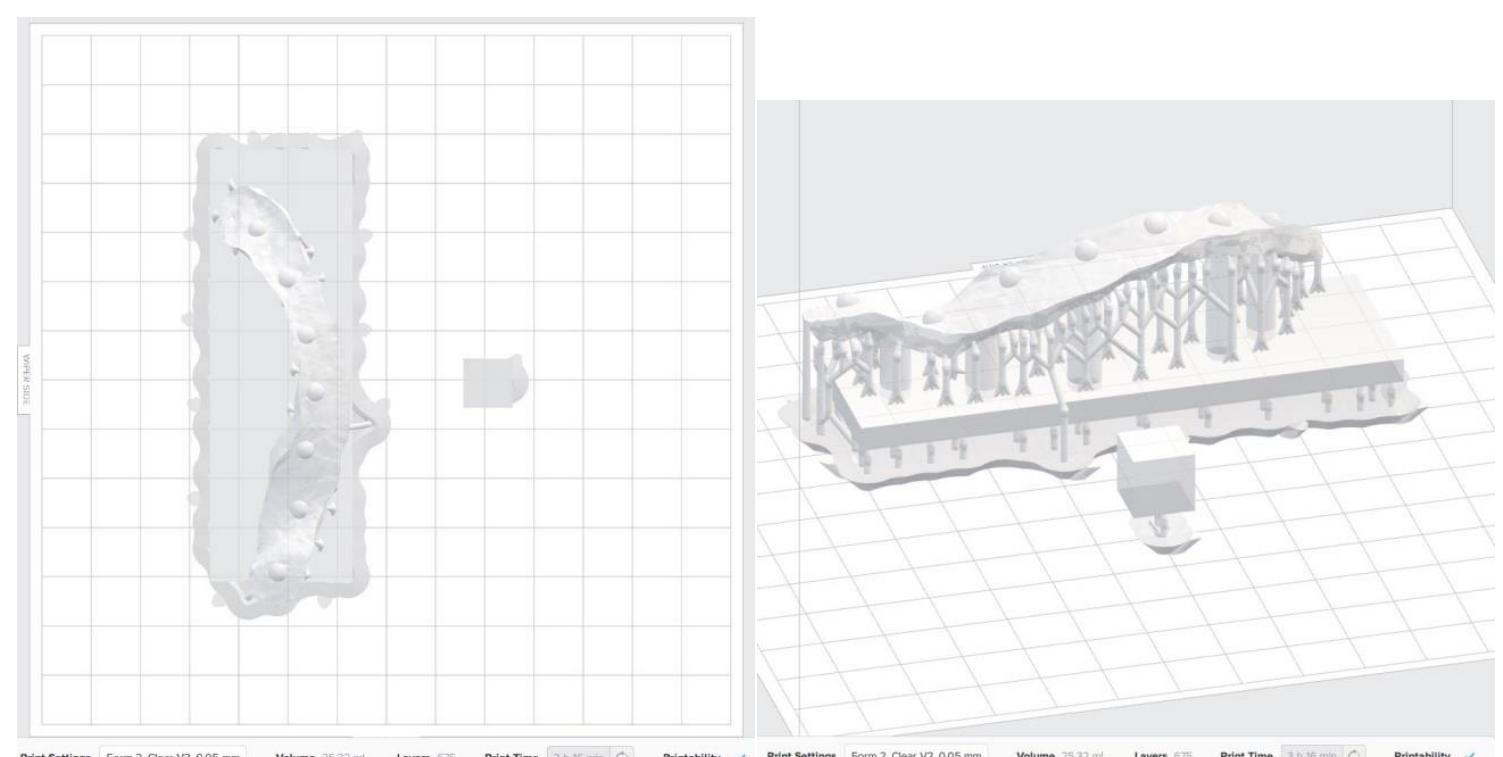

Figure 19 - Two Views of the Build Tray. The left image shows the location of the two parts on the build tray. The right image shows the support material needed for the part to print successfully.

After the build finished, all parts were washed in isopropyl alcohol baths for 10 minutes. Parts were then placed in a UV curing station for a total of 30 minutes. After curing, the support structures were removed and the dimensions of the base of each template and the 3 faces of the calibration cube were measured. Finally, reconstruction plates were bent by an orthopedic surgeon using the bending template as a guide. 


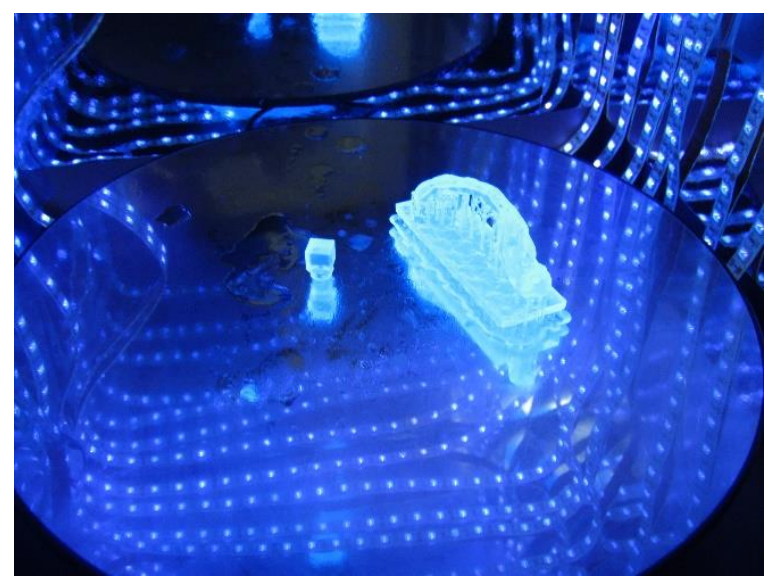

Figure 20 - Template in UV Curing Station.

\section{Segmentation Threshold and Size of Pelvis}

For this work, segmentation of the bone from

surrounding bone was done using threshold segmentation. For the proof of process bending template design, a threshold was chosen so that the cortical bone was a single, enclosed entity. Confirmation of the correct threshold was confirmed using the 'fill' feature in ImageJ and ensuring the entire area within the cortex of the bone was filled in all images within the stack. For the proof of process, a threshold of $37 / 255$ was used. This method produced adequate results but is inherently very subjective. Additionally, other users may wish to use higher threshold values in order to remove noise or lower threshold values in order to capture thin features of bone. These variations in threshold value will result in slight changes to the morphology and size of the reconstructed pelvis due to the partial volume effect. To 
account for users who may choose to use a lower or higher threshold value than that described in this work the pelvis was segmented at different threshold values, reconstructed as an STL file, and differences between the models were examined. Additionally, the model used for the proof of process was actually composed of $3 \mathrm{CT}$ scans. Two of the scans, the most superior portion of the pelvis, the iliac crest and the most inferior portion, the ischium, were done using $1.0 \mathrm{~mm}$ slice thickness and the acetabulum was done using $0.5 \mathrm{~mm}$ slice thickness. This provided a very accurate model for our work but is not representative of the $3 \mathrm{~mm}$ scans that are often used for diagnosing pelvic fracture. For this reason, we will compare the reconstruction of the $1 \mathrm{~mm}$ and $0.5 \mathrm{~mm}$ slices at the original threshold of 37 with a $3 \mathrm{~mm}$ slice of the same pelvis. In order to create $3 \mathrm{~mm}$ slices, only every third slice will be used in the $1.0 \mathrm{~mm}$ scans and only every sixth slice will be used in the $0.5 \mathrm{~mm}$ scan .

A total of three segmentations were performed at 31, 37, and 43. 37 was chosen since it was used for the proof of process model and 31 and 43 were chosen since they were six units below and above respectively. All three models were reconstructed using the $1 \mathrm{~mm}$ and $0.5 \mathrm{~mm}$ slices and the threshold 37 model was additionally reconstructed as a $3 \mathrm{~mm}$ 
slice thickness model. The fill feature was used on all image stacks and stacks containing voids were manually repaired to create a continuous, enclosed feature. Reconstruction of the image stacks to STL models was performed within Slicer, pixel dimensions were $0.488 \mathrm{mmx} 0.488 \mathrm{~mm}$ and voxel height was $0.5 \mathrm{~mm}$ for all slices except the $3 \mathrm{~mm}$ reconstruction. After reconstruction, the differences between the models were compared using CloudCompare. The STL models are imported into CloudCompare and are resampled to allow for the greatest accuracy in distance detection. Resampling is done through 'Edit' -> 'Mesh' -> 'Sample Points' and 1,000,000 points were used. After both objects had been resampled, they were aligned to each other through 'Tools' -> 'Registration' -> 'Fine Registration (ICP)'. This registration method required that one model be set as the 'Aligned' and the other as the 'Reference'. For this work, both models were tested in both roles to determine if aligned would play a role and the RMS value, or the accuracy of the alignment, is reported. After alignment, both objects were selected and the distance between the points of each cloud were calculated using 'Tools' -> 'Distances' -> 'Cloud/Cloud Dist.'. The results are more accurate when the 'Compared' object is set as the larger of the two objects and the 'Reference' object is the 
smaller. However, since the pelvis is a complex model, both models were used in both roles and the results of each were recorded. For the comparison of pelvis models, each comparison was done four times to allow both models to serve as the 'Aligned' and 'Compared' Models. The software reports the average distance that the two objects deviate by but also organizes the data into distance bins that allow the user to see relatively how many points deviated by certain distances. The software also reports minimum and maximum distances.

To illustrate how this software works two cubes, A and B, were created with $10 \mathrm{~mm}$ and $12 \mathrm{~mm}$ side lengths respectively. Figure 21 shows the two cubes as points clouds aligned with both their centers at point $(0,0,0)$.

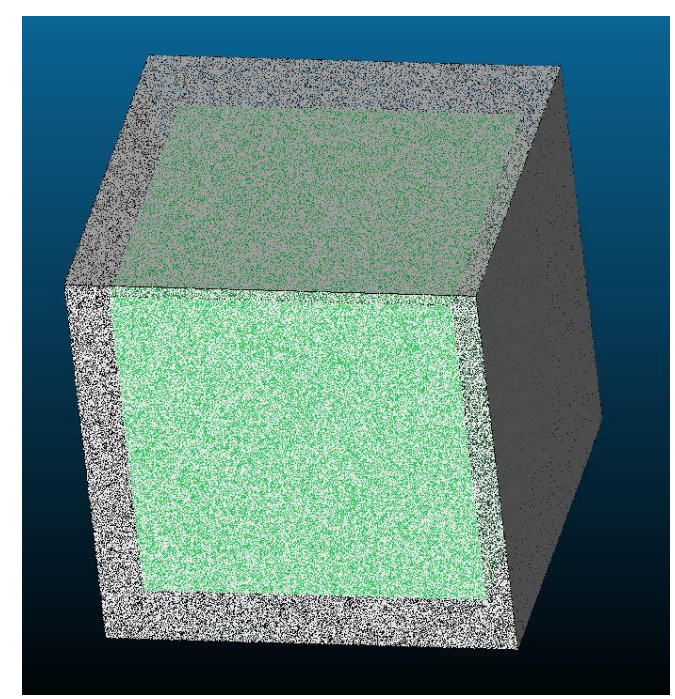

Figure 21 - Two Cubes Aligned. Showing the green box of Cube A, $10 \mathrm{~mm}$, inside the gray point cloud of Cube $\mathrm{B}, 12 \mathrm{~mm}$. 
Before the distance calculations are run through the computer, several distances can be calculated by hand. In Figure 22 we see a two-dimensional representation of how the cubes are positioned in one another.

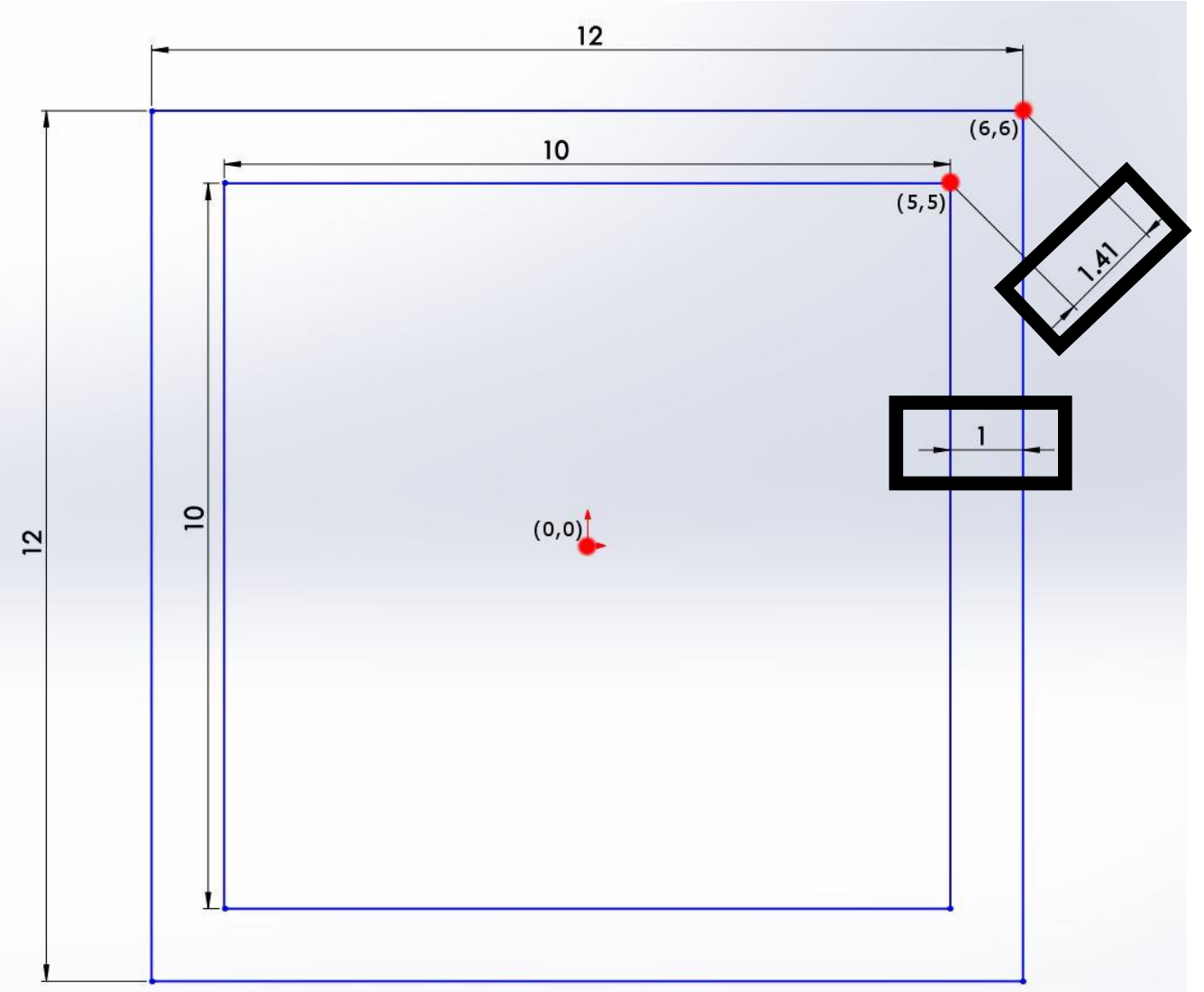

Figure 22 - Cubes A and B as Squares. Cube A, 10mm, and Cube B, $12 \mathrm{~mm}$ both have their center at $(0,0)$. The two black boxes show the dimensions, in $\mathrm{mm}$, between the walls and the corner points of the two squares.

Equation 3 shows that since cube $A$ and cube $B$ have the same center, the distance between their parallel walls can be calculated as $1.0 \mathrm{~mm}$. Equation 4 shows how Pythagorean's theorem is used to calculate the distance between the edges of the two cubes as approximately $1.41 \mathrm{~mm}$. Finally, 
Equation 5 uses Pythagorean's theorem to calculate the distance between the corner vertices of the two cubes as approximately $1.73 \mathrm{~mm}$.

$$
\begin{gathered}
\frac{(10.0-12.0)}{2}=1.000 \mathrm{~mm} \\
\sqrt{(5.0-6.0)^{2}+(5.0-6.0)^{2}}=\sqrt{2}=\sim 1.414 \mathrm{~mm} \\
\sqrt{(5.0-6.0)^{2}+(5.0-6.0)^{2}+(5.0-6.0)^{2}}=\sqrt{3}=\sim 1.732 \mathrm{~mm}
\end{gathered}
$$

With these known distances, we can now better understand the output that the software provides. Figure 23 shows a histogram of the distances and Figure 24 shows the complete colormap as well as the previously calculated distances emphasized. Table II shows the parameters for this example. It was assumed that since both objects were isotropic cubes, the object assigned as 'Align' would not make a large difference and this is confirmed with the resulting RMS values of 1.00 . The data represented in Figures 23 and 24 are highlighted in yellow in Table II. 


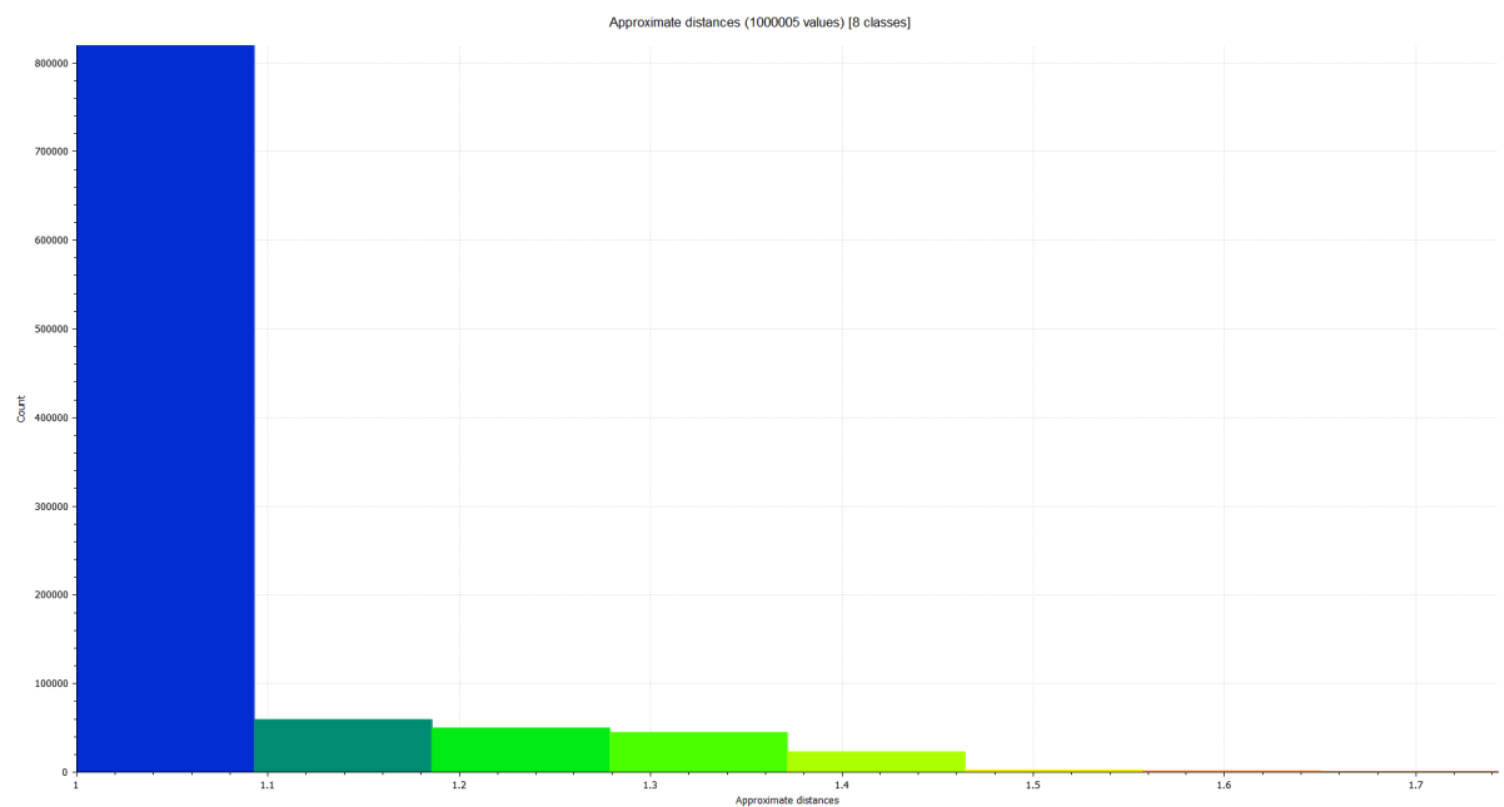

Figure 23 - Histogram of the Distances Between Points.

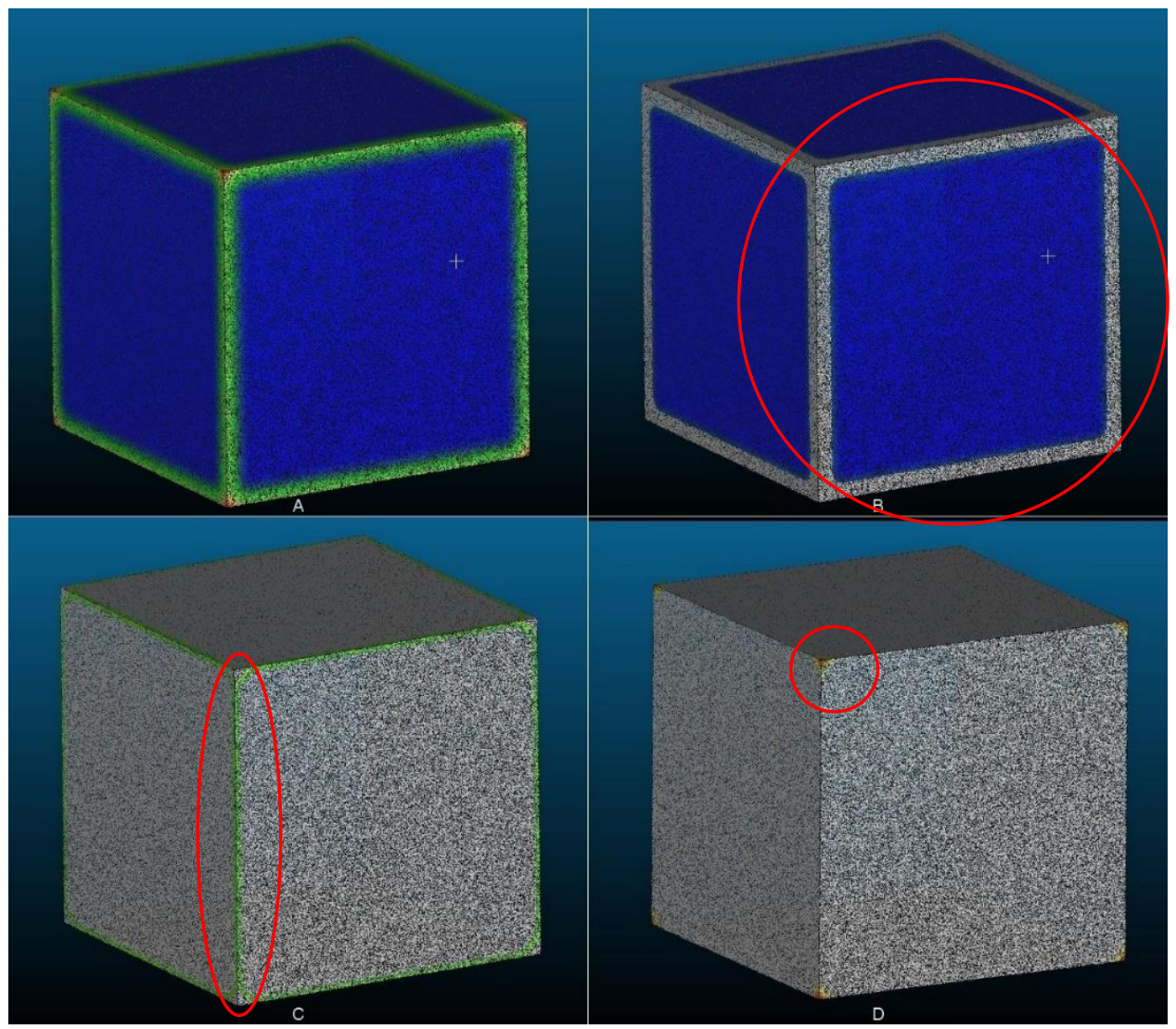

Figure 24 - Montage of Distance Colormap. ' $A$ ' shows the entire colormap on the larger cube, ' $B$ ' shows distances of 1.0-1.1 mm, ' $C$ ' shows distances of $1.30-1.45 \mathrm{~mm}$, and ' $\mathrm{D}$ ' shows distances $1.45-1.75 \mathrm{~mm}$. 
Table II - Parameters of the Cube Example.

\begin{tabular}{|c|c|c|c|c|c|c|c|}
\hline aligned & reference & compared & reference & RMS & avg $(\mathbf{m m})$ & std dev $(\mathbf{m m})$ & $\mathbf{m a x}(\mathbf{m m})$ \\
\hline $10 \mathrm{~mm}$ & $12 \mathrm{~mm}$ & $10 \mathrm{~mm}$ & $12 \mathrm{~mm}$ & 1.0026 & 0.9999 & 0.0235 & 1.0569 \\
$10 \mathrm{~mm}$ & $12 \mathrm{~mm}$ & $12 \mathrm{~mm}$ & $10 \mathrm{~mm}$ & 1.0027 & 1.0495 & 0.109 & 1.7503 \\
\hline
\end{tabular}

CloudCompare reported the average distance between cubes as $0.9999 \pm 0.0235 \mathrm{~mm}$ and the maximum distance as 1.750 . The circled portions in Figure 24 show that the output from the software agrees well with our previously calculated values. It is possible that the reported maximum distance did not perfectly agree with our calculated maximum distance of $1.732 \mathrm{~mm}$ because of the process of resampling the STL file into a point cloud. The data in Table II confirms that the 'Compared' model plays a large role in obtaining accurate measurements. The maximum distance reported when the $10 \mathrm{~mm}$ cube was the 'Compared' model was 1.06mm, far lower than our calculations showed. Knowledge of how CloudCompare calculates and displays distances will allow for easier interpretation when the pelvic STL models are compared. 


\section{RESULTS}

\section{A. Initial Plate Designs}

\section{Iterations of bending templates for the posterior}

wall plate and pelvic brim can be seen in Figure 25. A reconstruction plate bent to match one of the later iterations in seen in Figure 26.

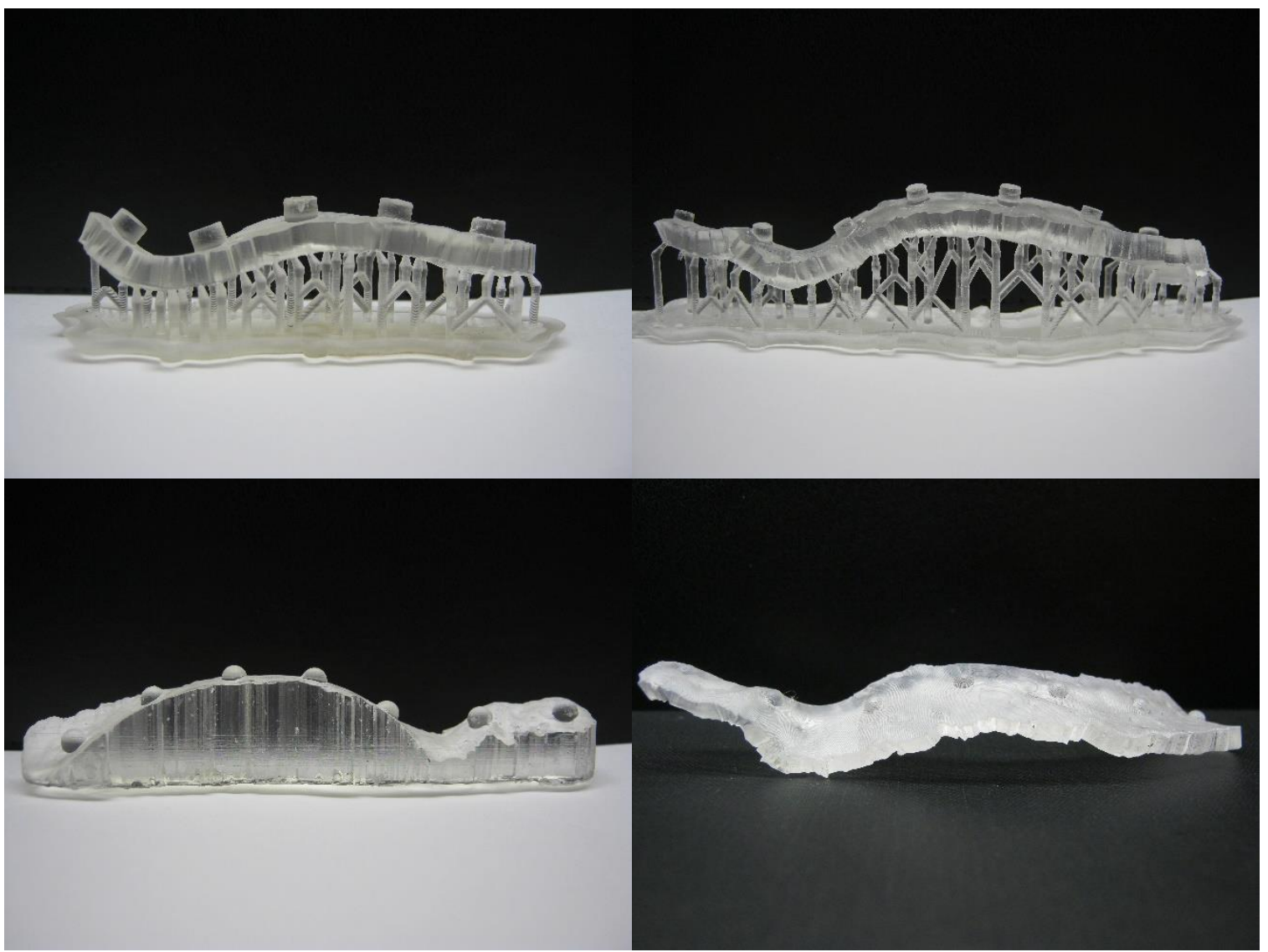

Figure 25 - Several Initial Bending Templates. 


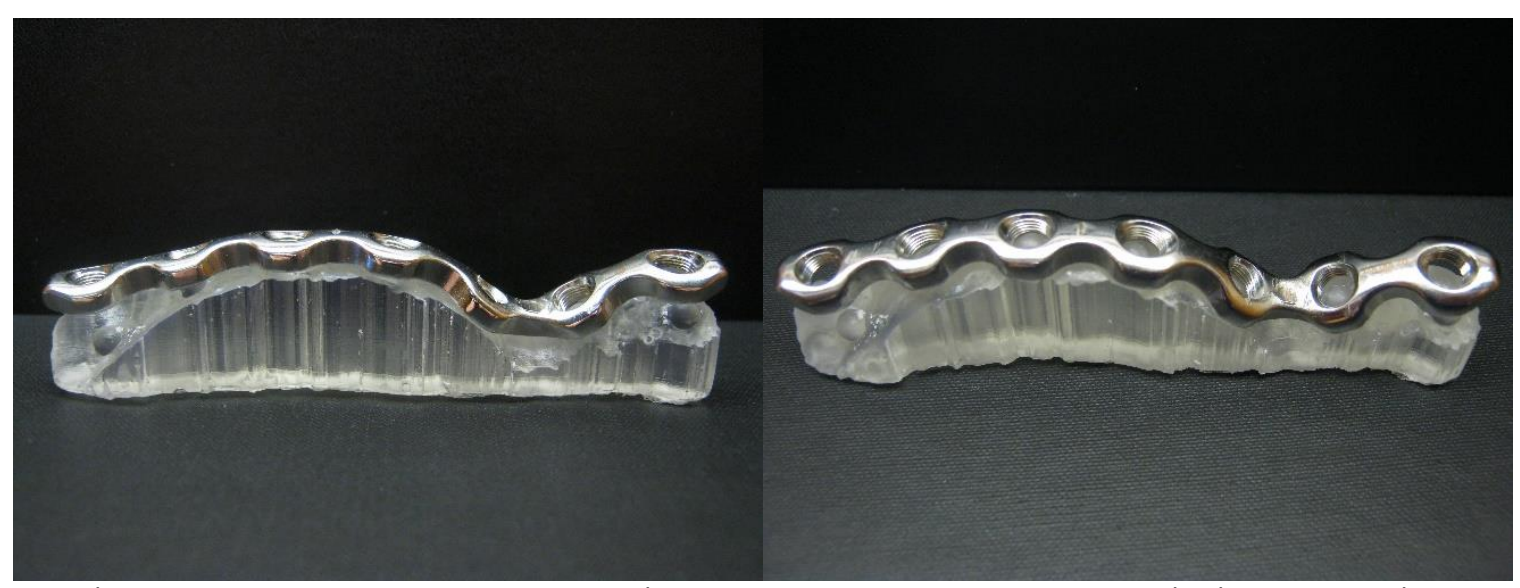

Figure 26 - A Reconstruction Plate Bent to an Initial Bending Template.

The initial template designs revealed several flaws within their construction including the dimensions and structure of the screw landmarks. Other feedback included the desire for the plate to be free standing such as seen in Figure 26. The bent plate shown in Figure 26 does not fully conform to the plate but was an initial bending attempt and not performed by an orthopedic surgeon.

B. Proof of Process and Size Verification

The physical bending template for the posterior wall plate, the reconstruction plate contoured to the templates, and the dimensions of the virtual and physical plate bases and calibration cubes can be seen in Figures 27 and 28 and Table III respectively. 


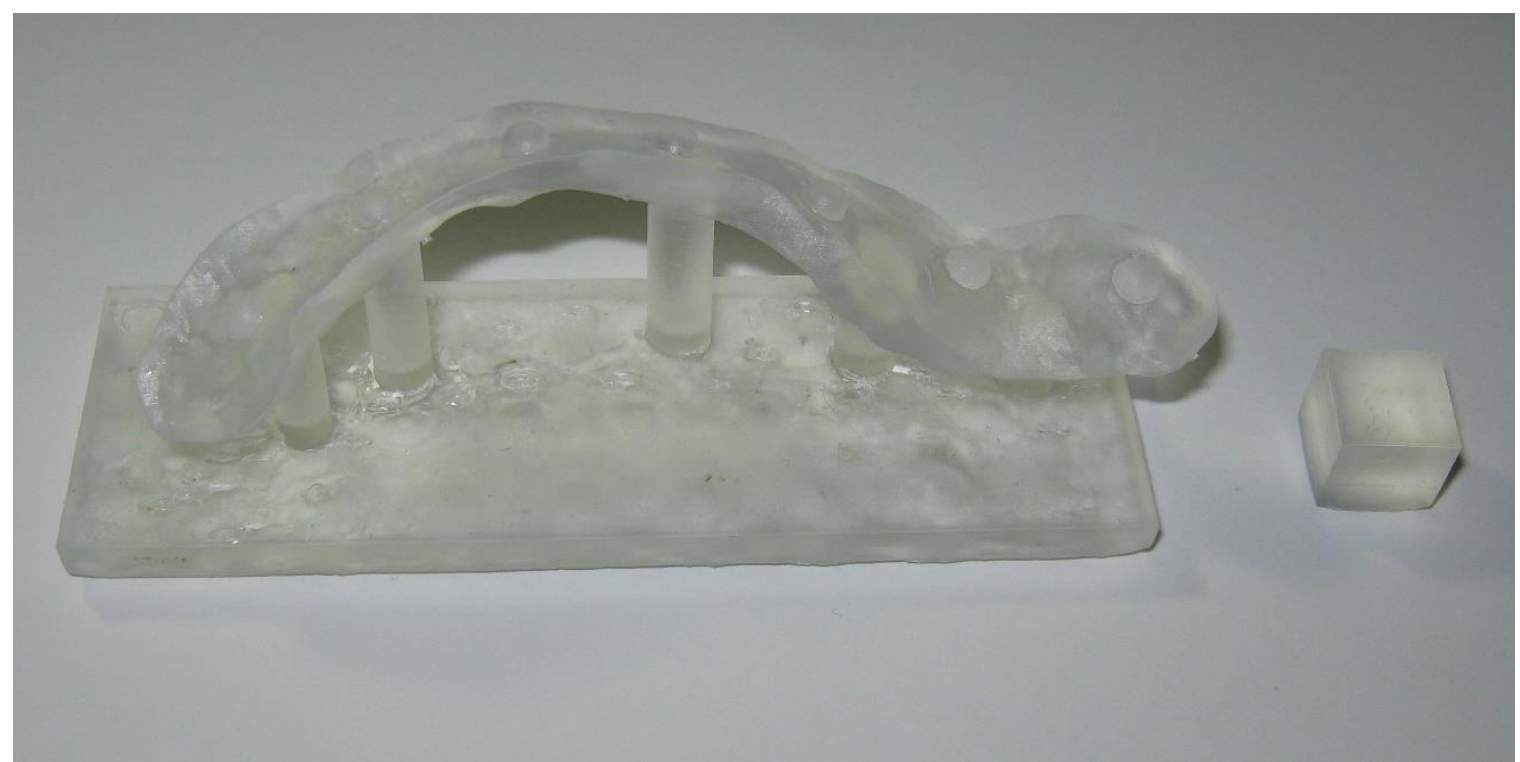

Figure 27 - Proof of Process Bending Template and Isotropic Cube.

Table III - Comparison of Designed and Measured Dimensions.

\begin{tabular}{|c|c|c|c|c|c|c|c|c|}
\hline Part & Direction & Specified $(\mathrm{mm})$ & $\mathrm{m} 1(\mathrm{~mm})$ & $\mathrm{m} 2(\mathrm{~mm})$ & $\mathrm{m} 3(\mathrm{~mm})$ & m_avg $(\mathrm{mm})$ & m_std dev $(\mathrm{mm})$ & \% Error \\
\hline Posterior & $\mathrm{X}$ & 29.00 & 29.17 & 29.18 & 29.09 & 29.1466667 & 0.040 & $0.51 \%$ \\
\cline { 2 - 10 } Wall & $\mathrm{Y}$ & 88.00 & 88.18 & 88.36 & 88.29 & 88.2766667 & 0.074 & $0.31 \%$ \\
\cline { 2 - 9 } Plate & $\mathrm{Z}$ & 4.00 & 5.13 & 4.62 & 4.82 & 4.85666667 & 0.210 & $21.42 \%$ \\
\hline \multirow{2}{*}{$\begin{array}{c}\text { Isotropic } \\
\text { Cube }\end{array}$} & $\mathrm{X}$ & 10.00 & 10.11 & 10.13 & 10.13 & 10.1233333 & 0.009 & $1.23 \%$ \\
\cline { 2 - 9 } & $\mathrm{Y}$ & 10.00 & 10.09 & 10.08 & 10.08 & 10.0833333 & 0.005 & $0.83 \%$ \\
\cline { 2 - 9 } & $\mathrm{Z}$ & 10.00 & 10.19 & 10.25 & 10.17 & 10.2033333 & 0.034 & $2.03 \%$ \\
\hline
\end{tabular}

The plate bending was performed by an orthopedic

surgeon so it can be assumed that the plate was correctly

bent. When examining the dimensions of the printed parts, four of the six measurements fell below 1\% error. The Z, or height, measurement of the cube had over $20 \%$ error. This error is believed to be due to the nature of the uncured polymer present and the density of the support structures. After being printed, the part was washed in IPA to dissolve the uncured liquid resin that is in excess on that part. In 
this case, the quantity of support structures present between the base of the bending template and the printing tray and the very low height created an environment in which the IPA could not adequately flow through to remove the resin. It is probable that some excess resin was still present in this location during the curing process and so this excess resin, instead of being removed from the part as it was in other locations, cured and became joined to the bending template. If we examine the same dimension in the isotropic cube, there is only an error of $2 \%$, showing that this was not a print failure but instead was caused by inadequate post-processing. In the future, placing the base of the bending template directly on the printing platform without support material in between or defining a greater support height may provide a more accurate print with fewer places for uncured resin to remain.

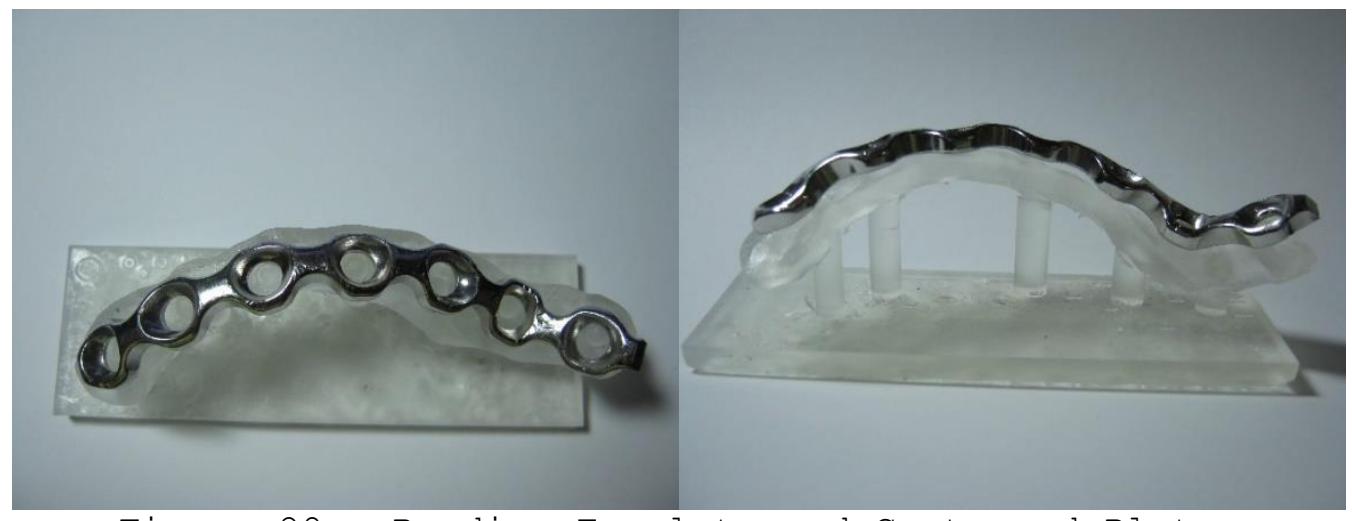

Figure 28 - Bending Template and Contoured Plate. 
C. Segmentation Threshold and Model Size

A total of three segmentations were performed, 31, 37, and 43. All models were built using the $1 \mathrm{~mm}$ and $0.5 \mathrm{~mm}$ slice thickness. Additionally, the threshold 37 model was rebuilt with $3 \mathrm{~mm}$ slices. Table IV shows the relevant comparisons of the models with their average and maximum deviations. Data of all performed comparisons is available in Appendix B. As seen in Table II, the initial comparison of the Threshold 37 model that had $1 \mathrm{~mm}$ and $0.5 \mathrm{~mm}$ slice thickness (named "T37-fc-Before") vs. the Threshold 37 model with manually created $3 \mathrm{~mm}$ slice thickness (named "T37-3mm-Before") had a large maximum distance. Figure 29 shows where these large deviations were physically located on the model. 


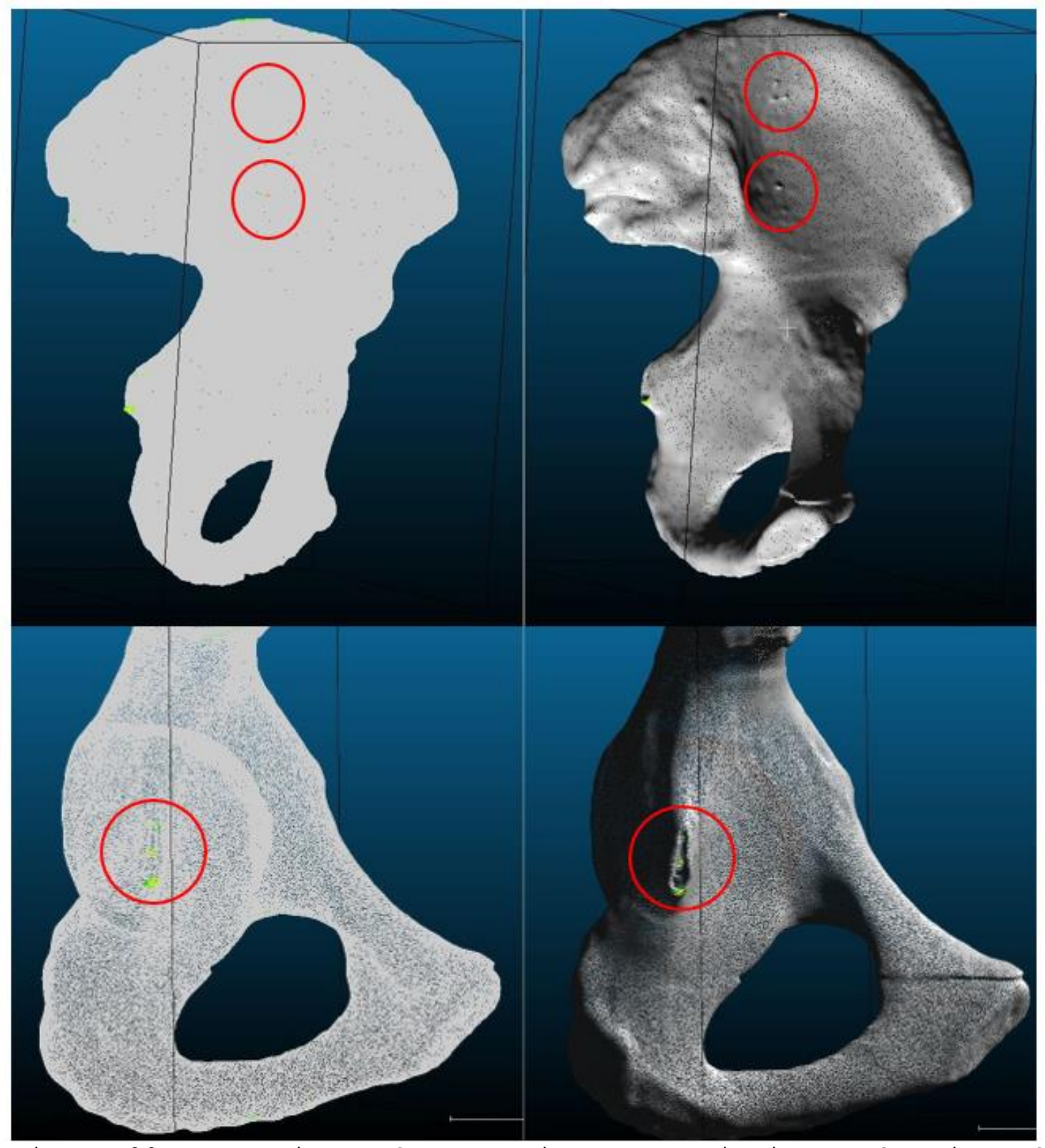

Figure 29 - Locations of Large Distance Deviations. The wing of the ilium (Top) and the ischial spine (Bottom) with distance deviations greater than $1.5 \mathrm{~mm}$ highlighted in red, yellow and green on the left images. The defect in the ischial spine is due to the removal of the sacrospinous ligament and the defect of the ilium wing is most likely the result of soft tissue that wasn't fully removed.

As seen, this error is not due to large differences in the manner of reconstruction but instead due to noise and unfilled sections on the pelvic model. In order to reduce 
the error associated with this noise, manual editing of the images was performed as seen in Figures 30-32, the models were reconstructed (named "T37-fc-After" and "T37-3mmAfter") as seen in Figure 33, and the comparisons were performed again.

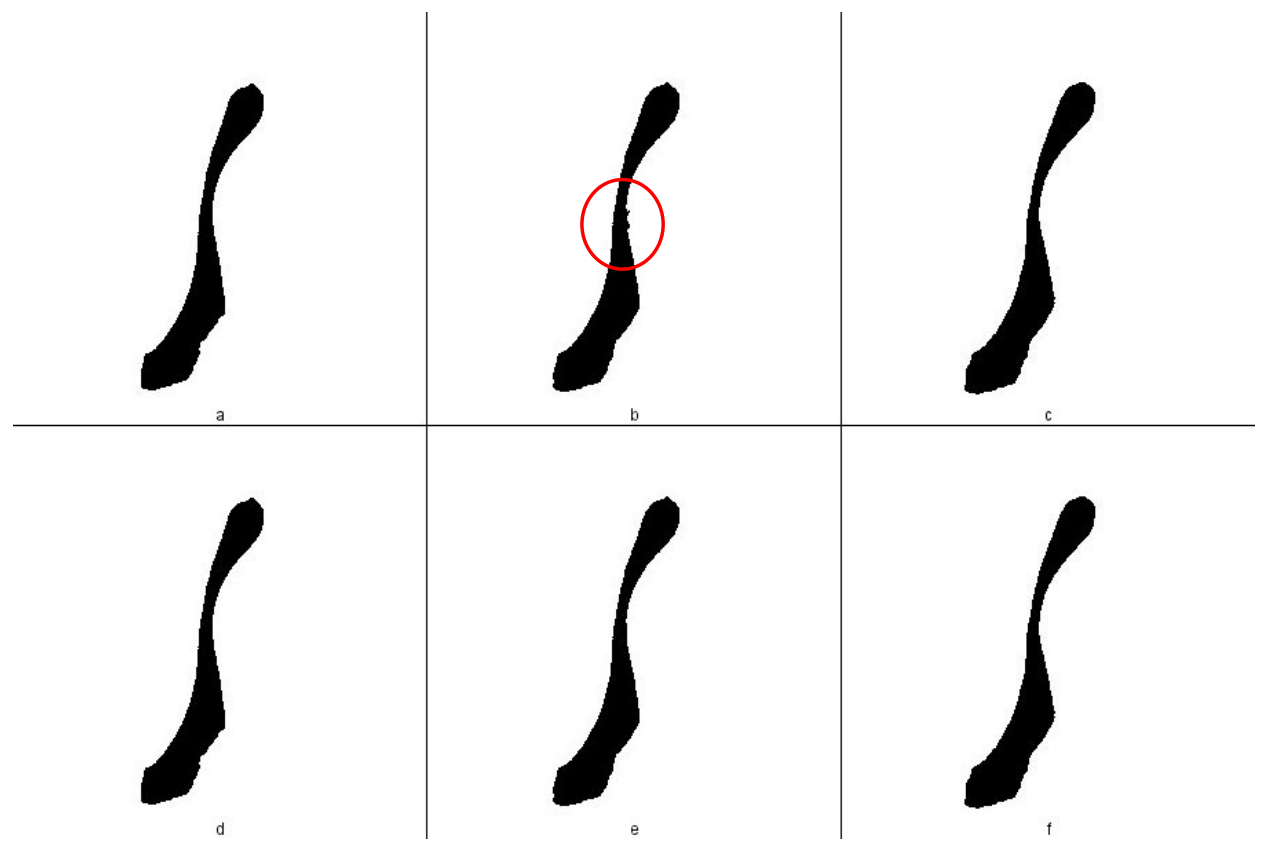

Figure 30 - Repair in T37 Slices 62-65. A-C and D-F show slices 62-65 before and after noise removal respectively. B represents the identical slices 63 and 64 before editing and E represents the identical slices 63 and 64 after the editing. 


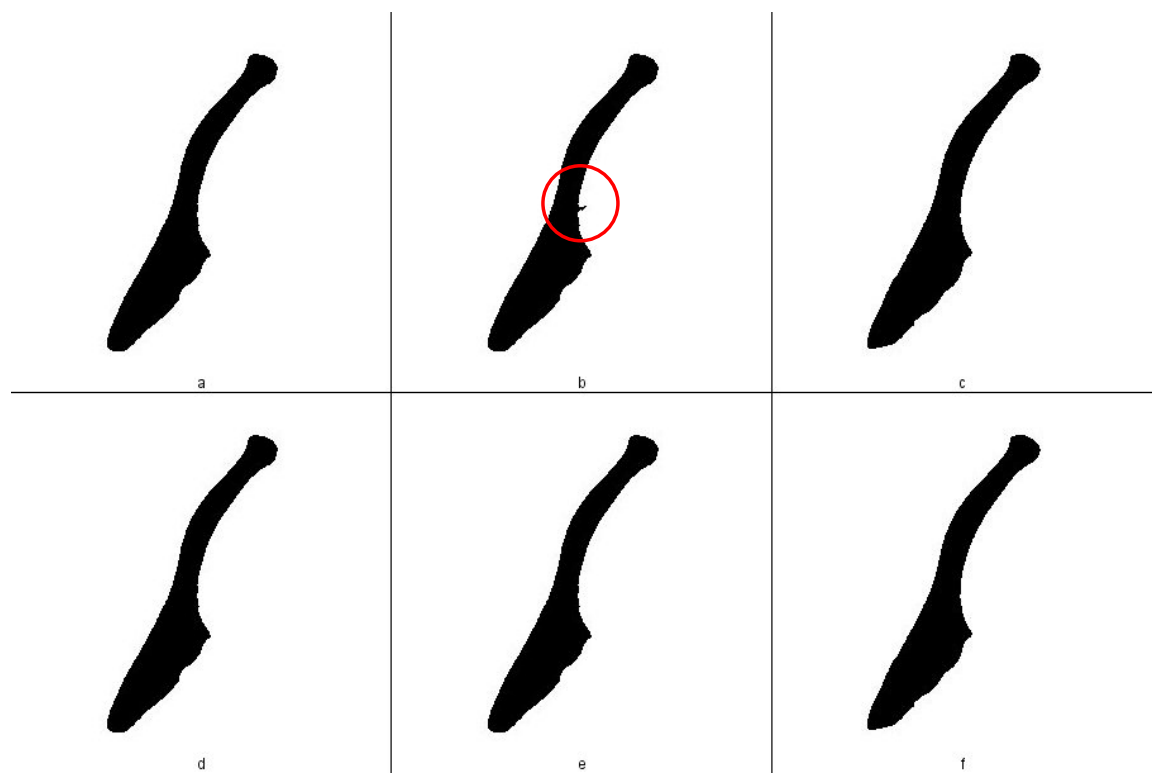

Figure 31 - Repair in T37 Slices 122-125. A-C and D-F show slices 62-65 before and after noise removal respectively. B represent the identical slices 123 and 124 before editing and E represent the identical slices 123 and 124 after the editing.

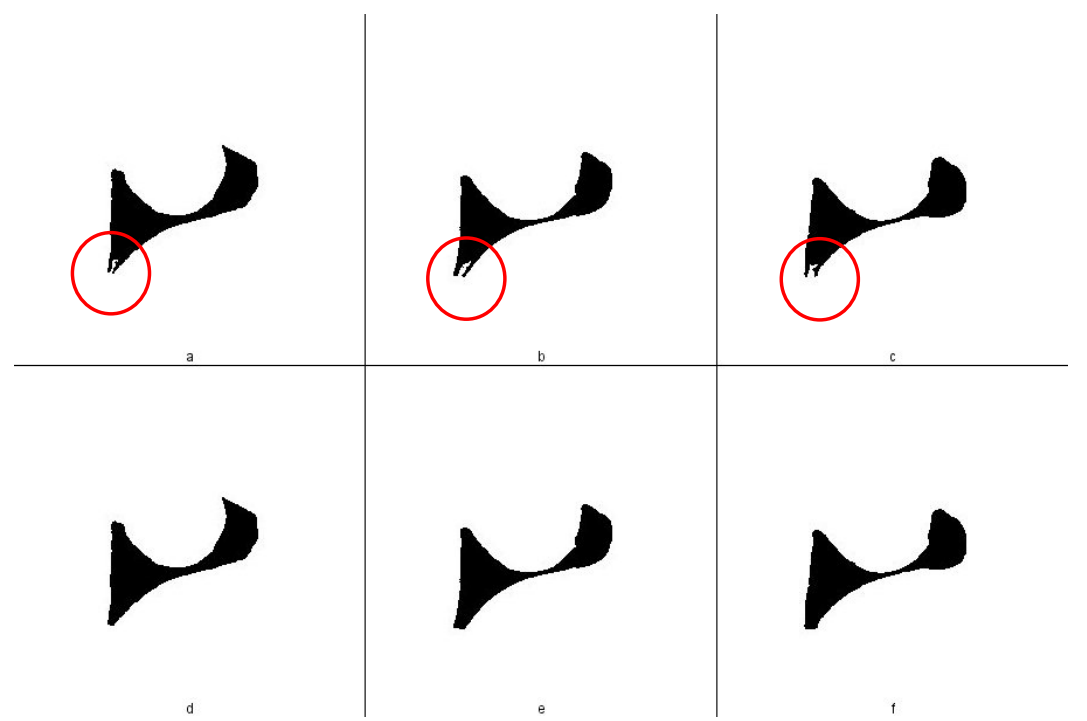

Figure 32 - Repair in T37 Slices $242-264$. A-C are photos of three slices before editing and $\mathrm{D}-\mathrm{C}$ are photos of the same slices after editing. 


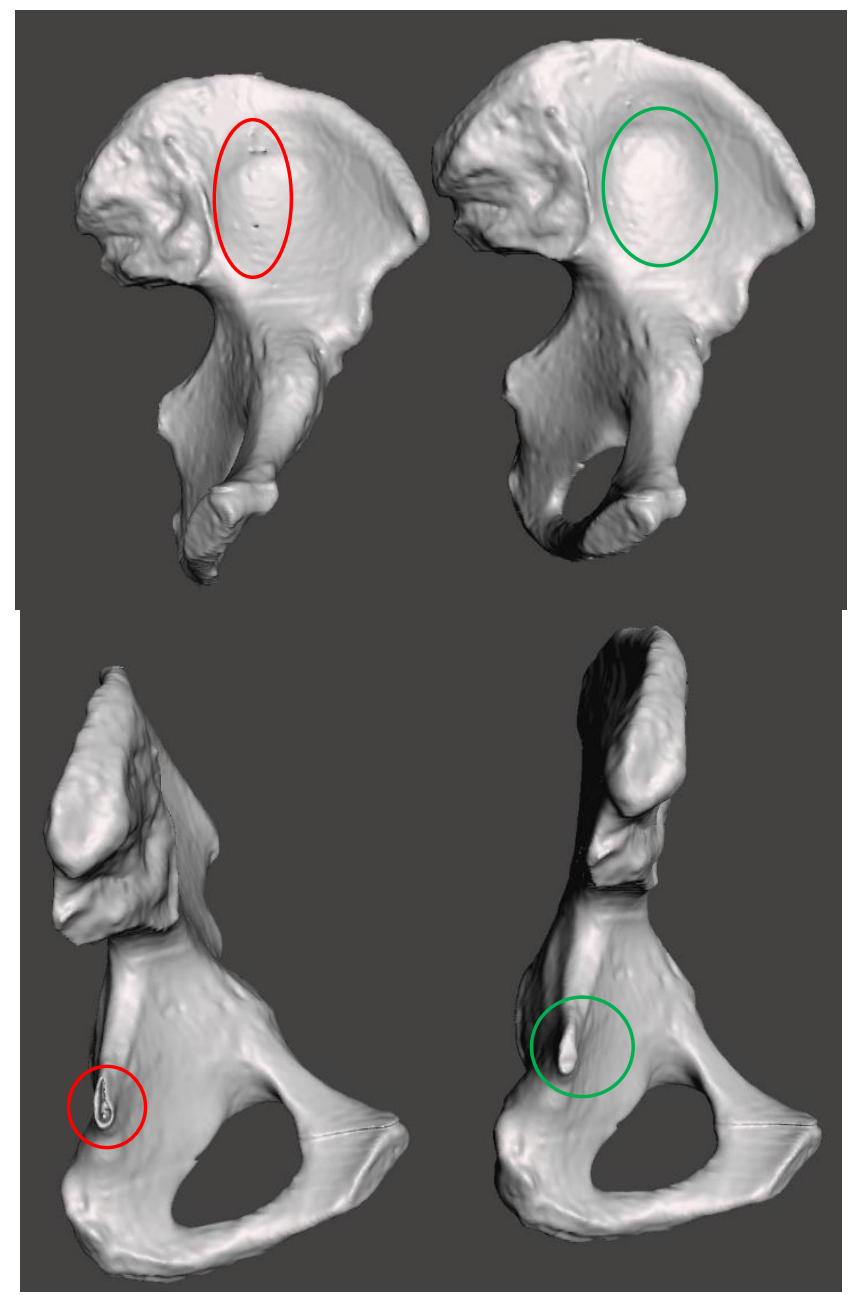

Figure 33 - Before and After Noise Removal. Left-hand images show the model before and right-hand images show the model after noise removal and defect repair.

Table IV - Relevant Distance Values from Model Comparison

\begin{tabular}{|c|c|c|c|c|c|c|c|}
\hline aligned & reference & compared & reference & RMS & $\begin{array}{c}\text { avg } \\
(\mathbf{m m})\end{array}$ & $\begin{array}{c}\text { std dev } \\
(\mathbf{m m})\end{array}$ & $\begin{array}{c}\text { max } \\
(\mathbf{m m})\end{array}$ \\
\hline T37-fc Before & T37-3mm Before & T37-fc Before & T37-3mm Before & 0.5596 & 0.1797 & 0.1358 & 2.5697 \\
\hline T37-3mm After & T37-fc After & T37-fc After & T37-3mm After & 0.5543 & 0.1777 & 0.1289 & 1.3776 \\
\hline T31 & T37-fc-Before & T37-fc-Before & T31 & 0.5271 & 0.1113 & 0.0582 & 1.9958 \\
\hline T43 & T37-fc-Before & T37-fc-Before & T43 & 0.5224 & 0.1087 & 0.0499 & 2.5322 \\
\hline T31 & T43 & T31 & T43 & 0.5335 & 0.1309 & 0.0721 & 2.6379 \\
\hline
\end{tabular}




\title{
V. DISCUSSION
}

\author{
A. Comparison to Current Literature
}

3D Printed models in orthopedic surgery have increased the ability of surgeons to plan for complex surgeries through direct visualization of fracture patterns and advanced personalized medicine by allowing surgeons to pre-bend plates according to the patient's own anatomy. However, all current 3D printed models suffer the severe drawback in the amount of time required to print a fullscale model and, if they are being taken into the operating room, the time required to sterilize them. Methods that involve contouring the plate outside of the operating room before the surgery still suffer from large time delays since the plate must go through a very lengthy sterilization process to be implanted. Additive manufacturing in titanium is the pinnacle of personalized medicine and patient specific plates but the current regulatory environment restricts it from being used 
consistently. Virtual planning software provides even better surgical planning methods than physical models by allowing the surgeon to practice steps of the surgery on the patient's anatomy beforehand. However, most surgical planning software does little to assist the surgeon in the operating room besides the practice provided. Chen et al.'s recent use of MIMICS provides the best combination of surgical planning and physical outputs with the creation of a screw guide and template. Our process allows the surgeon to build the plate, screw by screw, utilizing the existing anatomy and location of fracture lines. As our plate is build, constraints guide the placement of subsequent screws and screw holes. Since our work in intended to be done with locking plates, the orientation of the plate itself guides where the screws will be placed. Additionally, their methods would not have allowed for an accurately dimensioned plate or screw locations if they had performed their process on more complex anatomy than the anterior brim. Their plastic implant was also not manufactured in a way that it would have been feasible to use on a live patient. 
B. Design Choices in the Blender Extension

1. Bending an STL Model of a Plate. One of the initial iterations of the software was designed to function similarly to what Derand et al. described when they deformed an STL model of the reconstruction plate around a virtual model of the mandible [26]. During this iteration, a simple six-hole plate was designed in Solidworks with similar dimensions to a stryker six-hole reconstruction plate. The user would place the 3D cursor on the pelvis, press the 'Import Plate' button on the side panel, the plate would be imported with its midpoint at the 3D cursor location, and would be oriented based on the normal vectors of the surrounding triangles. Along with the plate, a lattice would be created in Blender which would be skinned to the pelvis. The lattice was necessary to get to the plate to accurately contour to the pelvis since several modifiers are available in Blender that allow accurate movements of STL files to be determined by a lattice structure. Once the user imported the lattice file, they would be able to use the left and right direction keys to rotate the plate and lattice together and the plate would contour to the pelvis as it rotated. The size and resolution of the lattice structure could be controlled in 
all three dimensions. Increasing the height dimension often created poor results but increasing the width of the lattice structure enabled the plate to make realistic twisting motions. Increasing the resolution, or number of segments, in the length of the lattice controlled how accurately the out-of-plane bending was performed.

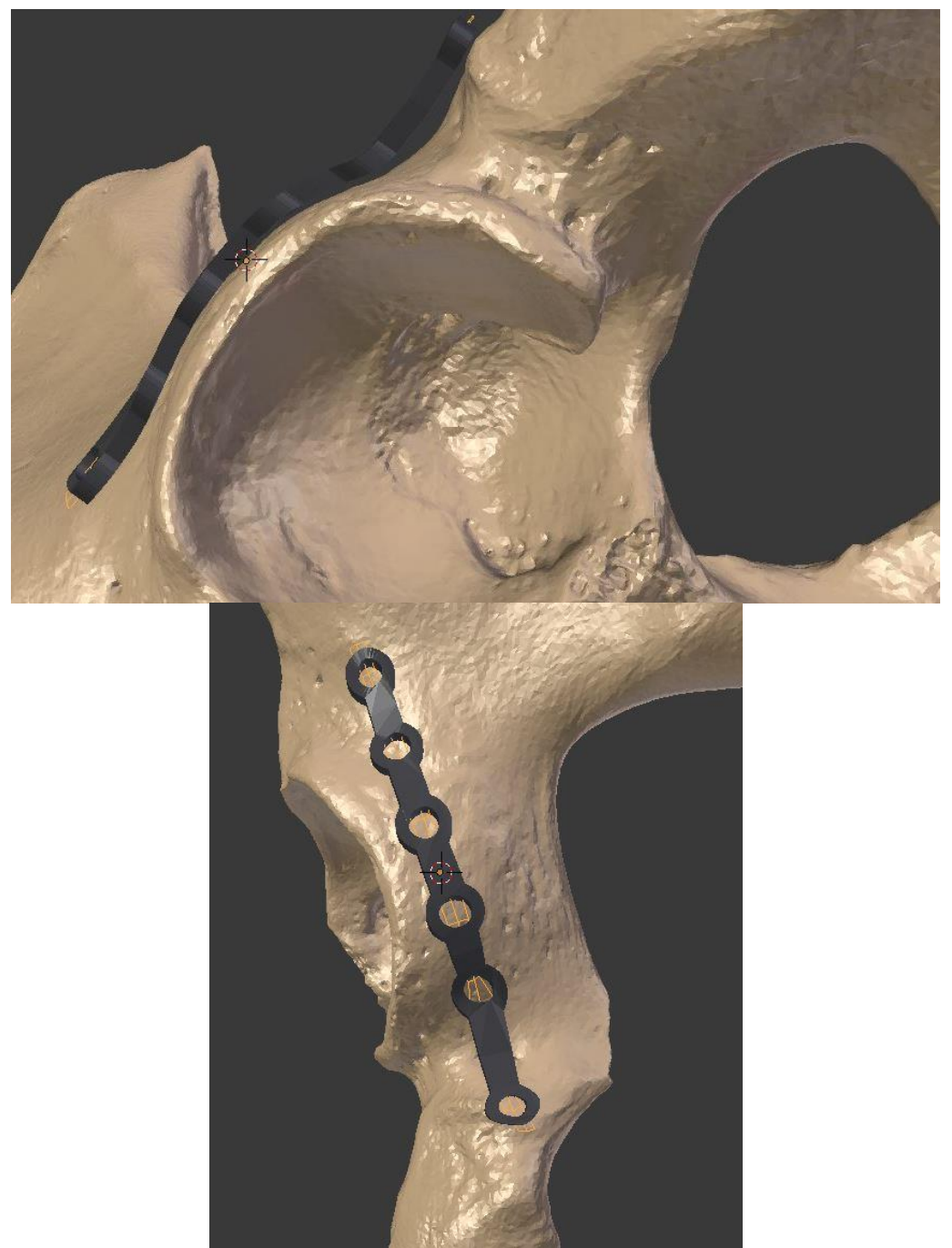

Figure 34 - Normal Bending of STL Model of Plate. Even when the model bend as designed, the dimensions between holes could not be controlled. 
This initial test mimicked the bending shown by Derand to a certain extend but had several key flaws. Firstly, this iteration lacked the ability to undergo out of plane bending without the user manually dragging lattice control points to desired locations. Secondly, dimensional accuracy was not able to be controlled after the plate began being deformed. Thirdly, the lattice method and thin features in the pelvic model combined to cause glitches that could only be fixed by the user manually repositioning the lattice control points.

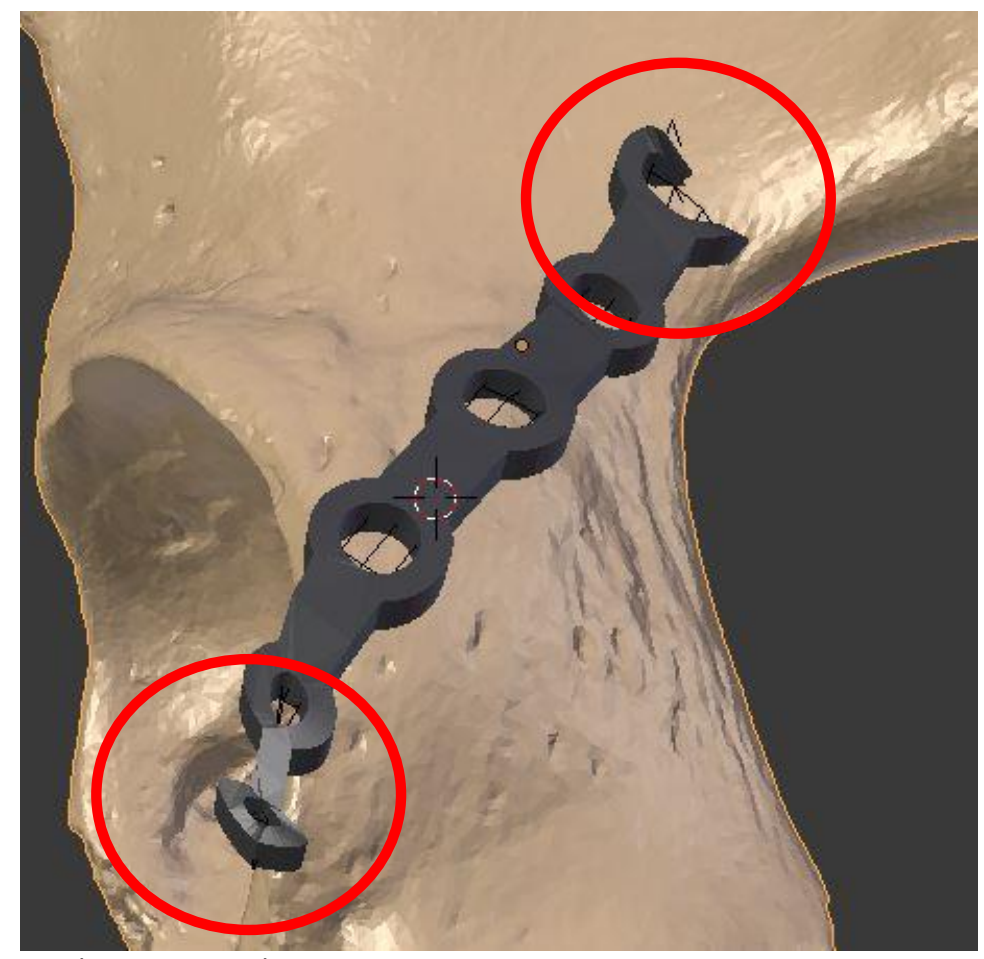

Figure 35 - Glitches with Pre-Made STL Model of Plate. Portions of the plate can be seen 'falling through' the pelvis model or undergoing severe deformations. 
Finally, the method in general did not produce a useable output. Even if the plate had perfect dimensional accuracy, the locations of screws could be changed to allow for bending, and the software glitches were resolved, the only output of this software would have been a contoured, plastic plate. Such a plate would only be useful for its aesthetic value since such high barriers exist to manufacturing a virtual plate in titanium and implanting it in a patient. For these reasons, future work focused on designing a system that would capturing the pelvic surface features while a user built an aesthetic, virtual plate.

\section{Distance Between Screws. To create a usable} bending template, the distance between the screw landmarks had to be controlled in a way that would mimic a physical plate. Chen's study focused on a geometrically simple location, the anterior brim of the pelvis, and dimensioned their screws to be $12 \mathrm{~mm}$ apart from one another. Initially, the software proposed in this work used a similar method by defining the center of each screw hole as a point and constraining those points to be $13 \mathrm{~mm}$ from one another. 


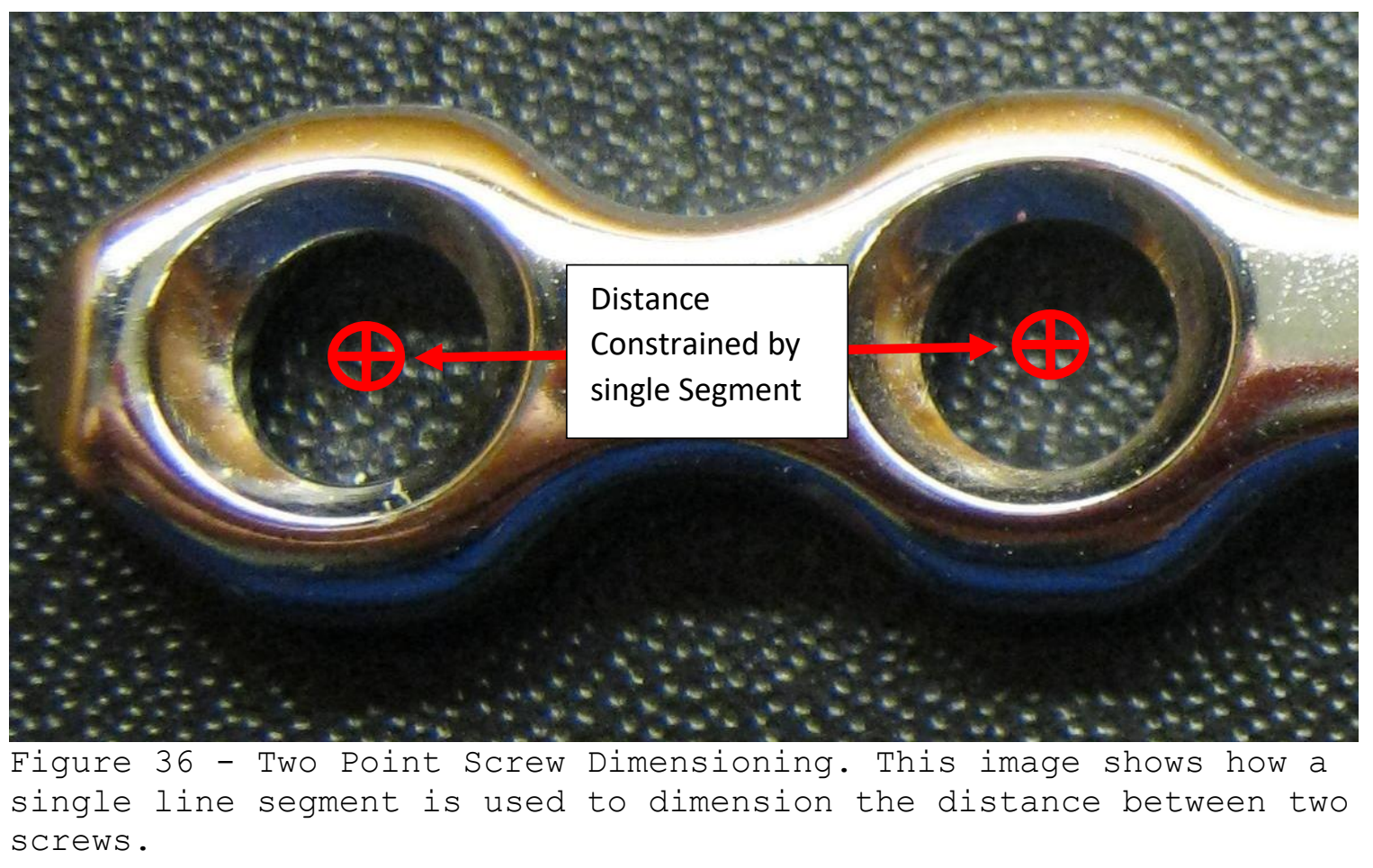

This method will give perfect results on a flat surface and adequate results on surfaces with mild curves. As the complexity of the surface increases however, only using two points to constrain the distance between screws will provide increasingly inaccurate results. To overcome this, our work incorporated five points with four straight line segments for dimensioning. There is no real limit to how many points can be included but we felt that diminishing returns would be seen after a more than five were included.

3. Screw Landmarks. Creating landmarks to show the locations of screws is an important component of this work. Initial designs were shown earlier and were less than 
desirable for a variety of reasons. The screw holes in our plates were $4.5 \mathrm{~mm}$ in diameter so the initial landmarks were designed to be cylinders with a $4.4 \mathrm{~mm}$ diameter. The intention was that the user would bend the reconstruction plate so that it would sit perfectly on the template surface and all cylinders would be fully seated in the screw hole locations as seen in Figure 37.

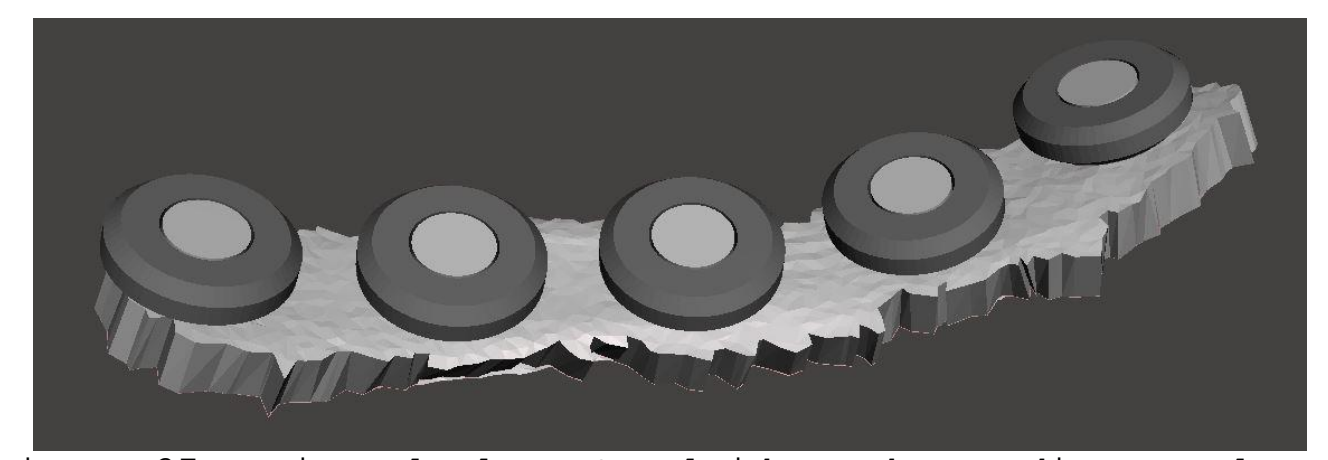

Figure 37 - Virtual Plate Overlaid on the Bending Template.

This method proved to be problematic once a physical bending template was created and physical reconstruction plates were countered to it. Firstly, the cylinders fit inside the screw holes of the plate as intended while the plate was flat but after bending, even with the holes protected, small dimension changes made the cylinders unable to fit into the screw holes. Secondly, sharp bends in the template couple with the size of the cylinders resulted in the plate not being able to be placed on the template due to physical interactions between the two. 
Future attempts included using spheres that were less dimensionally restrictive and allowed the surgeon flexibility in the angle of their plate. The initial testing with these spheres remedied the problems of physical interference between the plate and the template but did not provide the necessary tactile response when a plate had been fully conformed. A final iteration of the screw landmark that was not manufactured was a cylinder that was $2.5 \mathrm{~mm}$ in diameter to allow some amount of flexibility with plate placement. This design was not used due to surgeon preference for the larger spheres.

\section{Future Additions to Software and Process}

1. Additional Blender Features. The software extension developed for Blender functions well in its current state but further improvements could still be made to it. Currently, the software works very well at allowing for full control of screw/plate location while still keeping the plate dimensionally accurate. A downside of this flexibility is that the template created will require for more bending and twisting of the plate to create a perfect fit than a surgeon would ordinarily perform. The final form of the plate will always be determined by the 
physician but certain additions to the software could make the bending process easier. Since many reconstruction plates come with no prior bends, the software should take this into account and, instead of having small degrees of out-of-plane bending between each hole, the software could instead attempt to keep the plate as straight as possible and create fewer out-of-plane bends with larger angles. Other limits to control the amount of twisting or in-plane bending that the plate goes through would also be advantageous to keep the bending realistic with the material properties of the plate.

In the software's current iteration, screw landmarks are placed at every location that a screw hole would be present on the plate. In a surgery however, many of these holes are often unused. Allowing the user to disable the placement of screw landmarks where they knew they would not need screws would allow the user to focus the plate bending on the crucial locations were screws would be placed.

Finally, this software currently accounts for fracture lines by the user manually painting them on the surface of the model. In its current state, this is still a lengthy process and future iterations would add easy methods to quickly draw on a fracture line or indicate where they would like the plate to be placed. As discussed before, a 
computer assisted automatic reduction would all the user to not only know where the fracture lines were when making the model but the divide between two bones would also create a noticeable crease when the bending template is printed. With a physical representation of the fracture line location included with the model, the surgeon would be able to more accurately position their plate in the predetermined location.

\section{Virtual Fragment Reduction. One of the most} personalized surgical planning solutions was shown by Cimerman and Kristan [34] in which the individual bone fragments were separated so that the fracture could be virtually reduced. Other authors have proposed similar systems for mandible [42], pelvic fracture [43], [44], and long bone [45], [46] repair. This work initially included a similar method that involved manual segmentation and reduction of all fracture fragment. Similar to the methods currently used in this work to separate the intact hemipelvis from the rest of an image stack, manual editing and erosions could be used to separate fracture fragments. The MATLAB software described earlier was originally intended to separate multiple fragments of interest and many of its features were designed for the purpose of 
fragment extraction. After being separated and rebuilt as STL models, the fragments could be imported into Blender and the translation and rotation options available within the software would allow the fragments to be reduced. Figure 38 shows an image a pelvis that had its fragments separated and reduced within Blender.

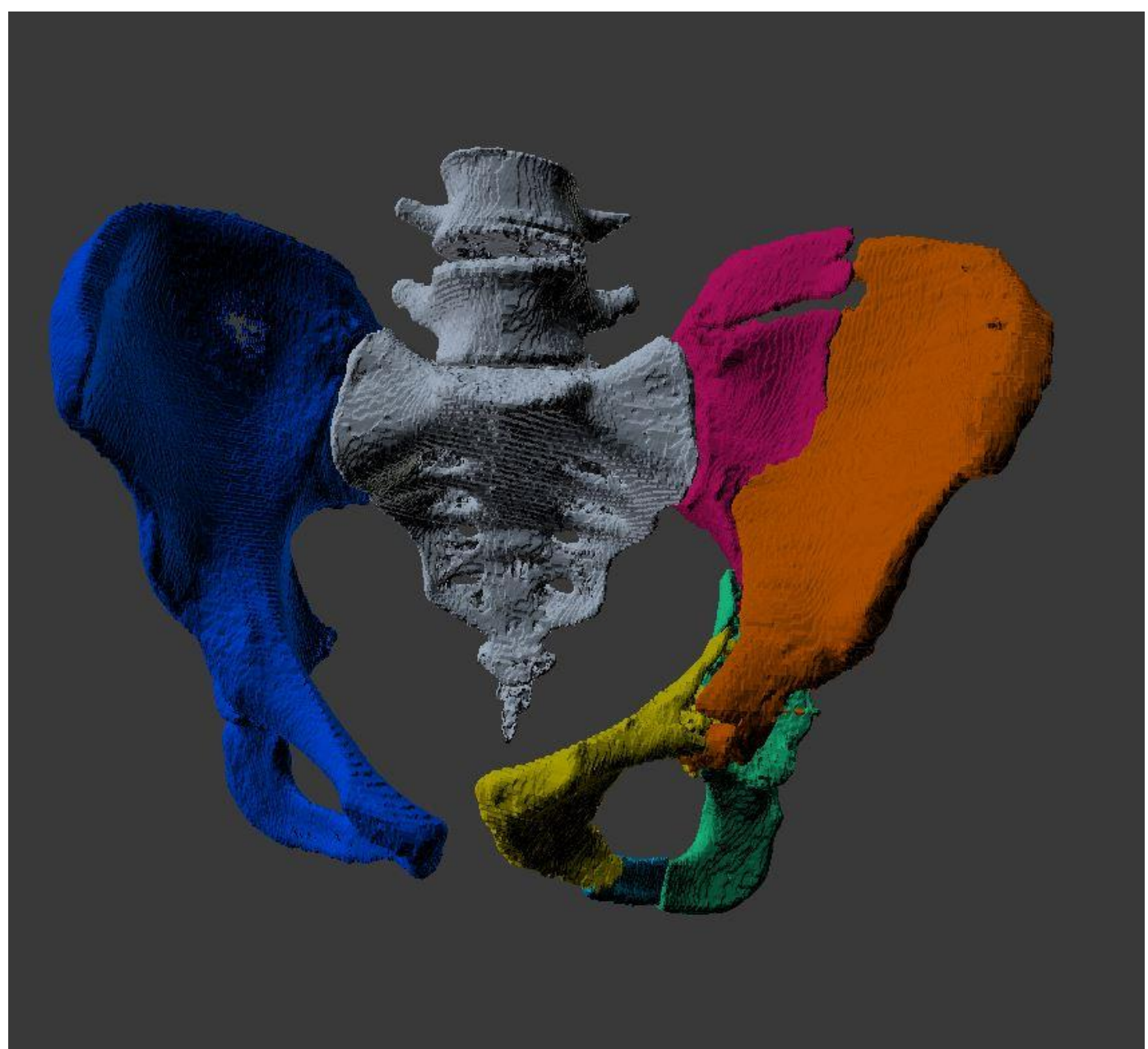

Figure 38 - Reducing Fracture Fragments in Blender.

This method was highly dependent on having a high-quality CT image stack with thin slice thickness to accurately 
separate the bones. The standard slice thickness for orthopedic trauma situations is $3 \mathrm{~mm}$ and the system was tested using $1 \mathrm{~mm}$ slices. Slices thinner than $3 \mathrm{~mm}$ are rarely needed to diagnose fractures and the increased radiation dosage to achieve the required resolution would not be in the patient's best interest. Even with thin sliced images, it is difficult to adequately segment the images and separate the fragments without removing portions of the bone and the manual fragment reduction method is time consuming and not intuitive. An extension with Slicer, called Virtual Fragment Reconstruction, allows the user to input stacks of fracture fragments and a stack of the unfractured bone. Using this information, the software will automatically realign fragments in a maximization of mutual information method. This extension was unavailable during the development of this work but, in the future, could provide an easy method for automated reduction if a thin slice CT is performed.

3. Machine Assisted Plate Bending. Spinal fusion surgeries require metal rods to be bent to hold the patient's vertebrae in the proper orientation while they heal. A 1999 paper described how computers could be used to calculate the bends that spinal rods would need to make to 
accurately conform to the patient and how that information could be transferred to a machine to perform the precise bending needed [47]. The process of contouring of a reconstruction plate is similar to the process of bending a spinal rod and a similar, machine controlled, method could be used to prevent the surgeon from needing to manually bend the plate. This would make the plates more accurate since it would eliminate human error and stronger since constant bending and readjustment of a plate will weaken it and can eventually cause it to fail [48].

\section{Segmentation Threshold and Model Size. Derand [26]}

and Shen et al [33] performed comparisons of bending variability between surgeons, nurses and technicians of both mandibular and pelvic reconstruction plates. Derand found that there was 1.2-1.3mm mean difference between mandibular plates bent by surgeons and Shen et al showed median differences of 0.26-0.80mm between pelvic reconstruction plates bent by surgeons, nurses and technicians. For this work, differences of over $1.0 \mathrm{~mm}$ between models were considered significant. Although all maximum distances presented in Table IV were above the 1.0mm threshold, almost all of those maximum differences were at either the noise/defect locations already shown in 
Figure 29, at the very top of the iliac wing as seen in Figure 40, at the very bottom at the ischial tuberosity, or at other miscellaneous overhang locations as seen in Figure 41. The only significant location of deviation that came close to the $1.0 \mathrm{~mm}$ threshold in all models was between "T37-fC-After" and "T37-3mm-After" at a location were an anterior brim or pubic symphysis plate would be placed. The distance was approximately $0.9 \mathrm{~mm}$ and is shown in Figure 39. Otherwise, no significant deviations occurred at locations where anterior brim, ilioinguinal, posterior column, or posterior wall plates would be placed.

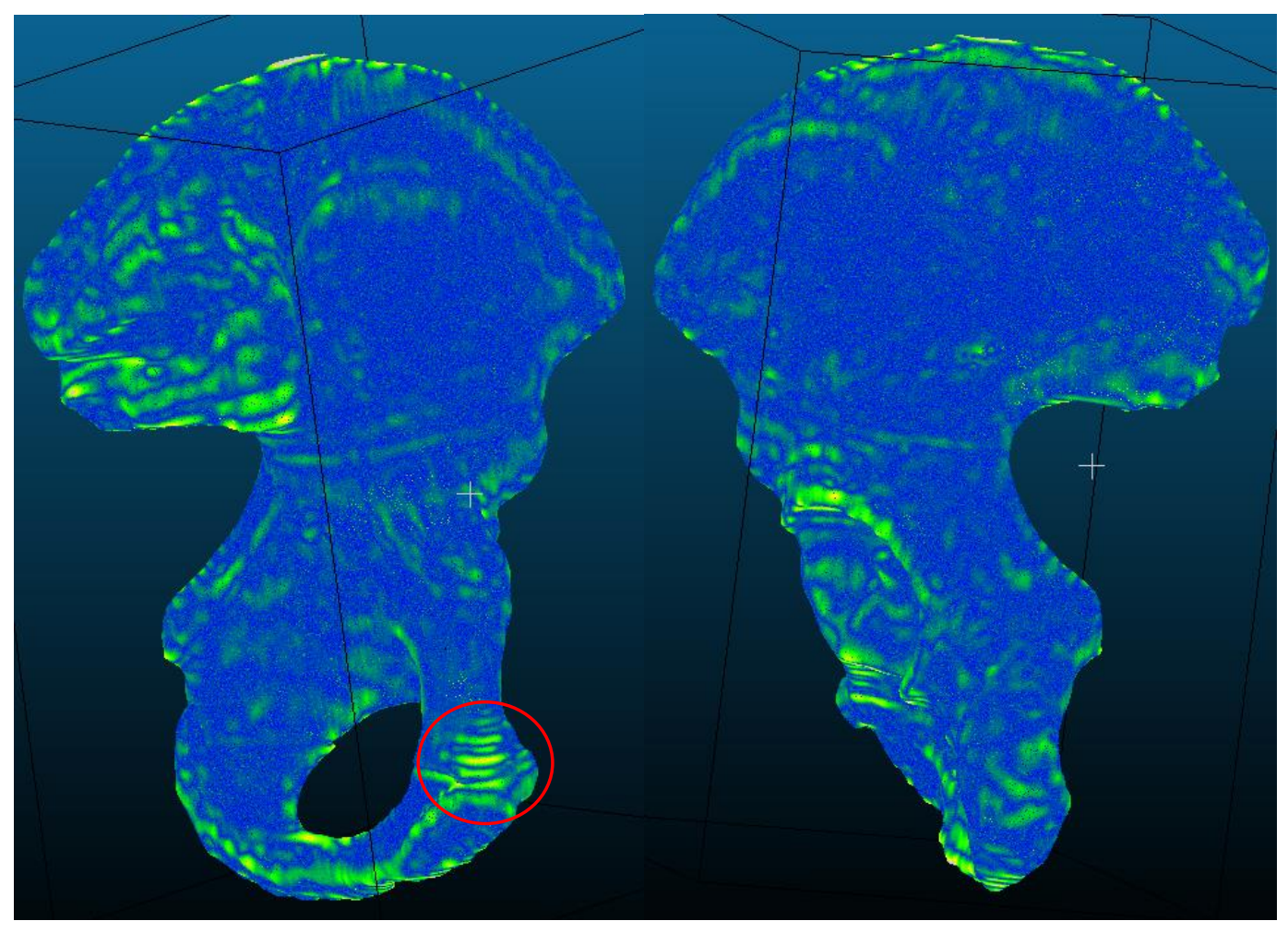

Figure 39 - Maximum Deviations at Plate Locations. Showing the front (Left) and back (right) of the iliac wing. 


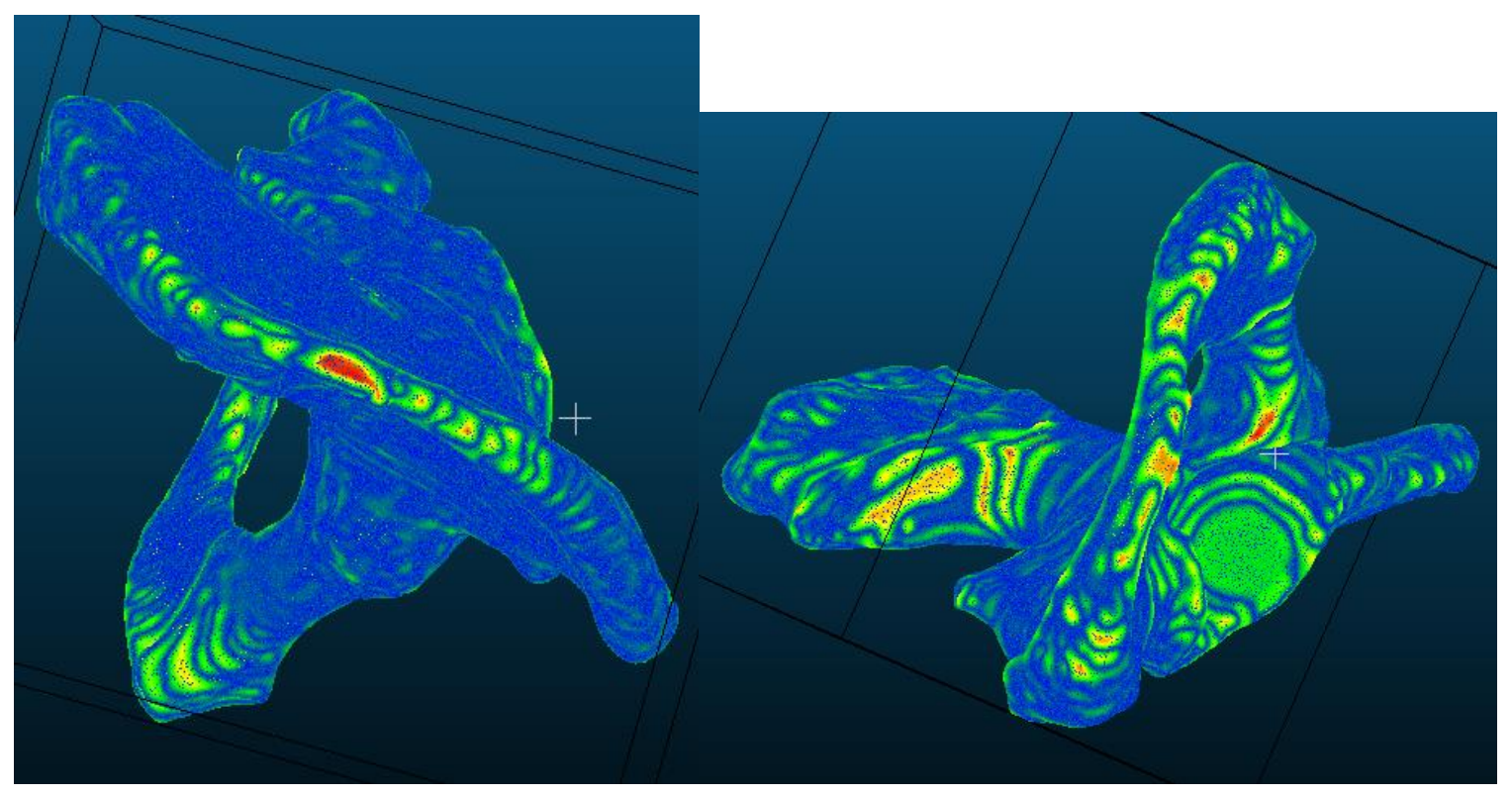

Figure 40 - Maximum Deviations at Insignificant Locations. Deviations larges than $1 \mathrm{~mm}$ at the very top of the iliac wing (left) and overhangs and the bottom of the ischial tuberosity (right).

\section{E. Patient Study}

It was not possible to incorporate a patient study into this thesis work however, using a patient's CT data to design their reconstruction plate is the logical next step. Most scans taken during pelvic trauma are $3 \mathrm{~mm}$ in thickness. This work has shown that no significant deviations exist between models constructed with very thin slices, $0.5-$ 1.0mm, and those constructed from 3mm slices. Additionally, the orthopedic surgeon will always have the final decision in how a plate is bent before it is implanted in the patient. Sterilization was mentioned in this work as a step 
in the process but was not able to be performed for the proof of process. However, minimal logistic or financial barriers exist to printing the plate in an autoclavable material and flash sterilizing it in the oR suite's autoclave before it is used in the operating room. Recorded outcomes of the surgery would be the operative time, blood lost by the patient, time required by the surgeon to bend the plate, additional time required to create the template, the quality of the final reduction, and a qualitative analysis of how helpful or burdensome the creation and use of a bending template was for the surgery. As the patient returns for follow up, infection, other adverse events, and how well the plate has maintained the reduction should be recorded. While nothing could be statistically proven with a single patient case, this study would provide the ground work to design further study that could statistically prove that the use of a patient specific bending template provides better outcomes to the patient. 


\section{CONCLUSION}

This thesis has shown that the patient specific pre-bending of plates is an accessible and low-cost solution to ensuring patients with orthopedic pelvic injuries receive the best possible treatment. Additionally, work by other authors has suggested that using pre-bent plates can reduce operative time, blood loss, and allow for less invasive procedures. Future work should focus on the optimization of software, automatic reconstruction of bone fragments, streamlining the process to reduce burden on the physician, and proving that the process can be performed for a patient in a timely manner. Further studies should focus on proving that the use of a patient specific bending template results in shorter operative times, less blood loss, and fewer adverse events to the patient. Further work around virtual reduction and automated machine bending will further increase the accuracy and specificity of reconstruction plate bending and reduce the time needed to accurately contour them. 


\section{APPENDIX}

\section{A. Text Output from MATLAB During Proof of Process}

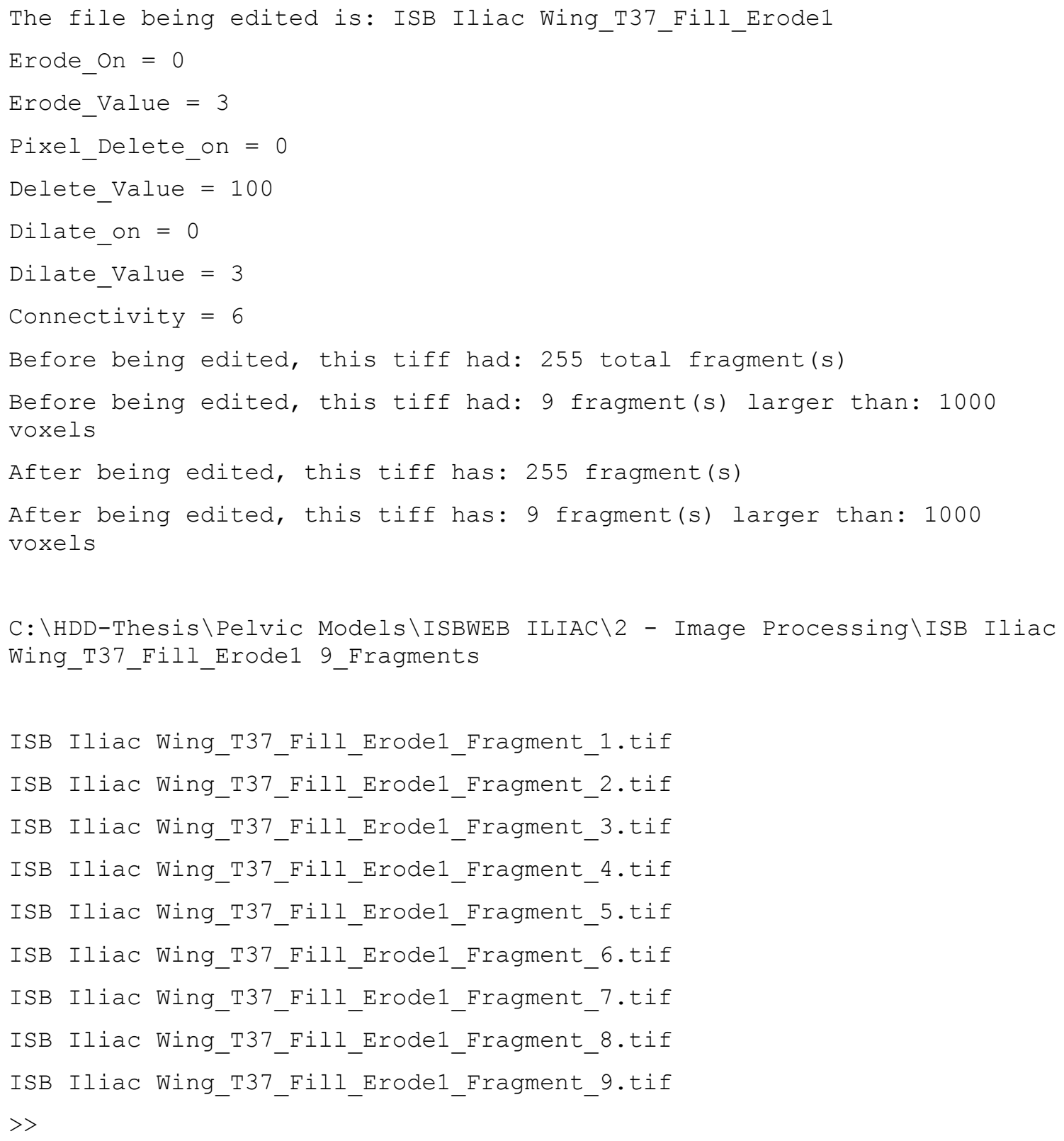




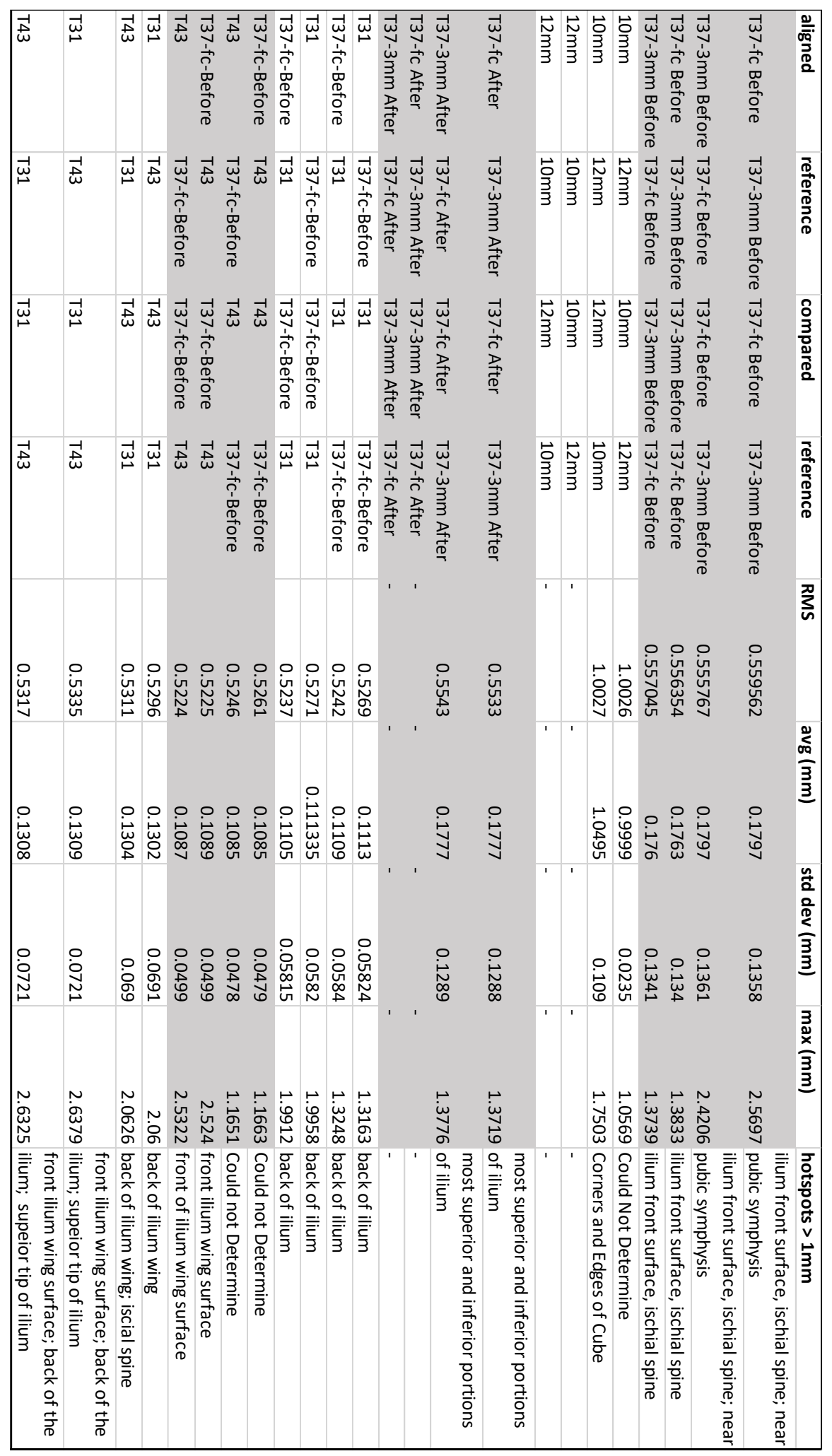




\section{LIST OF REFERENCES}

[1] M. S. Taljanovic, M. D. Jones, J. T. Ruth, J. B. Benjamin, J. E. Sheppard, and T. B. Hunter, "Fracture Fixation," RadioGraphics, vol. 23, no. 6, pp. 15691590, Nov. 2003.

[2] A. K. Obaid, A. Barleben, D. Porral, S. Lush, and M. Cinat, "Utility of Plain Film Pelvic Radiographs in Blunt Trauma Patients in the Emergency Department," Am. Surg., vol. 72, no. 10, pp. 951-954, 2006.

[3] K. Lunsjo, A. Tadros, A. Hauggaard, R. Blomgren, J. Kopke, and F. Abu-Zidan, "Acute plain anterioposterior radiograph of the pelvis is not useful in detecting fractures of iliac wing and os sacrum: A prospective study of 73 patients using CT as gold standard," Australas. Radiol., vol. 51, no. 2, pp. 147-149, Apr. 2007 .

[4] F. S. Shivji, C. Quah, and D. P. Forward, "Pelvic fractures," Surg., vol. 33, no. 6, pp. 257-263, 2015.

[5] R. P. Dunbar and J. A. Lowe, "Pelvic Fractures," 2016. [Online]. Available:

http: / / orthoinfo.aaos.org/topic.cfm?topic=a00520. [Accessed: 08-Jun-2017].

[6] M. Tile, J. Kellam, and D. Helfet, Fractures of the Pelvis and Acetabulum 3rd Ed. LWW, 2003.

[7] J. E. Bible and H. R. Mir, "External Fixation," J. Am. Acad. Orthop. Surg., vol. 23, no. 11, pp. 683-690, Nov. 2015 .

[8] J. Lei, H. Liu, Z. Li, Z. Wang, X. Liu, and L. Zhao, "Biomechanical comparison of fixation systems in posterior wall fracture of acetabular by finite element analysis," Comput. Assist. Surg., vol. 21, no. 1, pp. $117-126,2016$.

[9] R. Dimitriou and P. V. Giannoudis, "Pelvic fractures," Surg., vol. 30, no. 7, pp. 339-346, 2012.

[10] P. Gillespie, "Pelvic fracture," Surgery, vol. 27, no. 7, pp. 292-296, 2009.

[11] P. L. Rice and M. Rudolph, "Pelvic Fractures," Emerg. Med. Clin. North Am., vol. 25, no. 3, pp. 795-802, 2007 .

[12] R. JUDET, J. JUDET, and E. LETOURNEL, "Fractures of the Acetabulum: Classification and Surgical Approaches for Open Reduction. Preliminary Report.," J. Bone Joint Surg. Am., vol. 46, no. 4, pp. 1615-46, 1964. 
[13] Acumed, "Acumed Pelvic Plating System," 2016. [Online] Available: http://www.acumed.net/. [Accessed: 14 June 2017]

[14] Q. Liu, K. Zhang, Y. Zhuang, K. Tao, and Y. Fu, "A morphological study of anatomical plates for acetabular posterior column," Int. J. Comput. Assist. Radiol. Surg., vol. 9, no. 4, pp. 725-731, 2014.

[15] Stryker, "Matta Pelvic System: Pelvic and Acetabular Plating," 2001. [Online]. Available:

http://www.stryker.com/. [Accessed: 14 June 2017]

[16] Depuysynthes, "Pelvic Implants and Instruments. A dedicated system for reconstructive pelvic and acetabular surgery.," 2016. [Online] Available: http://www.depuysynthes.com. [Accessed: 14 June 2017]

[17] J.-H. Hwang, J.-K. Oh, C.-W. Oh, Y.-C. Yoon, and H. W. Choi, "Mismatch of anatomically pre-shaped locking plate on asian femurs could lead to malalignment in the minimally invasive plating of distal femoral fractures: a cadaveric study," Arch. Orthop. Trauma Surg., vol. 132, no. 1, pp. 51-56, Jan. 2012.

[18] M. Gardner and M. B. Henley, Illustrated Tips and Tricks in Fracture Surgery. LWW, 2010.

[19] A. Stannard, A. Schmidt, and P. Kregor, Surgical Treatment of Orthopedic Trauma. Thieme Medical Publishers, Inc., 2007.

[20] P. V. Giannoudis, C. Tzioupis, A. Papathanassopoulos, O. Obakponovwe, and C. Roberts, "Articular step-off and risk of post-traumatic osteoarthritis. Evidence today," Injury, vol. 41, no. 10, pp. 986-995, 2010.

[21] M. Frame and J. S. Huntley, "Rapid Prototyping in Orthopaedic Surgery: A User's Guide," Sci. World J., vol. 2012, pp. 1-7, 2012.

[22] J. M. Duncan, S. Nahas, K. Akhtar, and J. Daurka, "The Use of a 3D Printer in Pre-operative Planning for a Patient Requiring Acetabular Reconstructive Surgery.," J. Orthop. case reports, vol. 5, no. 1, pp. 23-5, 2015.

[23] G. A. Brown, B. Milner, and K. Firoozbakhsh, "Application of computer-generated stereolithography and interpositioning template in acetabular fractures: a report of eight cases.," J. Orthop. Trauma, vol. 16, no. 5, pp. 347-52, 2002 .

[24] T. Niikura et al., "Tactile surgical navigation system for complex acetabular fracture surgery," Orthopedics, vol. 37, no. 4. pp. 237-242, 2014.

[25] C. Rahimov and I. Farzaliyev, "Virtual bending of titanium reconstructive plates for mandibular defect bridging: review of three clinical cases.," 
Craniomaxillofac. Trauma Reconstr., vol. 4, no. 4, pp. 223-34, Dec. 2011.

[26] P. Dérand and J.-M. Hirsch, "Virtual Bending of Mandibular Reconstruction Plates Using a Computer-Aided Design," J. Oral Maxillofac. Surg., vol. 67, no. 8, pp. $1640-1643,2009$.

[27] A. Cohen, A. Laviv, P. Berman, R. Nashef, and J. AbuTair, "Mandibular reconstruction using stereolithographic 3-dimensional printing modeling technology," Oral Surgery, Oral Med. Oral Pathol. Oral Radiol. Endodontology, vol. 108, no. 5, pp. 661-666, 2009.

[28] M. I. Salgueiro and M. R. Stevens, "Experience with the use of prebent plates for the reconstruction of mandibular defects.," Craniomaxillofac. Trauma Reconstr., vol. 3, no. 4, pp. 201-8, Dec. 2010.

[29] D. Wang et al., "Customized a Ti6Al4V bone plate for complex pelvic fracture by selective laser melting," Materials (Basel)., vol. 10, no. 1, pp. 1-14, 2017.

[30] L. Yan, Y. Yuan, L. Ouyang, H. Li, A. Mirzasadeghi, and L. Li, "Improved mechanical properties of the new Ti15Ta-xzr alloys fabricated by selective laser melting for biomedical application," J. Alloys Compd., vol. 688, pp. 156-162, 2016.

[31] P. Liu et al., "A study on the mechanical characteristics of the EBM-printed Ti-6Al-4V LCP plates in vitro," J. Orthop. Surg. Res., vol. 9, no. 1, p. 106, Dec. 2014.

[32] F. S. L. Bobbert et al., "Additively manufactured metallic porous biomaterials based on minimal surfaces: A unique combination of topological, mechanical, and mass transport properties," Acta Biomater., vol. 53, pp. 572-584, 2017.

[33] F. Shen, B. Chen, Q. Guo, Y. Qi, and Y. Shen, "Augmented reality patient-specific reconstruction plate design for pelvic and acetabular fracture surgery," Int. J. Comput. Assist. Radiol. Surg., vol. 8, no. 2, pp. 169-179, 2013.

[34] M. Cimerman and A. Kristan, "Preoperative planning in pelvic and acetabular surgery: The value of advanced computerised planning modules," Injury, vol. 38, no. 4, pp. 442-449, 2007 .

[35] X. Chen et al., "Accurate fixation of plates and screws for the treatment of acetabular fractures using 3Dprinted guiding templates: An experimental study," Injury, vol. 48, no. 6, pp. 1147-1154, 2017.

[36] W. Chen, Z. Hou, Y. Su, W. R. Smith, F. A. Liporace, 
and Y. Zhang, "Treatment of posterior pelvic ring disruptions using a minimally invasive adjustable plate," Injury, vol. 44, no. 7, pp. 975-980, 2013.

[37] C. A. Schneider, W. S. Rasband, and K. W. Eliceiri, "NIH Image to ImageJ: 25 years of image analysis," Nat. Methods, vol. 9, no. 7, 2012.

[38] A. Fedorov et al., "3D Slicer as an image computing platform for the Quantitative Imaging Network.," Magn. Reson. Imaging, vol. 30, no. 9, pp. 1323-41, Nov. 2012.

[39] Formlabs, "Form 2 Desktop SLA Printer." [Online]. Available: https://formlabs.com/3d-printers/form-2/. [Accessed: 07-Jun-2016].

[40] Formlabs, "3D Printing Technology Comparison: SLA vs. DLP," 2016. [Online]. Available:

https://formlabs.com/blog/3d-printing-technologycomparison-sla-dlp/. [Accessed: 07-Jun-2017].

[41] D. Rickets, "High Resolution Human Female Pelvis Scan by dricketts - Thingiverse." [Online]. Available: https://www.thingiverse.com/thing:1645117. [Accessed: 01-Aug-2016].

[42] J. O. Voss, V. Varjas, J. D. Raguse, N. Thieme, R. G. Richards, and L. Kamer, "Computed tomography-based virtual fracture reduction techniques in bimandibular fractures," J. Cranio-Maxillofacial Surg., vol. 44, no. 2 , pp. 177-185, 2016.

[43] M. Citak et al., "Virtual 3D planning of acetabular fracture reduction," J. Orthop. Res., vol. 26, no. 4, pp. 547-552, 2008 .

[44] M. Krcah, G. Szekely, and R. Blanc, "Fully Automatic and Fast Segmentation of the Femur Bone from 3D-CT Imagez with No Shape Prior," in IEEE International Symposium on Biomedical Imaging: From Nano to Macro, 2011.

[45] D. M. Kahler, "Navigated Long-Bone Fracture Reduction," J. Bone Jt. Surgery-American Vol., vol. 91, no. Suppl 1 , pp. 102-107, 2009.

[46] Y. Chen, M. Qiang, K. Zhang, H. Li, and D. Hao, "Novel Computer-Assisted Preoperative Planning System for Humeral Shaft Fractures: Report of 43 Cases," Int. J. Med. Robot., vol. 11, pp. 109-119, 2015.

[47] B. Paizer et al., "A Pilot Study on Computer-Assisted Optimal Contouring of Orthopedic Fixation Devices," vol. 4, pp. 305-313, 1999.

[48] B. Gervais, A. Vadean, M. Raison, and M. Brochu, "Failure analysis of a $316 \mathrm{~L}$ stainless steel femoral orthopedic implant," 2016. 


\title{
IX. VITA
}

\author{
Gordon Lents \\ Benton, KY 42025 \\ gordon.lents@gmail.com
}

\section{EDUCATION :}

Doctor of Medicine August 2017 - May 2021 (Expected)

University of Kentucky, College of Medicine

Lexington, KY

Master of Engineering May 2016 - August 2017

Bioengineering

University of Louisville, J. B. Speed School of Engineering Louisville, KY

Bachelor of Science $\quad$ August 2012 - May 2016

Bioengineering

University of Louisville, J. B. Speed School of Engineering Louisville, KY

\section{WORK EXPERIENCE :}

Graduate Teaching Assistant August 2016 - December 2016 Engineering Methods, Tools, and Practice I

University of Louisville

Louisville, KY

Engineering Coop January 2016 - May 2016 Otolaryngology Head and Neck Surgery

Cook Medical

Bloomington, IN

\section{Engineering Coop}

Corrective and Preventive Action

Cook Medical

Bloomington, IN

Engineering Coop

August 2014 - December 2014

Peripheral Intervention 
Cook Medical

Bloomington, IN

First Year Guide

August 2013 - September 2013

University of Louisville

Louisville, KY

REACH Ambassador

University of Louisville

August 2013 - May 2014

Louisville, KY

\section{VOLUNTEER EXPERIENCE:}

Emergency Room Volunteer

May 2015 - May 2016

University of Louisville Hospital

Louisville, KY

Laboratory Volunteer

August 2016 - December 2016

Cardiovascular Innovations Institute

Dr. George Pantalos

Louisville, KY

Laboratory Volunteer

January 2015 - May 2016

Orthopedic Bioengineering Laboratory

Dr. Michael Voor

Louisville, KY

Tutor

Cochran Elementary

Louisville, KY

\section{HONORS AND AWARDS:}

Bioengineering Alumni Award

Trustee's Scholarship

August 2012 - May 2017

\section{ORGANI ZATIONS :}

Tau Beta Pi

Pi Kappa Alpha Fraternity
October 2015 - Present

August 2013 - May 2016 